\title{
INTERSECTION THEORY IN DIFFERENTIAL ALGEBRAIC GEOMETRY: GENERIC INTERSECTIONS AND THE DIFFERENTIAL CHOW FORM
}

\author{
XIAO-SHAN GAO, WEI LI, AND CHUN-MING YUAN
}

\begin{abstract}
In this paper, an intersection theory for generic differential polynomials is presented. The intersection of an irreducible differential variety of dimension $d$ and order $h$ with a generic differential hypersurface of order $s$ is shown to be an irreducible variety of dimension $d-1$ and order $h+s$. As a consequence, the dimension conjecture for generic differential polynomials is proved. Based on intersection theory, the Chow form for an irreducible differential variety is defined and most of the properties of the Chow form in the algebraic case are established for its differential counterpart. Furthermore, the generalized differential Chow form is defined and its properties are proved. As an application of the generalized differential Chow form, the differential resultant of $n+1$ generic differential polynomials in $n$ variables is defined and properties similar to that of the Macaulay resultant for multivariate polynomials are proved.
\end{abstract}

\section{Contents}

1. Introduction

2. Preliminaries

2.1. Differential polynomial algebra and Kolchin topology

2.2. Characteristic sets of a differential polynomial set

2.3. Dimension and order of a prime differential polynomial ideal

2.4. A property on differential specialization

3. Intersection theory for generic differential polynomials

3.1. Generic dimension theorem

3.2. Order of a system of generic differential polynomials

4. Chow form for an irreducible differential variety

4.1. Definition of the differential Chow form

4.2. The order of the differential Chow form

4.3. Differential Chow form is differentially homogenous

4.4. Factorization of the differential Chow form

4.5. Leading differential degree of an irreducible differential variety

4.6. Relations between the differential Chow form and the variety

5. Differential Chow variety

Received by the editors August 19, 2010 and, in revised form, May 7, 2011.

2010 Mathematics Subject Classification. Primary 12H05, 14C05; Secondary 14C17, 14Q99.

Key words and phrases. Differential Chow form, differential Chow variety, differential resultant, dimension conjecture, intersection theory, differential algebraic cycle, differential Stickelberger's Theorem, generic differential polynomial.

This work was partially supported by a National Key Basic Research Project of China (2011CB302400) and by a grant from NSFC (60821002). 
5.1. Sufficient conditions for a polynomial to be

a differential Chow form

5.2. Differential Chow quasi-variety for a differential algebraic cycle

6. Generalized differential Chow form and differential resultant

6.1. Generalized differential Chow form

6.2. Differential resultant of multivariate differential polynomials

7. Conclusion

References

\section{INTRODUCTION}

Differential algebra or differential algebraic geometry founded by Ritt and Kolchin aims to study algebraic differential equations in a similar way in which polynomial equations are studied in commutative algebra or algebraic geometry [32, 19. Therefore, the basic concepts of commutative differential algebra are based on those of commutative algebra. An excellent survey on this subject can be found in [4.

It is known that, for many properties in algebraic geometry, their differential counterparts are much more difficult to prove and some of them are still open problems. For instance, many of the 16 questions proposed by Ritt in his classic book Differential Algebra [32, p.177] are still not solved. In this paper, two naturally connected problems in differential algebraic geometry are studied: the differential dimension conjecture for generic differential polynomials and the differential Chow form.

The first part of the paper is concerned with the differential dimension conjecture which is one of the problems proposed by Ritt: Let $F_{1}, \ldots, F_{r}$ be differential polynomials in $\mathcal{F}\left\{y_{1}, \ldots, y_{n}\right\}$ with $r<n$, where $\mathcal{F}$ is a differential field. If the differential variety of the system $\left\{F_{1}, \ldots, F_{r}\right\}$ is nonempty, then each of its components is of dimension at least $n-r$ [32, p.178].

Ritt proved that the conjecture is correct when $r=1$, that is, any component of a differential polynomial equation in $\mathcal{F}\left\{y_{1}, \ldots, y_{n}\right\}$ is of dimension $n-1$ [32, p.57]. The general differential dimension conjecture is still open. In 8 , it is shown that the differential dimension conjecture is closely related with Jacobi's bound for the order of differential polynomial systems, which is another well-known conjecture in differential algebra.

In this paper, we consider the dimension and order for the intersection of an irreducible differential variety with generic differential hypersurfaces. A differential polynomial $f$ is said to be generic of order $s$ and degree $m$ if $f$ contains all the monomials with degree less than or equal to $m$ in $y_{1}, \ldots, y_{n}$ and their derivatives of order up to $s$, and the coefficients of $f$ are differential indeterminates. A generic differential hypersurface is the set of solutions of a generic differential polynomial. We show that for generic differential hypersurfaces, we can determine the dimension and order of their intersection with an irreducible differential variety explicitly. More precisely, we will prove

Theorem 1.1. Let $\mathcal{I}$ be a prime differential polynomial ideal in $\mathcal{F}\left\{y_{1}, \ldots, y_{n}\right\}$ with dimension $d$ and order $h$ and $f$ a generic differential polynomial with order $s$ and degree greater than zero. If $d>0$, then $\mathcal{I}_{1}=[\mathcal{I}, f]$ is a prime differential polynomial 
ideal in $\mathcal{F}\left\langle\mathbf{u}_{f}\right\rangle\left\{y_{1}, \ldots, y_{n}\right\}$ with dimension $d-1$ and order $h+s$, where $\mathbf{u}_{f}$ is the set of coefficients of $f$. Also, if $d=0, \mathcal{I}_{1}$ is the unit ideal in $\mathcal{F}\left\langle\mathbf{u}_{f}\right\rangle\left\{y_{1}, \ldots, y_{n}\right\}$.

As a direct consequence of this result, we show that the dimension conjecture is valid for a system of generic differential polynomials. Furthermore, the order of the system is also given explicitly.

Another purpose for studying the intersection of an irreducible differential variety with generic differential hypersurfaces is to establish the theory of the differential Chow form, which is the concern of the second part of the paper consisting of Sections 4 to 6 .

The Chow form, also known as the Cayley form or the Cayley-Chow form, is a basic concept in algebraic geometry [41, 16]. More recently, the Chow form also became a powerful tool in elimination theory. This is not surprising, since the Chow form is a resultant in a certain sense. The Chow form was used as a tool to obtain deep results in transcendental number theory by Nesterenko [27] and Philippon 29. Brownawell made a major breakthrough in elimination theory by developing new properties of the Chow form and proving an effective version of the Nullstellensatz with optimal bounds [3. Gel'fand et al. and Sturmfels started the sparse elimination theory, which is to study the Chow form and the resultant associated with sparse polynomials [13, 40]. Eisenbud et al. proposed a new expression for the Chow form via exterior algebra and used it to give explicit formulas in many new cases [11. Jeronimo et al. gave a bounded probabilistic algorithm which can be used to compute the Chow form, whose complexity is polynomial in the size and the geometric degree of the input equation system [17. Other properties of the Chow form can be found in [28, 30, 38]. Given the fact that the Chow form plays an important role in both theoretic and algorithmic aspects of algebraic geometry and has applications in many fields, it is worthwhile to develop the theory of the differential Chow form and hope that it will play a similar role as its algebraic counterpart.

Let $V$ be an irreducible differential variety of dimension $d$ in an $n$-dimensional differential affine space and

$$
\mathbb{P}_{i}=u_{i 0}+u_{i 1} y_{1}+\cdots+u_{i n} y_{n}(i=0, \ldots, d)
$$

$d+1$ generic hyperplanes in differential variables $y_{1}, \ldots, y_{n}$, where $u_{i j}(i=0, \ldots, d$; $j=0, \ldots, n)$ are differential indeterminates. The differential Chow form of $V$ is roughly defined to be the elimination differential polynomial in $u_{i j}$ for the intersection of $V$ with $\mathbb{P}_{i}=0(i=0, \ldots, d)$. More intuitively, the differential Chow form of $V$ can be roughly considered as the condition on the coefficients of $\mathbb{P}_{i}$ such that these $d+1$ hyperplanes will meet $V$. We will show that most of the properties of the Chow form in the algebraic case presented in [16, 41] can be generalized to the differential case. Precisely, we will prove

Theorem 1.2. Let $V$ be an irreducible differential variety with dimensiond and order $h$ over a differential field $\mathcal{F}$ and $F\left(\mathbf{u}_{0}, \mathbf{u}_{1}, \ldots, \mathbf{u}_{d}\right) \in \mathcal{F}\left\{\mathbf{u}_{0}, \mathbf{u}_{1}, \ldots, \mathbf{u}_{d}\right\}$ the Chow form of $V$ where $\mathbf{u}_{i}=\left(u_{i 0}, u_{i 1}, \ldots, u_{i n}\right)(i=0,1, \ldots, d)$. Then $F\left(\mathbf{u}_{0}, \mathbf{u}_{1}, \ldots, \mathbf{u}_{d}\right)$ has the following properties:

1. $F\left(\mathbf{u}_{0}, \mathbf{u}_{1}, \ldots, \mathbf{u}_{d}\right)$ is differentially homogenous of the same degree in each set $\mathbf{u}_{i}$ and $\operatorname{ord}\left(F, u_{i j}\right)=h$ for all $u_{i j}$ occurring in $F$. 
2. $F\left(\mathbf{u}_{0}, \mathbf{u}_{1}, \ldots, \mathbf{u}_{d}\right)$ can be factored uniquely into the following form:

$$
\begin{aligned}
F\left(\mathbf{u}_{0}, \mathbf{u}_{1}, \ldots, \mathbf{u}_{d}\right) & =A\left(\mathbf{u}_{0}, \mathbf{u}_{1}, \ldots, \mathbf{u}_{d}\right) \prod_{\tau=1}^{g}\left(u_{00}^{(h)}+\sum_{\rho=1}^{n} u_{0 \rho}^{(h)} \xi_{\tau \rho}+t_{\tau}\right) \\
& =A\left(\mathbf{u}_{0}, \mathbf{u}_{1}, \ldots, \mathbf{u}_{d}\right) \prod_{\tau=1}^{g}\left(u_{00}+\sum_{\rho=1}^{n} u_{0 \rho} \xi_{\tau \rho}\right)^{(h)}
\end{aligned}
$$

where $g=\operatorname{deg}\left(F, u_{00}^{(h)}\right), \xi_{\tau \rho}$ are in a differential extension field $\mathcal{F}_{\tau}$ of $\mathcal{F}$, and $\left(u_{00}+\right.$ $\left.\sum_{\rho=1}^{n} u_{0 \rho} \xi_{\tau \rho}\right)^{(h)}$ is the $h$-th derivative of $\left(u_{00}+\sum_{\rho=1}^{n} u_{0 \rho} \xi_{\tau \rho}\right)$. The first " $="$ is obtained by factoring $F\left(\mathbf{u}_{0}, \mathbf{u}_{1}, \ldots, \mathbf{u}_{d}\right)$ as an algebraic polynomial in the variables $u_{00}^{(h)}, u_{01}^{(h)}, \ldots, u_{0 n}^{(h)}$, while the second " $="$ is a differential expression to be explained in Section 4.4 of this paper.

3. $\Xi_{\tau}=\left(\xi_{\tau 1}, \ldots, \xi_{\tau n}\right)(\tau=1, \ldots, g)$ are generic points of $V$. Also, they are the only elements of $V$ lying on the differential hyperplanes $\mathbb{P}_{\sigma}=0(\sigma=1, \ldots, d)$ as well as on the algebraic hyperplanes ${ }^{a} \mathbb{P}_{0}^{(l)}=0(l=0, \ldots, h-1)$.

4. Suppose that $\mathbf{u}_{i}(i=0, \ldots, d)$ are differentially specialized to sets $\mathbf{v}_{i}$ of specific elements in $\mathcal{F}$ and $\overline{\mathbb{P}}_{i}(i=0, \ldots, d)$ are obtained by substituting $\mathbf{u}_{i}$ by $\mathbf{v}_{i}$ in $\mathbb{P}_{i}$. If $\overline{\mathbb{P}}_{i}=0(i=0, \ldots, d)$ meet $V$, then $F\left(\mathbf{v}_{0}, \ldots, \mathbf{v}_{d}\right)=0$. Furthermore, if $F\left(\mathbf{v}_{0}, \ldots, \mathbf{v}_{d}\right)=0$ and $S_{F}\left(\mathbf{v}_{0}, \ldots, \mathbf{v}_{d}\right) \neq 0$, then the $d+1$ hyperplanes $\overline{\mathbb{P}}_{i}=0$ $(i=0, \ldots, d)$ meet $V$, where $S_{F}=\frac{\partial F}{\partial u_{00}^{(h)}}$.

In the above theorem $g$ is called the leading differential degree of $V$. From the third statement of the theorem, we see that $V$ intersects with $\mathbb{P}_{\sigma}=0(\sigma=1, \ldots, d)$ and ${ }^{a} \mathbb{P}_{0}^{(l)}=0(l=0, \ldots, h-1)$ in exactly $g$ points. So the leading differential degree satisfies similar properties with the degree for an algebraic variety.

Furthermore, we prove that the four conditions given in Theorem 1.2 are also the sufficient conditions for a differential polynomial $F\left(\mathbf{u}_{0}, \mathbf{u}_{1}, \ldots, \mathbf{u}_{d}\right)$ to be the Chow form for an order un-mixed differential variety or a differential algebraic cycle. As a consequence of this result, we define the Chow quasi-variety for a class of differential algebraic cycles in the sense that each point in the Chow quasi-variety represents a differential algebraic cycle $V$ in that class via the Chow form of $V$. These are clearly generalizations of the algebraic Chow variety and algebraic cycles [13, 16].

Note that both the differential Chow form and the generators of the differential Chow quasi-variety are proven to be differentially homogenous. Further developments of these concepts may need the knowledge of differential projective space [24], which has been ignored to a certain degree in the past.

In [29], Philippon considered the intersection of a variety of dimension $d$ with $d+1$ homogeneous polynomials with generic coefficients and developed the theory for an elimination form which can be regarded as a type of generalized Chow form. In [2, Bost, Gillet, and Soulé further generalized the concept to generalized Chow divisors of cycles and estimated their heights. In this paper, we will introduce the generalized differential Chow form which is roughly defined to be the elimination differential polynomial obtained by intersecting an irreducible differential variety $V$ of dimension $d$ with $d+1$ generic differential hypersurfaces. We show that the generalized differential Chow form satisfies similar properties to that given in Theorem 1.2 . 
As an application of the generalized differential Chow form, we can define the differential resultant. The differential resultant for two nonlinear differential polynomials in one variable was studied by Ritt in [31, p.47]. General differential resultants were defined by Carra' Ferro [5, 6] using Macaulay's definition of an algebraic resultant of polynomials. But, the treatment in [5] is not complete. For instance, the differential resultant for two generic differential polynomials with degree two and order one in one variable is always zero if using the definition in [5]. Differential resultants for linear ordinary differential polynomials were studied by Rueda and Sendra in [36]. In this paper, a rigorous definition for the differential resultant of $n+1$ generic differential polynomials in $n$ variables is given as the generalized differential Chow form of the prime differential ideal $\mathcal{I}=[0]$. In this way, we obtain the following properties for differential resultants, which are similar to that of the Macaulay resultant for $n+1$ algebraic polynomials in $n$ variables.

Theorem 1.3. Let $\mathbb{P}_{i}(i=0, \ldots, n)$ be generic differential polynomials in $n$ differential variables $y_{1}, \ldots, y_{n}$ with orders $s_{i}$, degrees $m_{i}$, and degree zero terms $u_{i 0}$, respectively. Let $R\left(\mathbf{u}_{0}, \mathbf{u}_{1}, \ldots, \mathbf{u}_{n}\right)$ be the differential resultant of $\mathbb{P}_{0}, \ldots, \mathbb{P}_{n}$, where $\mathbf{u}_{i}$ is the set of coefficients of $\mathbb{P}_{i}$. Then

a) $R\left(\mathbf{u}_{0}, \mathbf{u}_{1}, \ldots, \mathbf{u}_{n}\right)$ is differentially homogeneous in each $\mathbf{u}_{i}$ and is of order $h_{i}=s-s_{i}$ in $\mathbf{u}_{i}(i=0, \ldots, n)$ with $s=\sum_{l=0}^{n} s_{l}$.

b) There exist $\xi_{\tau \rho}(\rho=1, \ldots, n)$ in the differential extension fields $\mathcal{F}_{\tau}(\tau=$ $\left.1, \ldots, t_{0}\right)$ of $\mathcal{F}$ such that

$$
R\left(\mathbf{u}_{0}, \mathbf{u}_{1}, \ldots, \mathbf{u}_{n}\right)=A\left(\mathbf{u}_{0}, \mathbf{u}_{1}, \ldots, \mathbf{u}_{n}\right) \prod_{\tau=1}^{t_{0}} \mathbb{P}_{0}\left(\xi_{\tau 1}, \ldots, \xi_{\tau n}\right)^{\left(h_{0}\right)},
$$

where $A\left(\mathbf{u}_{0}, \mathbf{u}_{1}, \ldots, \mathbf{u}_{n}\right)$ is a differential polynomial in $\mathbf{u}_{i}, t_{0}=\operatorname{deg}\left(R, u_{00}^{\left(h_{0}\right)}\right), \mathbb{P}_{0}\left(\xi_{\tau 1}\right.$, $\left.\ldots, \xi_{\tau n}\right)^{\left(h_{0}\right)}$ is the $h_{0}$-th derivative of $\mathbb{P}_{0}\left(\xi_{\tau 1}, \ldots, \xi_{\tau n}\right)$, and $\left(\xi_{\tau 1}, \ldots, \xi_{\tau n}\right)(\tau=$ $\left.1, \ldots, t_{0}\right)$ are certain generic points of the zero-dimensional prime differential ideal $\left[\mathbb{P}_{1}, \ldots, \mathbb{P}_{n}\right]$.

c) The differential resultant can be written as a linear combination of $\mathbb{P}_{i}$ and their derivatives up to the order $s-s_{i}(i=0, \ldots, n)$. Precisely, we have

$$
R\left(\mathbf{u}_{0}, \mathbf{u}_{1}, \ldots, \mathbf{u}_{n}\right)=\sum_{i=0}^{n} \sum_{j=0}^{s-s_{i}} h_{i j} \mathbb{P}_{i}^{(j)} .
$$

In the above expression, $h_{i j} \in \mathcal{F}\langle\mathbf{u}\rangle\left[y_{1}, \ldots, y_{n}, \ldots, y_{1}^{(s)}, \ldots, y_{n}^{(s)}\right]$ have degrees at most $(s n+n)^{2} D^{s n+n}+D(s n+n)$, where $\mathbf{u}=\bigcup_{i=0}^{n} \mathbf{u}_{i} \backslash\left\{u_{00}, \ldots, u_{n 0}\right\}, y_{i}^{(j)}$ is the $j$-th derivative of $y_{i}$, and $D=\max \left\{m_{0}, m_{1}, \ldots, m_{n}\right\}$.

d) Suppose that $\mathbf{u}_{i}(i=0, \ldots, n)$ are differentially specialized to sets $\mathbf{v}_{i}$ of specific elements in $\mathcal{F}$ and $\overline{\mathbb{P}}_{i}(i=0, \ldots, n)$ are obtained by substituting $\mathbf{u}_{i}$ by $\mathbf{v}_{i}$ in $\mathbb{P}_{i}$. If $\overline{\mathbb{P}}_{i}=0(i=0, \ldots, n)$ have a common solution, then $R\left(\mathbf{v}_{0}, \ldots, \mathbf{v}_{n}\right)=0$. On the other hand, if $R\left(\mathbf{v}_{0}, \ldots, \mathbf{v}_{n}\right)=0$ and $S_{R}\left(\mathbf{v}_{0}, \ldots, \mathbf{v}_{n}\right) \neq 0$, then $\overline{\mathbb{P}}_{i}=0(i=0, \ldots, n)$ have a common solution, where $S_{R}=\frac{\partial R}{\partial u_{00}^{\left(h_{0}\right)}}$.

As a prerequisite result, we prove a useful property of differential specializations, which roughly asserts that if a set of differential polynomial functions in a set of indeterminates are differentially dependent, then they are still differentially dependent when the indeterminates are specialized to any concrete values. This property 
plays a key role throughout this paper. The algebraic version of this result is also a key result in algebraic elimination theory ([15, p.168], [43, p.176]).

It is not straightforward to extend the intersection theory for generic polynomials and the theory of Chow forms from the algebraic case to the differential case. Due to the complicated structure of differential polynomials, most proofs in the algebraic case cannot be directly used in the differential case. In particular, we need to consider the orders of differential polynomials, which is not an issue in the algebraic case. For instance, the second property of the differential Chow form in Theorem 1.2 has a different form as its algebraic counterpart.

One of the main tools used in the paper is the theory of characteristic sets developed by Ritt [32, p.47]. The algorithmic character of Ritt's work on differential algebra is mainly due to the usage of characteristic sets. Properties of characteristic sets proved more recently in [1, 7, 10, 12, 43] will also be used in this paper.

The rest of this paper is organized as follows. In Section 2, we will present the notation and preliminary results used in this paper. In Section 3, the intersection theory for generic differential polynomials is given and Theorem 1.1 is proved. In Section 4, the Chow form for an irreducible differential variety is defined and its properties will be proved. Basically, we will prove Theorem 1.2. In Section 5, necessary and sufficient conditions for a differential polynomial to be the Chow form of a differential algebraic cycle is given and the Chow quasi-variety for a class of differential algebraic cycles is defined. In Section 6, we present the theory of the generalized differential Chow form and the differential resultant. Theorem 1.3 will be proved. In Section 7, we present the conclusion and propose several problems for further study.

\section{Preliminaries}

In this section, some basic notation and preliminary results in differential algebra will be given. For more details about differential algebra, please refer to [32, 19, 25, 4, 39.

2.1. Differential polynomial algebra and Kolchin topology. Let $\mathcal{F}$ be a fixed ordinary differential field of characteristic zero, with a derivation $\delta$. An element $c \in \mathcal{F}$ such that $\delta(c)=0$ is called a constant of $\mathcal{F}$. In this paper, unless otherwise indicated, $\delta$ is kept fixed during any discussion and we use primes and exponents $(i)$ to indicate derivatives under $\delta$. Let $\Theta$ denote the free commutative semigroup with unit (written multiplicatively) generated by $\delta$. Throughout the paper, we shall often use the prefix " $\delta$-" as a synonym of "differential" or "differentially".

A typical example of a differential field is $\mathbb{Q}(t)$, which is the field of rational functions in variable $t$ with $\delta=\frac{d}{d t}$.

Let $\mathcal{G}$ be a $\delta$-extension field of $\mathcal{F}$ and $S$ a subset of $\mathcal{G}$. We will denote respectively by $\mathcal{F}[S], \mathcal{F}(S), \mathcal{F}\{S\}$, and $\mathcal{F}\langle S\rangle$ the smallest subring, the smallest subfield, the smallest $\delta$-subring, and the smallest $\delta$-subfield of $\mathcal{G}$ containing $\mathcal{F}$ and $S$. If we denote $\Theta(S)$ to be the smallest subset of $\mathcal{G}$ containing $S$ and stable under $\delta$, we have $\mathcal{F}\{S\}=\mathcal{F}[\Theta(S)]$ and $\mathcal{F}\langle S\rangle=\mathcal{F}(\Theta(S))$. A $\delta$-extension field $\mathcal{G}$ of $\mathcal{F}$ is said to be finitely generated if $\mathcal{G}$ has a finite subset $S$ such that $\mathcal{G}=\mathcal{F}\langle S\rangle$.

A subset $\Sigma$ of a $\delta$-extension field $\mathcal{G}$ of $\mathcal{F}$ is said to be $\delta$-dependent over $\mathcal{F}$ if the set $(\theta \alpha)_{\theta \in \Theta, \alpha \in \Sigma}$ is algebraically dependent over $\mathcal{F}$, and is said to be $\delta$-independent over $\mathcal{F}$, or to be a family of $\delta$-indeterminates over $\mathcal{F}$ (abbr. $\delta$ - $\mathcal{F}$-indeterminates) in 
the contrary case. In the case where $\Sigma$ consists of one element $\alpha$, we say that $\alpha$ is $\delta$ algebraic or $\delta$-transcendental over $\mathcal{F}$, respectively. A maximal subset $\Omega$ of $\mathcal{G}$ which is $\delta$-independent over $\mathcal{F}$ is said to be a $\delta$-transcendence basis of $\mathcal{G}$ over $\mathcal{F}$. We use d.tr.deg $\mathcal{G} / \mathcal{F}$ (see [19, pp.105-109]) to denote the $\delta$-transcendence degree of $\mathcal{G}$ over $\mathcal{F}$, which is the cardinal number of $\Omega$. Considering $\mathcal{F}$ and $\mathcal{G}$ as ordinary algebraic fields, we denote the algebraic transcendence degree of $\mathcal{G}$ over $\mathcal{F}$ by tr.deg $\mathcal{G} / \mathcal{F}$. If $S$ is a set of $\delta$-indeterminates over $\mathcal{F}$, then we call $\mathcal{F}\langle S\rangle$ a pure $\delta$-extension of $\mathcal{F}$.

A homomorphism $\varphi$ from a $\delta$-ring $(\mathcal{R}, \delta)$ to a $\delta_{1}$-ring $\left(\mathcal{S}, \delta_{1}\right)$ is a differential homomorphism if $\varphi \circ \delta=\delta_{1} \circ \varphi$. If $\mathcal{R}_{0}$ is a common $\delta$-subring of $\mathcal{R}$ and $\mathcal{S}$ and if the homomorphism $\varphi$ leaves every element of $\mathcal{R}_{0}$ invariant, it is said to be over $\mathcal{R}_{0}$ and is denoted by $\delta$ - $\mathcal{R}_{0}$-homomorphism. If, in addition, $\mathcal{R}$ is an integral domain and $\mathcal{S}$ is a $\delta$-field, $\varphi$ is called a $\delta$-specialization of $\mathcal{R}$ into $\mathcal{S}$.

A $\delta$-extension field $\mathcal{E}$ of $\mathcal{F}$ is called a universal $\delta$-extension field if for any finitely generated $\delta$-extension field $\mathcal{F}_{1}$ of $\mathcal{F}$ in $\mathcal{E}$ and any finitely generated $\delta$-extension field $\mathcal{F}_{2}$ of $\mathcal{F}_{1}$ not necessarily in $\mathcal{E}, \mathcal{F}_{2}$ can be embedded in $\mathcal{E}$ over $\mathcal{F}_{1}$, i.e. there exists a $\delta$-extension field of $\mathcal{F}_{1}$ in $\mathcal{E}$ that is $\delta$-isomorphic to $\mathcal{F}_{2}$ over $\mathcal{F}_{1}$. Such a $\delta$-universal extension field of $\mathcal{F}$ always exists ([19, Theorem 2, p.134]). By definition, any finitely generated $\delta$-extension field of $\mathcal{F}$ can be embedded over $\mathcal{F}$ into $\mathcal{E}$, and $\mathcal{E}$ is a universal $\delta$-extension field of every finitely generated $\delta$-extension field of $\mathcal{F}$. In particular, for any natural number $n$, we can find in $\mathcal{E}$ a subset of $\mathcal{E}$ of cardinality $n$ whose elements are $\delta$-independent over $\mathcal{F}$. Throughout the present paper, $\mathcal{E}$ stands for a fixed universal $\delta$-extension field of $\mathcal{F}$.

Now suppose $\mathbb{Y}=\left\{y_{1}, y_{2}, \ldots, y_{n}\right\}$ is a set of $\delta$-indeterminates over $\mathcal{E}$. For any $y \in \mathbb{Y}$, denote $\delta^{k} y$ by $y^{(k)}$. The elements of $\mathcal{F}\{\mathbb{Y}\}=\mathcal{F}\left[y_{j}^{(k)}: j=1, \ldots, n ; k \in \mathbb{N}\right]$ are called $\delta$-polynomials over $\mathcal{F}$ in $\mathbb{Y}$, and $\mathcal{F}\{\mathbb{Y}\}$ itself is called the $\delta$-polynomial ring over $\mathcal{F}$ in $\mathbb{Y}$. A $\delta$-polynomial ideal $\mathcal{I}$ in $\mathcal{F}\{\mathbb{Y}\}$ is an ordinary algebraic ideal which is closed under derivation, i.e. $\delta(\mathcal{I}) \subset \mathcal{I}$. In this paper, by $\delta$-ideals we mean $\delta$-polynomial ideals, and by a $\delta$ - $\mathcal{F}$-ideal we mean an ideal in $\mathcal{F}\{\mathbb{Y}\}$. Also, a prime (resp. radical) $\delta$-ideal is a $\delta$-ideal which is prime (resp. radical) as an ordinary algebraic polynomial ideal. For convenience, a prime $\delta$-ideal is assumed not to be the unit ideal in this paper.

By a $\delta$-affine space we mean any one of the sets $\mathcal{E}^{n}(n \in \mathbb{N})$. An element $\eta=$ $\left(\eta_{1}, \ldots, \eta_{n}\right)$ of $\mathcal{E}^{n}$ will be called a point. Let $\Sigma$ be a subset of $\delta$-polynomials in $\mathcal{F}\{\mathbb{Y}\}$. A point $\eta=\left(\eta_{1}, \ldots, \eta_{n}\right) \in \mathcal{E}^{n}$ is called a $\delta$-zero of $\Sigma$ if $f(\eta)=0$ for any $f \in \Sigma$. The set of $\delta$-zeros of $\Sigma$ is denoted by $\mathbb{V}(\Sigma)$, which is called a $\delta$-variety defined over $\mathcal{F}$ (abbr. $\delta$ - $\mathcal{F}$-variety). Also, for any $D \in \mathcal{F}\{\mathbb{Y}\}, \mathbb{V}(\Sigma / D)=\mathbb{V}(\Sigma) \backslash \mathbb{V}(D)$ is called a $\delta$ quasi-variety. For convenience, we also call $\bigcup_{i=1}^{m} \mathbb{V}\left(\Sigma_{i} / D_{i}\right)$ a $\delta$-quasi-variety, where $\Sigma_{i}$ and $D_{i}$ are $\delta$-polynomial sets and $\delta$-polynomials, respectively. The $\delta$-varieties in $\mathcal{E}^{n}$ (resp. the $\delta$ - $\mathcal{F}$-varieties in $\mathcal{E}^{n}$ ) are the closed sets in a topology called the Kolchin topology (resp. the Kolchin $\mathcal{F}$-topology).

For a $\delta$ - $\mathcal{F}$-variety $V$, we denote $\mathbb{I}(V)$ to be the set of all $\delta$-polynomials in $\mathcal{F}\{\mathbb{Y}\}$ that vanish at every point of $V$. Clearly, $\mathbb{I}(V)$ is a radical $\delta$-ideal in $\mathcal{F}\{\mathbb{Y}\}$, and there exists a bijective correspondence between Kolchin $\mathcal{F}$-closed sets and radical $\delta$-ideals in $\mathcal{F}\{\mathbb{Y}\}$. That is, for any $\delta$ - $\mathcal{F}$ variety $V, \mathbb{V}(\mathbb{I}(V))=V$, and for any radical $\delta$-ideal $\mathcal{I}$ in $\mathcal{F}\{\mathbb{Y}\}, \mathbb{I}(\mathbb{V}(\mathcal{I}))=\mathcal{I}$.

Similarly as in algebraic geometry, an $\mathcal{F}$-irreducible $\delta$-variety can be defined, and there is a bijective correspondence between $\mathcal{F}$-irreducible $\delta$-varieties and prime $\delta$-ideals in $\mathcal{F}\{\mathbb{Y}\}$. A point $\eta \in \mathbb{V}(\mathcal{I})$ is called a generic point of a prime $\delta$-ideal 
$\mathcal{I} \subset \mathcal{F}\{\mathbb{Y}\}$ if for any $\delta$-polynomial $p \in \mathcal{F}\{\mathbb{Y}\}$ we have $p(\eta)=0 \Leftrightarrow p \in \mathcal{I}$. It is well known that [32, p.27]

Lemma 2.1. A non-unit $\delta$-ideal is prime if and only if it has a generic point.

By the definition of universal $\delta$-fields, a prime $\delta$-ideal over any finitely generated $\delta$-extension field of $\mathcal{F}$ has a generic point in $\mathcal{E}^{n}$. A point $\eta \in V$ is called a generic point of an irreducible $\delta$-variety $V$ if $\eta$ is a generic point for $\mathbb{I}(V)$.

Notice that irreducibility depends on the base field over which the $\delta$-polynomials are defined. For instance, $\left(\delta y_{1}\right)^{2}-t$ is an irreducible $\delta$-polynomial in $\mathbb{Q}(t)\left\{y_{1}\right\}$ where $\delta=\frac{\partial}{\partial t}$, but it can be factored in $\mathbb{Q}(\sqrt{t})\left\{y_{1}\right\}$. Thus, to emphasize the base field, in the rest of the paper we will use $\mathcal{I}=[\Sigma] \subset \mathcal{G}\{\mathbb{Y}\}$ to denote the $\delta$-ideal generated by $\Sigma$ in $\mathcal{G}\{\mathbb{Y}\}$ and use $\mathcal{G}_{1} \cdot \mathcal{I}$ to denote the $\delta$-ideal generated by $\mathcal{I}$ in $\mathcal{G}_{1}\{\mathbb{Y}\}$ where $\mathcal{G}_{1} \subset \mathcal{E}$ is a $\delta$-extension field of $\mathcal{G}$.

2.2. Characteristic sets of a differential polynomial set. Let $f$ be a $\delta$ polynomial in $\mathcal{F}\{\mathbb{Y}\}$. We define the order of $f$ w.r.t. $y_{i}$ to be the greatest number $k$ such that $y_{i}^{(k)}$ appears effectively in $f$, which is denoted by $\operatorname{ord}\left(f, y_{i}\right)$. If $y_{i}$ does not appear in $f$, then we set $\operatorname{ord}\left(f, y_{i}\right)=-\infty$. The order of $f$ is defined to be $\max _{i} \operatorname{ord}\left(f, y_{i}\right)$, that is, $\operatorname{ord}(f)=\max _{i} \operatorname{ord}\left(f, y_{i}\right)$.

A ranking $\mathscr{R}$ is a total order over $\Theta(\mathbb{Y})$, which is compatible with the derivations over the alphabet:

1) $\delta \theta y_{j}>\theta y_{j}$ for all derivatives $\theta y_{j} \in \Theta(\mathbb{Y})$.

2) $\theta_{1} y_{i}>\theta_{2} y_{j} \Longrightarrow \delta \theta_{1} y_{i}>\delta \theta_{2} y_{j}$ for $\theta_{1} y_{i}, \theta_{2} y_{j} \in \Theta(\mathbb{Y})$.

By convention, $1<\theta y_{j}$ for all $\theta y_{j} \in \Theta(\mathbb{Y})$.

Two important kinds of rankings are the following:

1) Elimination ranking: $y_{i}>y_{j} \Longrightarrow \delta^{k} y_{i}>\delta^{l} y_{j}$ for any $k, l \geq 0$.

2) Orderly ranking: $k>l \Longrightarrow \delta^{k} y_{i}>\delta^{l} y_{j}$, for any $i, j \in\{1,2, \ldots, n\}$.

Let $p$ be a $\delta$-polynomial in $\mathcal{F}\{\mathbb{Y}\}$ and $\mathscr{R}$ a ranking endowed on it. The greatest derivative w.r.t. $\mathscr{R}$ which appears effectively in $p$ is called the leader of $p$, which will be denoted by $u_{p}$ or $\operatorname{ld}(p)$. The two conditions mentioned above imply that the leader of $\theta p$ is $\theta u_{p}$ for $\theta \in \Theta$. Let the degree of $p$ in $u_{p}$ be $d$. We rewrite $p$ as an algebraic polynomial in $u_{p}$. Then

$$
p=I_{d} u_{p}^{d}+I_{d-1} u_{p}^{d-1}+\cdots+I_{0} .
$$

We call $I_{d}$ the initial of $p$ and denote it by $\mathrm{I}_{p}$. The partial derivative of $p$ w.r.t. $u_{p}$ is called the separant of $p$, which will be denoted by $\mathrm{S}_{p}$. Clearly, $\mathrm{S}_{p}$ is the initial of any proper derivative of $p$. The rank of $p$ is $u_{p}^{d}$, and we denote it by $\operatorname{rk}(p)$. For any two $\delta$-polynomials $p, q$ in $\mathcal{F}\{\mathbb{Y}\} \backslash \mathcal{F}, p$ is said to be of lower rank than $q$ if either $u_{p}<u_{q}$ or $u_{p}=u_{q}=u$ and $\operatorname{deg}(p, u)<\operatorname{deg}(q, u)$. By convention, any element of $\mathcal{F}$ is of lower rank than elements of $\mathcal{F}\{\mathbb{Y}\} \backslash \mathcal{F}$. We denote $p \preceq q$ if and only if either $p$ is of lower rank than $q$ or they have the same rank. Clearly, $\preceq$ is a total ordering of $\mathcal{F}\{\mathbb{Y}\}$.

Let $p$ and $q$ be two $\delta$-polynomials and $u_{p}^{d}$ the rank of $p . q$ is said to be partially reduced w.r.t. $p$ if no proper derivatives of $u_{p}$ appear in $q . q$ is said to be reduced w.r.t. $p$ if $q$ is partially reduced w.r.t. $p$ and $\operatorname{deg}\left(q, u_{p}\right)<d$. Let $\mathcal{A}$ be a set of $\delta$-polynomials. $\mathcal{A}$ is said to be an auto-reduced set if each $\delta$-polynomial of $\mathcal{A}$ is reduced w.r.t. any other element of $\mathcal{A}$. Every auto-reduced set is finite.

Let $\mathcal{A}$ be an auto-reduced set. We denote $\mathrm{H}_{\mathcal{A}}$ to be the set of all the initials and separants of $\mathcal{A}$ and $\mathrm{H}_{\mathcal{A}}^{\infty}$ to be the minimal multiplicative set containing $\mathrm{H}_{\mathcal{A}}$. The 
saturation ideal of $\mathcal{A}$ is defined to be

$$
\operatorname{sat}(\mathcal{A})=[\mathcal{A}]: H_{\mathcal{A}}^{\infty}=\left\{p \in \mathcal{F}\{\mathbb{Y}\} \mid \exists h \in H_{\mathcal{A}}^{\infty} \text {, s. t. } h p \in[A]\right\} .
$$

Let $\mathcal{A}=A_{1}, A_{2}, \ldots, A_{s}$ and $\mathcal{B}=B_{1}, B_{2}, \ldots, B_{l}$ be two auto-reduced sets with the $A_{i}, B_{j}$ arranged in nondecreasing ordering. $\mathcal{A}$ is said to be of lower rank than $\mathcal{B}$ if either 1) there is some $k(\leq \min \{s, l\})$ such that for each $i<k, A_{i}$ has the same rank as $B_{i}$, and $A_{k} \prec B_{k}$ or 2) $s>l$, and for each $i \in\{1,2, \ldots, l\}, A_{i}$ has the same rank as $B_{i}$. It is easy to see that the above definition really introduces a partial ordering among all auto-reduced sets. Any sequence of auto-reduced sets steadily decreasing in ordering $\mathcal{A}_{1} \succ \mathcal{A}_{2} \succ \cdots \mathcal{A}_{k} \succ \cdots$ is necessarily finite.

Let $\mathcal{A}=A_{1}, A_{2}, \ldots, A_{t}$ be an auto-reduced set with $\mathrm{S}_{i}$ and $\mathrm{I}_{i}$ as the separant and initial of $A_{i}$, and $f$ any $\delta$-polynomial. Then there exists an algorithm, called Ritt's algorithm of reduction, which reduces $f$ w.r.t. $\mathcal{A}$ to a $\delta$-polynomial $r$ that is reduced w.r.t. $\mathcal{A}$, satisfying the relation

$$
\prod_{i=1}^{t} \mathrm{~S}_{i}^{d_{i}} \mathrm{I}_{i}^{e_{i}} \cdot f \equiv r, \bmod [\mathcal{A}],
$$

for nonnegative integers $d_{i}, e_{i}(i=1,2, \ldots, t)$. We call $r$ the pseudo remainder of $f$ w.r.t. $\mathcal{A}$.

An auto-reduced set $\mathcal{C}$ contained in a $\delta$-polynomial set $\mathcal{S}$ is said to be a characteristic set of $\mathcal{S}$ if $\mathcal{S}$ does not contain any nonzero element reduced w.r.t. $\mathcal{C}$. All the characteristic sets of $\mathcal{S}$ have the same and minimal rank among all auto-reduced sets contained in $\mathcal{S}$. A characteristic set $\mathcal{C}$ of a $\delta$-ideal $\mathcal{J}$ reduces to zero all elements of $\mathcal{J}$. If the $\delta$-ideal is prime, $\mathcal{C}$ reduces to zero only the elements of $\mathcal{J}$ and we have $\mathcal{J}=\operatorname{sat}(\mathcal{C})([19$, Lemma 2, p.167]).

In polynomial algebra, let $\mathcal{A}=A_{1}, A_{2}, \ldots, A_{t}$ be an algebraic auto-reduced set arranged in nondecreasing order. $\mathcal{A}$ is called an irreducible auto-reduced set if for any $1 \leq i \leq t$, there cannot exist any relation of the form

$$
T_{i} A_{i}=B_{i} C_{i}, \bmod \left(A_{1}, \ldots, A_{i-1}\right)
$$

where $B_{i}, C_{i}$ are polynomials with the same leader as $A_{i}, T_{i}$ is a polynomial with a lower leader than $A_{i}$, and $B_{i}, C_{i}, T_{i}$ are reduced w.r.t. $A_{1}, \ldots, A_{i-1}$ ([42]). Equivalently, an algebraic auto-reduced set $\mathcal{A}$ is irreducible if and only if there exist no polynomials $P$ and $Q$ which are reduced w.r.t. $\mathcal{A}$ and $P Q \in \operatorname{asat}(\mathcal{A})=(\mathcal{A}): I_{\mathcal{A}}^{\infty}$, where $I_{\mathcal{A}}^{\infty}$ stands for the set of all products of powers of $\mathrm{I}_{A_{i}}$.

In ordinary differential algebra, we can define an auto-reduced set to be $\mathrm{ir}$ reducible if, when considered as an algebraic auto-reduced set in the underlying polynomial ring, it is irreducible. We have ([32, p.107]).

Theorem 2.2. Let $\mathcal{A}$ be an auto-reduced set. Then a necessary and sufficient condition for $\mathcal{A}$ to be a characteristic set of a prime $\delta$-ideal is that $\mathcal{A}$ is irreducible. Moreover, in the case $\mathcal{A}$ is irreducible, $\operatorname{sat}(\mathcal{A})=[\mathcal{A}]: \mathrm{H}_{\mathcal{A}}^{\infty}$ is prime with $\mathcal{A}$ being a characteristic set of it.

Remark 2.3. A set of $\delta$-polynomials $\mathcal{A}=\left\{A_{1} \ldots, A_{p}\right\}$ is called a $\delta$-chain if the following conditions are satisfied:

1) the leaders of $A_{i}$ are $\delta$-auto-reduced,

2) each $A_{i}$ is partially reduced w.r.t. all the others,

3) no initial of an element of $\mathcal{A}$ is reduced to zero by $\mathcal{A}$. 
Similar properties to auto-reduced sets can be developed for $\delta$-chains [10]. In particular, we can define a $\delta$-characteristic set of a $\delta$-ideal $\mathcal{I}$ to be a $\delta$-chain contained in $\mathcal{I}$ of minimal rank among all the $\delta$-chains contained in $\mathcal{I}$. So, in this paper we will not distinguish auto-reduced sets and $\delta$-chains. Note that we can also use the weak $\delta$-chains introduced in 7 .

2.3. Dimension and order of a prime differential polynomial ideal. Let $\mathcal{I}$ be a prime $\delta$-ideal in $\mathcal{F}\{\mathbb{Y}\}$ and $\xi=\left(\xi_{1}, \ldots, \xi_{n}\right)$ a generic point of $\mathcal{I}$ [19, p.19]. The dimension of $\mathcal{I}$ or $\mathbb{V}(\mathcal{I})$ is defined to be the $\delta$-transcendence degree of $\mathcal{F}\left\langle\xi_{1}, \ldots, \xi_{n}\right\rangle$ over $\mathcal{F}$, that is, $\operatorname{dim}(\mathcal{I})=$ d.tr. $\operatorname{deg} \mathcal{F}\left\langle\xi_{1}, \ldots, \xi_{n}\right\rangle / \mathcal{F}$.

In 32, Ritt gave another definition of the dimension of $\mathcal{I}$. An independent set modulo $\mathcal{I}$ is defined to be a variable set $\mathbb{U} \subset \mathbb{Y}$ such that $\mathcal{I} \cap \mathcal{F}\{\mathbb{U}\}=\{0\}$, and in this case $\mathbb{U}$ is also said to be $\delta$-independent modulo $\mathcal{I}$. A parametric set of $\mathcal{I}$ is a maximal independent set modulo $\mathcal{I}$. Then Ritt defined the dimension of $\mathcal{I}$ to be the cardinal number of its parametric set. Clearly, the two definitions are equivalent.

Definition $2.4([22)$. Let $\mathcal{I}$ be a prime $\delta$-ideal of $\mathcal{F}\{\mathbb{Y}\}$ with a generic point $\eta=\left(\eta_{1}, \ldots, \eta_{n}\right)$. Then there exists a unique numerical polynomial $\omega_{\mathcal{I}}(t)$ such that $\omega_{\mathcal{I}}(t)=\omega_{\eta / \mathcal{F}}(t)=\operatorname{tr} \cdot \operatorname{deg} \mathcal{F}\left(\eta_{i}^{(j)}: i=1, \ldots, n ; j \leq t\right) / \mathcal{F}$ for all sufficiently large $t \in \mathbb{N}$. $\omega_{\mathcal{I}}(t)$ is called the $\delta$-dimension polynomial of $\mathcal{I}$.

We now define the order of a prime $\delta$-ideal $\mathcal{I}$, which is also related with the characteristic set of $\mathcal{I}$.

Definition 2.5. For an auto-reduced set $\mathcal{A}=A_{1}, A_{2}, \ldots, A_{t}$, with $\operatorname{ld}\left(A_{i}\right)=y_{c_{i}}^{\left(o_{i}\right)}$, the order of $\mathcal{A}$ is defined to be $\operatorname{ord}(\mathcal{A})=\sum_{i=1}^{t} o_{i}$, and the set $\mathbb{Y} \backslash\left\{y_{c_{1}}, \ldots, y_{c_{t}}\right\}$ is called a parametric set of $\mathcal{A}$.

Theorem 2.6 ([34, Theorem 13]). Let $\mathcal{I}$ be a prime $\delta$-ideal of dimension $d$. Then the $\delta$-dimension polynomial has the form $\omega_{\mathcal{I}}(t)=d(t+1)+h$, where $h$ is defined to be the order of $\mathcal{I}$ or $\mathbb{V}(\mathcal{I})$, that is, $\operatorname{ord}(\mathcal{I})=h$. Let $\mathcal{A}$ be a characteristic set of $\mathcal{I}$ under any orderly ranking. Then, $\operatorname{ord}(\mathcal{I})=\operatorname{ord}(\mathcal{A})$.

In 32 , Ritt introduced the concept of relative order for a prime $\delta$-ideal w.r.t. a particular parametric set.

Definition 2.7. Let $\mathcal{I}$ be a prime $\delta$-ideal of $\mathcal{F}\{\mathbb{Y}\}, \mathcal{A}$ a characteristic set of $\mathcal{I}$ w.r.t. any elimination ranking, and $\left\{u_{1}, \ldots, u_{d}\right\} \subset \mathbb{Y}$ the parametric set of $\mathcal{A}$. The relative order of $\mathcal{I}$ w.r.t. $\left\{u_{1}, \ldots, u_{d}\right\}$, denoted by $\operatorname{ord}_{u_{1}, \ldots, u_{d}} \mathcal{I}$, is defined to be $\operatorname{ord}(\mathcal{A})$.

The relative order of a prime $\delta$-ideal $\mathcal{I}$ can be computed from its generic points as shown by the following result $([21])$.

Corollary 2.8 ([21]). Let $\mathcal{I}$ be a prime $\delta$-ideal in $\mathcal{F}\{\mathbb{Y}\}$ with a generic point $\left(\xi_{1}, \ldots\right.$, $\left.\xi_{n}\right)$. If $\left\{y_{1}, \ldots, y_{d}\right\}$ is a parametric set of $\mathcal{I}$, then

$$
\operatorname{ord}_{y_{1}, \ldots, y_{d}}(\mathcal{I})=\operatorname{tr} . \operatorname{deg} \mathcal{F}\left\langle\xi_{1}, \ldots, \xi_{d}\right\rangle\left\langle\xi_{d+1}, \ldots, \xi_{n}\right\rangle / \mathcal{F}\left\langle\xi_{1}, \ldots, \xi_{d}\right\rangle .
$$

Ritt's definition of relative order is based on the elimination ranking. Hubert proved that all characteristic sets of $\mathcal{I}$ admitting the same parametric set have the same order 10 .

Theorem 2.9 ([10]). Let $\mathcal{A}$ be a characteristic set of a prime $\delta$-ideal $\mathcal{I}$ in $\mathcal{F}\{\mathbb{Y}\}$ endowed with any ranking. The parametric set $\mathbb{U}$ of $\mathcal{A}$ is a maximal independent 
set modulo $\mathcal{I}$. Its cardinality gives the dimension of $\mathcal{I}$. Furthermore, the order of $\mathcal{I}$ relative to $\mathbb{U}$ is the order of $\mathcal{A}$.

Corollary 2.10. Let $\mathcal{I}$ be a prime $\delta$-ideal with dimension zero and $\mathcal{A}$ a characteristic set of $\mathcal{I}$ w.r.t. any ranking $\mathscr{R}$. Then $\operatorname{ord}(\mathcal{I})=\operatorname{ord}(\mathcal{A})$.

The following result gives the relation between the order and relative order for a prime $\delta$-ideal.

Theorem 2.11. Let $\mathcal{I}$ be a prime $\delta$-ideal in $\mathcal{F}\{\mathbb{Y}\}$. Then $\operatorname{ord}(\mathcal{I})$ is the maximum of all the relative orders of $\mathcal{I}$, that is, $\operatorname{ord}(\mathcal{I})=\max _{\mathbb{U}} \operatorname{ord}_{\mathbb{U}}(\mathcal{I})$, where $\mathbb{U}$ is any parametric set of $\mathcal{I}$.

Proof. Let $\mathcal{C}$ be a characteristic set of $\mathcal{I}$ w.r.t. some orderly ranking. First, we claim that any relative order of $\mathcal{I}$ is less than or equal to $\operatorname{ord}(\mathcal{C})$. Let $\mathbb{U}=\left\{u_{1}, \ldots, u_{q}\right\}$ be any parametric set of $\mathcal{I},\left\{y_{1}, \ldots, y_{p}\right\}(p+q=n)$ the set of the remaining variables, and $\mathcal{B}$ any characteristic set of $\mathcal{I}$ w.r.t. the elimination ranking $u_{1} \prec \cdots \prec u_{q} \prec$ $y_{1} \prec \cdots \prec y_{p}$. By Theorem 2.9] it suffices to prove $\operatorname{ord}_{\mathbb{U}}(\mathcal{I}) \leq \operatorname{ord}(\mathcal{C})$.

Let $\eta=\left(\overline{u_{1}}, \ldots, \overline{u_{q}}, \overline{y_{1}}, \ldots, \overline{y_{p}}\right)$ be a generic point of $\mathcal{I}$. Then for sufficiently large $t$, the $\delta$-dimension polynomial of $\mathcal{I}$ is

$$
\begin{aligned}
& \omega_{\mathcal{I}}(t) \\
= & \omega_{\eta / \mathcal{F}}(t) \\
= & \operatorname{tr} \cdot \operatorname{deg} \mathcal{F}\left(\delta^{s} \overline{u_{i}}, \delta^{k} \overline{y_{j}}: s, k \leq t ; i=1, \ldots, q ; j=1, \ldots, p\right) / \mathcal{F} \\
= & \operatorname{tr} \cdot \operatorname{deg} \mathcal{F}\left(\delta^{s} \overline{u_{i}}: s \leq t\right)\left(\delta^{k} \overline{y_{j}}: k \leq t\right) / \mathcal{F}\left(\delta^{s} \overline{u_{i}}: s \leq t\right)+\operatorname{tr} \cdot \operatorname{deg} \mathcal{F}\left(\delta^{s} \overline{u_{i}}: s \leq t\right) / \mathcal{F} \\
= & q(t+1)+\operatorname{tr} \cdot \operatorname{deg} \mathcal{F}\left(\delta^{s} \overline{u_{i}}: s \leq t\right)\left(\delta^{k} \overline{y_{j}}: k \leq t\right) / \mathcal{F}\left(\delta^{s} \overline{u_{i}}: s \leq t\right) .
\end{aligned}
$$

Since $\omega_{\mathcal{I}}(t)=q(t+1)+\operatorname{ord}(\mathcal{C}), \operatorname{tr} \cdot \operatorname{deg} \mathcal{F}\left(\delta^{s} \overline{u_{i}}: s \leq t\right)\left(\delta^{k} \overline{y_{j}}: k \leq t\right) / \mathcal{F}\left(\delta^{s} \overline{u_{i}}: s \leq\right.$ $t)=\operatorname{ord}(\mathcal{C})$. By Corollary 2.8, we have

$$
\begin{aligned}
& \operatorname{ord}_{\mathbb{U}}(\mathcal{I}) \\
= & \operatorname{tr} \cdot \operatorname{deg} \mathcal{F}\left\langle\overline{u_{1}}, \ldots, \overline{u_{q}}\right\rangle\left(\delta^{k} \overline{y_{l}}: k \geq 0\right) / \mathcal{F}\left\langle\overline{u_{1}}, \ldots, \overline{u_{q}}\right\rangle \\
= & \operatorname{tr} \cdot \operatorname{deg} \mathcal{F}\left\langle\overline{u_{1}}, \ldots, \overline{u_{q}}\right\rangle\left(\delta^{k} \overline{y_{l}}: k \leq t\right) / \mathcal{F}\left\langle\overline{u_{1}}, \ldots, \overline{u_{q}}\right\rangle \quad(\text { for } t \geq \operatorname{ord}(\mathcal{B})) \\
\leq & \operatorname{tr} \cdot \operatorname{deg} \mathcal{F}\left(\delta^{s} \overline{u_{i}}: s \leq t\right)\left(\delta^{k} \overline{y_{j}}: k \leq t\right) / \mathcal{F}\left(\delta^{s} \overline{u_{i}}: s \leq t\right) \\
= & \operatorname{ord}(\mathcal{C}) .
\end{aligned}
$$

Thus, the claim is proved.

Now, let $\mathbb{U}^{*}$ be the parametric set of $\mathcal{C}$. Then, by Theorem 2.9 , $\operatorname{ord}(\mathcal{I})=$ $\operatorname{ord}(C)=\operatorname{ord}_{\mathbb{U}^{*}}(\mathcal{I})$. That is, for any parametric set $\mathbb{U}$ of $\mathcal{I}$, we have $\operatorname{ord}_{\mathbb{U}}(\mathcal{I}) \leq$ $\operatorname{ord}(\mathcal{I})$ and there exists one parametric set $\mathbb{U}^{*}$ of $\mathcal{I}$ such that $\operatorname{ord}_{\mathbb{U}^{*}}(\mathcal{I})=\operatorname{ord}(\mathcal{I})$. As a consequence, $\operatorname{ord}(\mathcal{I})=\max _{\mathbb{U}} \operatorname{ord}_{\mathbb{U}}(\mathcal{I})$.

The following well-known result about the adjoining indeterminates to the base field will be used in this paper [19, 32.

Lemma 2.12. Let $\mathbb{U}=\left\{u_{1}, \ldots, u_{r}\right\} \subset \mathcal{E}$ be a set of $\delta$-F-indeterminates, $\mathcal{I}_{0}$ a prime $\delta$-ideal of dimension d and order $h$ in $\mathcal{F}\{\mathbb{Y}\}$, and $\mathcal{I}=\mathcal{F}\langle\mathbb{U}\rangle \cdot \mathcal{I}_{0}$ the $\delta$-ideal generated by $\mathcal{I}_{0}$ in $\mathcal{F}\langle\mathbb{U}\rangle\{\mathbb{Y}\}$. Then $\mathcal{I}$ is a prime $\delta$-ideal in $\mathcal{F}\langle\mathbb{U}\rangle\{\mathbb{Y}\}$ of dimension $d$ and order $h$ and $\mathcal{I} \cap \mathcal{F}\{\mathbb{Y}\}=\mathcal{I}_{0}$. 
2.4. A property on differential specialization. The following lemma is a key result in algebraic elimination theory, which is used to develop the theory of Chow form ([15, pp.168-169], [43, p.176]). The result is originally given for homogenous polynomials. We will show that it also holds for nonhomogenous polynomials.

Lemma 2.13. Let $P_{i} \in \mathcal{F}[\mathbb{U}, \mathbb{Y}](i=1, \ldots, m)$ be polynomials in the independent indeterminates $\mathbb{U}=\left(u_{1}, \ldots, u_{r}\right)$ and $\mathbb{Y}=\left(y_{1}, \ldots, y_{n}\right)$. Let $\overline{\mathbb{Y}}=\left(\bar{y}_{1}, \ldots, \bar{y}_{n}\right)$, where $\bar{y}_{i}$ are elements of some extension field of $\mathcal{F}$ free from $\mathcal{F}(\mathbb{U}) \sqrt{1}$ If $P_{i}(\mathbb{U}, \overline{\mathbb{Y}})(i=$ $1, \ldots, m)$ are algebraically dependent over $\mathcal{F}(\mathbb{U})$, then for any specialization $\mathbb{U}$ to $\overline{\mathbb{U}}=\left(\bar{u}_{1}, \ldots, \bar{u}_{r}\right) \in \mathcal{F}^{r}, P_{i}(\overline{\mathbb{U}}, \overline{\mathbb{Y}})(i=1, \ldots, m)$ are algebraically dependent over $\mathcal{F}$.

Proof. We sketch the proof for the case $r=1$. Since $P_{i}\left(u_{1}, \overline{\mathbb{Y}}\right)(i=1, \ldots, m)$ are algebraically dependent over $\mathcal{F}\left(u_{1}\right)$, there exists some nonzero polynomial $f \in \mathcal{F}\left(u_{1}\right)\left[z_{1}, \ldots, z_{m}\right]$ which vanishes for $z_{i}=P_{i}\left(u_{1}, \bar{Y}\right)$. By clearing fractions when necessary, we suppose $f \in \mathcal{F}\left[u_{1}, z_{1}, \ldots, z_{m}\right]$. Now specialize $u_{1}$ to $\bar{u}_{1}$ in $f$; then we have $f\left(\bar{u}_{1} ; P_{1}\left(\bar{u}_{1}, \overline{\mathbb{Y}}\right), \ldots, P_{m}\left(\bar{u}_{1}, \overline{\mathbb{Y}}\right)\right)=0$. If $f\left(\bar{u}_{1} ; z_{1}, \ldots, z_{m}\right) \neq 0$, it follows that $P_{i}\left(\bar{u}_{1}, \overline{\mathbb{Y}}\right)(i=1, \ldots, m)$ are algebraically dependent over $\mathcal{F}$. If $f\left(\bar{u}_{1} ; z_{1}, \ldots, z_{m}\right)=$ 0 , then $f\left(u_{1} ; z_{1}, \ldots, z_{m}\right)=\left(u_{1}-\bar{u}_{1}\right)^{l} f_{1}$, where $f_{1}\left(\bar{u}_{1} ; z_{1}, \ldots, z_{m}\right) \neq 0$. Since $f\left(\bar{u}_{1} ; P_{1}\left(\bar{u}_{1}, \overline{\mathbb{Y}}\right), \ldots, P_{m}\left(\bar{u}_{1}, \overline{\mathbb{Y}}\right)\right)=0$, we have $f_{1}\left(\bar{u}_{1} ; P_{1}\left(\bar{u}_{1}, \overline{\mathbb{Y}}\right), \ldots, P_{m}\left(\bar{u}_{1}, \overline{\mathbb{Y}}\right)\right)=0$. Thus, it follows that $P_{i}(\overline{\mathbb{U}}, \bar{Y})(i=1, \ldots, m)$ are algebraically dependent over $\mathcal{F}$.

To generalize the above result to the differential case, we need the following lemma [32, p.35].

Lemma 2.14. Suppose $\mathcal{F}$ contains at least one nonconstant element. If $G \in \mathcal{F}\{u\}$ is a nonzero $\delta$-polynomial with order $r$, then for any nonconstant $\eta \in \mathcal{F}$, there exists an element $c_{0}+c_{1} \eta+c_{2} \eta^{2}+\cdots+c_{r} \eta^{r}$ which does not annul $G$, where $c_{0}, \ldots, c_{r}$ are constants in $\mathcal{F}$.

By arbitrary constants $a_{1}, \ldots, a_{s}$ over $\mathcal{F}$, we mean $a_{1}, \ldots, a_{s}$ are constants in $\mathcal{E}$, which are algebraically independent over $\mathcal{F}$. As a consequence of Lemma 2.14, we have

Corollary 2.15. Suppose $\mathcal{F}$ contains at least one nonconstant element. If $G \in$ $\mathcal{F}\{u\}$ is a nonzero $\delta$-polynomial with order $r$, then for any nonconstant $\eta \in \mathcal{F}$ and arbitrary constants $a_{0}, \ldots, a_{r}$ over $\mathcal{F}, a_{0}+a_{1} \eta+a_{2} \eta^{2}+\cdots+a_{r} \eta^{r}$ does not annul G.

Proof. Suppose the contrary. Let $a_{0}, \ldots, a_{r}$ be arbitrary constants over $\mathcal{F}$ in $\mathcal{E}$ such that $G\left(a_{0}+a_{1} \eta+\cdots+a_{r} \eta^{r}\right)=0$, where $\eta$ is a nonconstant in $\mathcal{F}$. Since $a_{0}, \ldots, a_{r}$ are constants in $\mathcal{E}$ which are algebraically independent over $\mathcal{F}, g\left(a_{0}, \ldots, a_{r}\right)=$ $G\left(a_{0}+a_{1} \eta+\cdots+a_{r} \eta^{r}\right)$ is a polynomial in $\mathcal{F}\left[a_{0}, \ldots, a_{r}\right]$. Now by the hypothesis, $g$ is a zero polynomial. Thus, for any constants $\overline{a_{i}} \in \mathcal{F}(i=0, \ldots, r), g\left(\overline{a_{0}}, \ldots, \overline{a_{r}}\right)=$ $G\left(\overline{a_{0}}+\overline{a_{1}} \eta+\cdots+\overline{a_{r}} \eta^{r}\right)=0$, which contradicts Lemma 2.14 .

Now we prove the following result, which is crucial throughout the paper.

Theorem 2.16. Let $\left\{u_{1}, \ldots, u_{r}\right\} \subset \mathcal{E}$ be a set of $\delta$ - $\mathcal{F}$-indeterminates, and $P_{i}(\mathbb{U}, \mathbb{Y})$ $\in \mathcal{F}\{\mathbb{U}, \mathbb{Y}\}(i=1, \ldots, m) \delta$-polynomials in the $\delta$-F-indeterminates $\mathbb{U}=\left(u_{1}, \ldots, u_{r}\right)$ and $\mathbb{Y}=\left(y_{1}, \ldots, y_{n}\right)$. Let $\overline{\mathbb{Y}}=\left(\bar{y}_{1}, \bar{y}_{2}, \ldots, \bar{y}_{n}\right)$, where $\bar{y}_{i} \in \mathcal{E}$ are $\delta$-free from $\mathcal{F}\langle\mathbb{U}\rangle$.

\footnotetext{
${ }^{1}$ By saying $\overline{\mathbb{Y}}$ is free from $\mathcal{F}(\mathbb{U})$ (resp. $\delta$-free from $\mathcal{F}\langle\mathbb{U}\rangle$ ), we mean that $\mathbb{U}$ is a set of indeterminates over $\mathcal{F}(\overline{\mathbb{Y}})$ (resp. $\delta$-indeterminates over $\mathcal{F}\langle\overline{\mathbb{Y}}\rangle)$.
} 
If $P_{i}(\mathbb{U}, \overline{\mathbb{Y}})(i=1, \ldots, m)$ are $\delta$-dependent over $\mathcal{F}\langle\mathbb{U}\rangle$, then for any specialization $\mathbb{U}$ to $\overline{\mathbb{U}}$ in $\mathcal{F}, P_{i}(\overline{\mathbb{U}}, \overline{\mathbb{Y}})(i=1, \ldots, m)$ are $\delta$-F-dependent.

Proof. It suffices to prove the case $r=1$. Denote $u_{1}$ by $u$. First, we suppose $\mathcal{F}$ contains at least one nonconstant element.

Since $P_{i}(u, \bar{Y})(i=1, \ldots, m)$ are $\delta$-dependent over $\mathcal{F}\langle u\rangle$, there exists a nonzero $G\left(z_{1}, \ldots, z_{m}\right) \in \mathcal{F}\langle u\rangle\left\{z_{1}, \ldots, z_{m}\right\}$ such that $G\left(P_{1}(u, \overline{\mathbb{Y}}), \ldots, P_{m}(u, \bar{Y})\right)=0$. We can take $G \in \mathcal{F}\left\{u, z_{1}, \ldots, z_{m}\right\}$ by clearing denominators when necessary.

Since $G\left(u, z_{1}, \ldots, z_{m}\right) \neq 0$, by Corollary 2.15, for any nonconstant $\eta \in \mathcal{F}$ and arbitrary constants $c_{0}, \ldots, c_{s}(s=\operatorname{ord}(G, u))$ over $\mathcal{F}\langle\overline{\mathbb{Y}}\rangle, G\left(u^{*}, z_{1}, \ldots, z_{m}\right) \neq 0$ where $u^{*}=\sum_{i=0}^{s} c_{i} \eta^{i}$. Since $G\left(u, P_{1}(u, \overline{\mathbb{Y}}), \ldots, P_{m}(u, \overline{\mathbb{Y}})\right)=0$ and $u$ is a $\delta$ indeterminate over $\mathcal{F}\langle\overline{\mathbb{Y}}\rangle$, when $u$ is specialized to $u^{*}$, we have $G\left(u^{*}, P_{1}\left(u^{*}, \overline{\mathbb{Y}}\right), \ldots\right.$, $\left.P_{m}\left(u^{*}, \bar{Y}\right)\right)=0$. Regarding $G$ as an algebraic polynomial over $\mathcal{F}$ in $c_{i}(i=0, \ldots, s)$ and $z_{i}^{(j)}(i=1, \ldots, m ; j \geq 0)$ which appear effectively, we have

$$
G\left(c_{0}, \ldots, c_{s}, \ldots, z_{i}^{(j)}, \ldots\right) \neq 0,
$$

while

$$
G\left(c_{0}, \ldots, c_{s}, \ldots,\left(P_{i}\left(u^{*}, \bar{Y}\right)\right)^{(j)}, \ldots\right)=0 .
$$

So $\left.P_{i}\left(u^{*}, \bar{Y}\right)\right)^{(j)}(i=1, \ldots, m ; j \geq 0)$ are algebraically dependent over $\mathcal{F}\left(c_{0}, \ldots, c_{s}\right)$, by Lemma 2.13, when the $c_{i}$ are specialized to constants $\bar{c}_{i}$ in $\mathcal{F}$, the corresponding $P_{i}\left(\overline{u^{*}}, \overline{\mathbb{Y}}\right)^{(j)}(i=1, \ldots, m)$ are algebraically dependent over $\mathcal{F}$, where $\overline{u^{*}}=\sum_{i=0}^{s} \bar{c}_{i} \eta^{i}$. That is, $P_{i}\left(\overline{u^{*}}, \overline{\mathbb{Y}}\right)(i=1, \ldots, m)$ are $\delta$-dependent over $\mathcal{F}$.

To complete the proof, if $\bar{u}$ is a nonconstant, as above we take $\eta=\bar{u}$, and specialize $c_{1} \rightarrow 1$ and other $c_{i}$ to zero. Otherwise we take $\eta$ as an arbitrary nonconstant and specialize $c_{0} \rightarrow \bar{u}$ and other $c_{i}$ to zero. Then in either case, $u$ is specialized to $\bar{u}$, and we have completed the proof in the case where $\mathcal{F}$ contains at least one nonconstant element.

If $\mathcal{F}$ consists of constant elements, take $v \in \mathcal{E}$ to be a $\delta$-indeterminate over $\mathcal{F}\langle\mathbb{U}, \bar{Y}\rangle$. Now we consider in the $\delta$-field $\mathcal{F}\langle v\rangle$. Following the first case, for any specialization $\mathbb{U}$ to $\overline{\mathbb{U}} \subset \mathcal{F}$, we can show that $P_{i}(\overline{\mathbb{U}}, \overline{\mathbb{Y}})(i=1, \ldots, m)$ are $\delta$-dependent over $\mathcal{F}\langle v\rangle$. Since $v$ is a $\delta$-indeterminate over $\mathcal{F}\langle\overline{\mathbb{Y}}\rangle, P_{i}(\overline{\mathbb{U}}, \overline{\mathbb{Y}})(i=1, \ldots, m)$ are $\delta$-dependent over $\mathcal{F}$.

From the proof above, we can obtain the following result easily:

Corollary 2.17. Let $\left\{u_{1}, \ldots, u_{r}\right\} \subset \mathcal{E}$ be a set of $\delta$-F $\mathcal{F}$-indeterminates, and $P_{i}(\mathbb{U}, \mathbb{Y})$ $\in \mathcal{F}\{\mathbb{U}, \mathbb{Y}\}(i=1, \ldots, m) \delta$-polynomials in the $\delta$-indeterminates $\mathbb{U}=\left(u_{1}, \ldots, u_{r}\right)$ and $\mathbb{Y}=\left(y_{1}, \ldots, y_{n}\right)$. Let $\overline{\mathbb{Y}}=\left(\bar{y}_{1}, \bar{y}_{2}, \ldots, \bar{y}_{n}\right)$, where $\bar{y}_{i} \in \mathcal{E}$ are free from $\mathcal{F}\langle\mathbb{U}\rangle$. If the set $\left(P_{i}(\mathbb{U}, \bar{Y})\right)^{\left(\sigma_{i j}\right)}\left(i=1, \ldots, m ; j=1, \ldots, n_{i}\right)$ are algebraically dependent over $\mathcal{F}\langle\mathbb{U}\rangle$, then for any specialization $\mathbb{U}$ to $\overline{\mathbb{U}}$ in $\mathcal{F},\left(P_{i}(\overline{\mathbb{U}}, \overline{\mathbb{Y}})\right)^{\left(\sigma_{i j}\right)}(i=1, \ldots, m ; j=$ $\left.1, \ldots, n_{i}\right)$ are algebraically dependent over $\mathcal{F}$.

Now we give an example to illustrate the proof of Theorem 2.16 .

Example 2.18. In this example $r=m=n=1$ and $\mathcal{F}=\mathbb{Q}(t)$ with $\delta=\frac{d}{d t}$. Let $P(u, y)=u y$. Suppose that $\xi$ is a generic point of $\mathcal{I}=\operatorname{sat}\left(y^{\prime 2}-2 y\right) \subset \mathbb{Q}(t)\{y\}$ that is $\delta$-free from $\mathbb{Q}(t)\langle u\rangle$. Let $G(u ; z)=u^{\prime 2} z^{\prime \prime 2}+2 u\left(u^{\prime 2}-u u^{\prime \prime}\right) z^{\prime \prime}-2 u^{\prime} u^{\prime \prime} z^{\prime} z^{\prime \prime}+$ $u^{\prime \prime 2} z^{\prime 2}+z^{\prime}\left(-8 u^{\prime 3}+6 u u^{\prime} u^{\prime \prime}\right)-3 u^{2} u^{\prime 2}+2 u^{3} u^{\prime \prime} \in \mathbb{Q}(t)\{u, z\}$. Let $\bar{y}=\xi$. It is easy to verify that $G(u ; z) \neq 0$ and $G(u ; P(u, \bar{y}))=0$. That is, $P(u, \bar{y})$ is $\delta$-algebraic over $\mathbb{Q}(t)\langle u\rangle$. We now proceed to show that when $u$ is specialized to $\bar{u}=1, P(\bar{u}, \bar{y})$ is $\delta$-algebraic over $\mathbb{Q}(t)$. 
Clearly, $G(\bar{u} ; z)=0$. In the algebraic case, following the proof of Lemma 2.13. $u-\bar{u}$ must be a factor of $G(u ; z)$. Removing all factors of the form $u-\bar{u}$, we obtain a new $\delta$-polynomial $G_{1}(u ; z)$ which still satisfies $G_{1}(u ; P(u, \bar{y}))=0$ and $G_{1}(\bar{u} ; z) \neq 0$. But, in the differential case, $G(u ; z)$ may not contain any factor involving $u-\bar{u}$ and its derivatives.

Follow the steps in Theorem 2.16 and let $c_{0}, c_{1}, c_{2}$ be arbitrary constants over $\mathbb{Q}(t)\langle\xi\rangle$ and $\eta=t$. Denote $u^{*}=c_{0}+c_{1} t+c_{2} t^{2}$. Then $G\left(u^{*} ; z\right)=-40 z^{\prime} c_{2}^{3} t^{3}-6 c_{0} c_{1}^{3} t-$ $12 c_{0} c_{2}^{3} t^{4}-14 c_{1}^{3} t^{3} c_{2}-27 c_{1}^{2} t^{4} c_{2}^{2}+4 z^{\prime 2} c_{2}^{2}-8 z^{\prime} c_{1}^{3}-3 c_{0}^{2} c_{1}^{2}-3 c_{1}^{4} t^{2}-8 c_{2}^{4} t^{6}+4 c_{0}^{3} c_{2}+z^{\prime \prime 2} c_{1}^{2}-$ $36 z^{\prime} c_{1}^{2} c_{2} t-60 z^{\prime} c_{1} c_{2}^{2} t^{2}+12 z^{\prime} c_{0} c_{1} c_{2}+24 z^{\prime} c_{0} c_{2}^{2} t-18 c_{0} c_{1}^{2} t^{2} c_{2}-24 c_{0} c_{1} t^{3} c_{2}^{2}+4 z^{\prime \prime 2} c_{1} c_{2} t+$ $6 z^{\prime \prime} c_{1}^{2} t^{2} c_{2}+8 z^{\prime \prime} c_{1} t^{3} c_{2}^{2}-4 z^{\prime} z^{\prime \prime} c_{1} c_{2}-8 z^{\prime} z^{\prime \prime} c_{2}^{2} t-24 c_{1} t^{5} c_{2}^{3}+2 z^{\prime \prime} c_{0} c_{1}^{2}-4 z^{\prime \prime} c_{0}^{2} c_{2}+$ $4 z^{\prime \prime 2} c_{2}^{2} t^{2}+2 z^{\prime \prime} c_{1}^{3} t+4 z^{\prime \prime} c_{2}^{3} t^{4} \neq 0$ and $G\left(u^{*} ; P\left(u^{*}, \bar{y}\right)\right)=0$. Regard $G\left(u^{*} ; z\right)$ as an algebraic polynomial in $\mathbb{Q}(t)\left[c_{0}, c_{1}, c_{2} ; z, z^{\prime}, z^{\prime \prime}\right]$, denoted by $g\left(c_{0}, c_{1}, c_{2} ; z, z^{\prime}, z^{\prime \prime}\right)$. Then $P\left(u^{*}, \bar{y}\right), P\left(u^{*}, \bar{y}\right)^{\prime}, P\left(u^{*}, \bar{y}\right)^{\prime \prime}$ are algebraically dependent over $\mathbb{Q}(t)\left(c_{0}, c_{1}, c_{2}\right)$. So the problem is converted to an algebraic one, and we can use Lemma 2.13 to solve it.

To be more precise, first, specialize $c_{2}$ to 0 ; we obtain $g\left(c_{0}, c_{1}, 0 ; z, z^{\prime}, z^{\prime \prime}\right)=$ $z^{\prime \prime 2} c_{1}^{2}+2 z^{\prime \prime} c_{0} c_{1}^{2}+2 z^{\prime \prime} c_{1}^{3} t-6 c_{0} c_{1}^{3} t-8 z^{\prime} c_{1}^{3}-3 c_{0}^{2} c_{1}^{2}-3 c_{1}^{4} t^{2} \neq 0$ and $g\left(c_{0}, c_{1}, 0 ; P\left(c_{0}+\right.\right.$ $\left.\left.c_{1} t, \bar{y}\right), P\left(c_{0}+c_{1} t, \bar{y}\right)^{\prime}, P\left(c_{0}+c_{1} t, \bar{y}\right)^{\prime \prime}\right)=0$. Then specialize $c_{1}$ to 0 ; we obtain $g\left(c_{0}, 0,0 ; z, z^{\prime}, z^{\prime \prime}\right)=0$ and $g\left(c_{0}, c_{1}, 0 ; z, z^{\prime}, z^{\prime \prime}\right)=c_{1}^{2} g_{1}$, where $g_{1}=z^{\prime \prime 2}+2 z^{\prime \prime} c_{0}+$ $2 z^{\prime \prime} c_{1} t-6 c_{0} c_{1} t-8 z^{\prime} c_{1}-3 c_{0}^{2}-3 c_{1}^{2} t^{2}$. Clearly, $g_{1}\left(c_{0}, c_{1} ; P\left(c_{0}+c_{1} t, \bar{y}\right), P\left(c_{0}+\right.\right.$ $\left.\left.c_{1} t, \bar{y}\right)^{\prime}, P\left(c_{0}+c_{1} t, \bar{y}\right)^{\prime \prime}\right)=0$. Specialize $c_{1}$ to 0 in $g_{1}, \bar{g}_{1}=z^{\prime \prime 2}+2 z^{\prime \prime} c_{0}-3 c_{0}^{2}$, while $\bar{g}_{1}\left(c_{0} ; P\left(c_{0}, \bar{y}\right), P\left(c_{0}, \bar{y}\right)^{\prime}, P\left(c_{0}, \bar{y}\right)^{\prime \prime}\right)=0$. Now specialize $c_{0}$ to $\bar{u}=1$ in $\bar{g}_{1}$; we obtain $g_{2}\left(z, z^{\prime}, z^{\prime \prime}\right)=z^{\prime \prime 2}+2 z^{\prime \prime}-3$. Clearly, $g_{2}\left(\xi, \xi^{\prime}, \xi^{\prime \prime}\right)=0$, where $\xi=P(\bar{u}, \bar{y})$. Thus, $P(\bar{u}, \bar{y})$ is $\delta$-algebraic over $\mathbb{Q}(t)$. Also, $P(\bar{u}, \bar{y}), P(\bar{u}, \bar{y})^{\prime}$ and $P(\bar{u}, \bar{y})^{\prime \prime}$ are algebraically dependent over $\mathbb{Q}(t)$.

\section{INTERSECTION THEORY FOR GENERIC DIFFERENTIAL POLYNOMIALS}

In this section, we will develop an intersection theory for generic $\delta$-polynomials by proving Theorem 1.1. As a consequence, the dimension conjecture is shown to be true for generic $\delta$-polynomials. These results will also be used in Sections 4 and 6 to determine the order of the Chow form.

3.1. Generic dimension theorem. In this section, we will show that the dimension conjecture is valid for certain generic $\delta$-polynomials. To prove the dimension conjecture in the general case, one simple idea is to generalize the following theorem ([19, p.43]) in algebra to the differential case.

Theorem 3.1. Let $\mathcal{I}$ be a prime ideal of dimension $d>0$ and $f \in \mathcal{F}[\mathbb{Y}]$. If $(\mathcal{I}, f) \neq(1)$, then every prime component of $\mathbb{V}(\mathcal{I}, f)$ has dimension not less than $d-1$. Moreover, if $f$ is not in $\mathcal{I}$, then every prime component of $\mathbb{V}(\mathcal{I}, f)$ has dimension $d-1$.

Unfortunately, in the differential case, the above theorem does not hold. Ritt gave the following counterexample.

Example 3.2 ([32, p.133]). $p=y_{1}^{5}-y_{2}^{5}+y_{3}\left(y_{1} y_{2}^{\prime}-y_{2} y_{1}^{\prime}\right)^{2} \in \mathcal{F}\left\{y_{1}, y_{2}, y_{3}\right\}$ and $f=y_{3}$, where $\mathcal{F}$ is the field of complex numbers. Then sat $(p)$ is a prime $\delta$-ideal of dimension two. But $\sqrt{[\operatorname{sat}(p), f]}=\left[y_{1}, y_{2}, y_{3}\right]$, which is a prime $\delta$-ideal of dimension zero. 
It could also happen that when adding a $\delta$-polynomial to a prime $\delta$-ideal to which it does not belong, the dimension is still the same.

Example 3.3. Let $p=y_{1}^{\prime} y_{2}^{\prime \prime}-y_{1}^{\prime \prime} y_{2}^{\prime}$. Then $\operatorname{sat}(p)=[p]: y_{1}^{\prime \infty}$ is a prime $\delta$-ideal of dimension one. It is clear that $y_{2}^{\prime} \notin \operatorname{sat}(p)$ and $\left[\operatorname{sat}(p), y_{2}^{\prime}\right]=\left[y_{2}^{\prime}\right]$ is still a prime $\delta$-ideal of dimension one.

In this section, we will prove that Theorem 3.1 is valid for certain generic $\delta$ polynomials, which will lead to the solution of the dimension conjecture in these generic cases.

Definition 3.4. Let $\mathrm{m}_{s, r}$ be the set of all $\delta$-monomials in $\mathcal{F}\{\mathbb{Y}\}$ of order $\leq s$ and degree $\leq r$. Let $\mathbb{U}=\left\{u_{m}\right\}_{m \in \mathrm{m}_{s, r}}$ be a set of elements of $\mathcal{E}$ that are $\delta$ - $\mathcal{F}$ indeterminates. Then,

$$
f=\sum_{m \in m_{s, r}} u_{m} m
$$

is called a generic $\delta$-polynomial of order $s$ and degree $r$. If $s=0$, then $f$ is also called a generic polynomial of degree $r$. A generic $\delta$-hypersurface is the set of zeros of a generic $\delta$-polynomial.

We use $\mathbf{u}_{f}$ to denote the set of coefficients of a generic $\delta$-polynomial $f$ and

$$
\widetilde{\mathbf{u}}_{f}=\mathbf{u}_{f} \backslash\left\{u_{0}\right\},
$$

where $u_{0}$ is the degree zero term of $f$. By saying that a point $\eta \in \mathcal{E}^{n}$ is free from the pure $\delta$-extension field $\mathcal{F}\left\langle\mathbf{u}_{f}\right\rangle$ over $\mathcal{F}$, we mean $\mathbf{u}_{f}$ are $\delta$ - $\mathcal{F}\langle\eta\rangle$-indeterminates.

Throughout the paper, a generic $\delta$-polynomial is assumed to be of degree greater than zero.

Lemma 3.5. Let $\mathcal{I}$ be a prime $\delta$-ideal in $\mathcal{F}\{\mathbb{Y}\}$ with dimension $d$ and $f$ a generic $\delta$-polynomial. Then $\mathcal{I}_{0}=[\mathcal{I}, f]$ is a prime $\delta$-ideal in $\mathcal{F}\left\langle\widetilde{\mathbf{u}}_{f}\right\rangle\left\{\mathbb{Y}, u_{0}\right\}$ with dimension $d$, where $\widetilde{\mathbf{u}}_{f}$ is defined in (3.1). Furthermore, $\mathcal{I}_{0} \cap \mathcal{F}\left\langle\widetilde{\mathbf{u}}_{f}\right\rangle\left\{u_{0}\right\}=\{0\}$ if and only if $d>0$.

Proof. Let $\xi=\left(\xi_{1}, \ldots, \xi_{n}\right)$ be a generic point of $\mathcal{I}$ over $\mathcal{F}$ that is free from $\mathcal{F}\left\langle\mathbf{u}_{f}\right\rangle$ and $f=u_{0}+f_{0}$, where $f_{0}=\sum_{\operatorname{deg}(m) \geq 1} u_{m} m$. We claim that $\left(\xi_{1}, \ldots, \xi_{n},-f_{0}(\xi)\right)$ is a generic point of $\mathcal{I}_{0}$ over $\mathcal{F}\left\langle\widetilde{\mathbf{u}}_{f}\right\rangle$. Thus, it follows that $\mathcal{I}_{0}$ is a prime $\delta$-ideal by Lemma 2.1 .

Clearly, $\left(\xi_{1}, \ldots, \xi_{n},-f_{0}(\xi)\right)$ is a zero of $\mathcal{I}_{0}$. Let $g$ be any $\delta$-polynomial in $\mathcal{F}\left\langle\widetilde{\mathbf{u}}_{f}\right\rangle\left\{y_{1}, \ldots, y_{n}, u_{0}\right\}$ which vanishes at $\left(\xi_{1}, \ldots, \xi_{n},-f_{0}(\xi)\right)$. Regarding $u_{0}$ as the leader of $f$, suppose the pseudo remainder of $g$ w.r.t. $f$ is $g_{1}$. Then we have

$$
g \equiv g_{1}, \bmod [f],
$$

where $g_{1} \in \mathcal{F}\left\langle\widetilde{\mathbf{u}}_{f}\right\rangle\left\{y_{1}, \ldots, y_{n}\right\}$. From the above expression, $g_{1}(\xi)=0$. Since $\xi$ is also a generic point of $\mathcal{F}\left\langle\widetilde{\mathbf{u}}_{f}\right\rangle \cdot \mathcal{I}$ over $\mathcal{F}\left\langle\widetilde{\mathbf{u}}_{f}\right\rangle, g_{1} \in \mathcal{F}\left\langle\widetilde{\mathbf{u}}_{f}\right\rangle \cdot \mathcal{I}$. Thus, $g \in \mathcal{I}_{0}$, and it follows that $\left(\xi_{1}, \ldots, \xi_{n},-f_{0}(\xi)\right)$ is a generic point of $\mathcal{I}_{0}$ over $\mathcal{F}\left\langle\widetilde{\mathbf{u}}_{f}\right\rangle$ and $\mathcal{I}_{0}$ is prime.

By Lemma 2.12,

$$
\begin{aligned}
\operatorname{dim} \mathcal{I}_{0} & =\text { d.tr.deg } \mathcal{F}\left\langle\widetilde{\mathbf{u}}_{f}\right\rangle\left\langle\xi_{1}, \ldots, \xi_{n},-f_{0}(\xi)\right\rangle / \mathcal{F}\left\langle\widetilde{\mathbf{u}}_{f}\right\rangle \\
& =\text { d.tr.deg } \mathcal{F}\left\langle\widetilde{\mathbf{u}}_{f}\right\rangle\left\langle\xi_{1}, \ldots, \xi_{n}\right\rangle / \mathcal{F}\left\langle\widetilde{\mathbf{u}}_{f}\right\rangle \\
& =\text { d.tr.deg } \mathcal{F}\left\langle\xi_{1}, \ldots, \xi_{n}\right\rangle / \mathcal{F}=d .
\end{aligned}
$$


Now consider the second part of the lemma. If $d=0$, then $\operatorname{dim} \mathcal{I}_{0}=0$, so $\mathcal{I}_{0} \cap \mathcal{F}\left\langle\widetilde{\mathbf{u}}_{f}\right\rangle\left\{u_{0}\right\} \neq\{0\}$. Thus, if $\mathcal{I}_{0} \cap \mathcal{F}\left\langle\widetilde{\mathbf{u}}_{f}\right\rangle\left\{u_{0}\right\}=\{0\}$, then $d>0$. It remains to show that if $d>0$, then $\mathcal{I}_{0} \cap \mathcal{F}\left\langle\widetilde{\mathbf{u}}_{f}\right\rangle\left\{u_{0}\right\}=\{0\}$. Suppose the contrary; then there exists a nonzero $\delta$-polynomial $p\left(\widetilde{\mathbf{u}}_{f}, u_{0}\right) \in \mathcal{I}_{0} \cap \mathcal{F}\left\{\widetilde{\mathbf{u}}_{f}, u_{0}\right\}$. So $p\left(\widetilde{\mathbf{u}}_{f},-f_{0}(\xi)\right)=0$. Then, $\phi=-f_{0}(\xi)$ is $\delta$-algebraic over $\mathcal{F}\left\langle\widetilde{\mathbf{u}}_{f}\right\rangle$. Denote the coefficient of any monomial $y_{i}$ in $f$ to be $u_{i 0}$. So for any fixed $i$ when $u_{i 0}$ is specialized to -1 and all the other $u \in \widetilde{\mathbf{u}}_{f}$ specialized to zero, by Theorem 2.16, we conclude that $\bar{\phi}=\xi_{i}(i=1, \ldots, n)$ is $\delta$-algebraic over $\mathcal{F}$, which contradicts the fact that $\mathcal{I}$ has a positive dimension. So $\mathcal{I}_{0} \cap \mathcal{F}\left\langle\widetilde{\mathbf{u}}_{f}\right\rangle\left\{u_{0}\right\}=\{0\}$.

Now, we will prove the first key result of this paper, which shows that by adding a generic $\delta$-polynomial to a prime $\delta$-ideal, the new $\delta$-ideal is still prime and its dimension decreases by one. This is generally not valid if the $\delta$-polynomial is not generic, as shown in Examples 3.2 and 3.3 .

Theorem 3.6. Let $\mathcal{I}$ be a prime $\delta$-ideal in $\mathcal{F}\{\mathbb{Y}\}$ with dimension $d$ and $f$ a generic $\delta$-polynomial. If $d>0$, then $\mathcal{I}_{1}=[\mathcal{I}, f]$ is a prime $\delta$-ideal in $\mathcal{F}\left\langle\mathbf{u}_{f}\right\rangle\{\mathbb{Y}\}$ with dimension $d-1$. Also, if $d=0$, then $\mathcal{I}_{1}$ is the unit ideal in $\mathcal{F}\left\langle\mathbf{u}_{f}\right\rangle\{\mathbb{Y}\}$.

Proof. First, we consider the case $d>0$. Let $\left(\xi_{1}, \ldots, \xi_{n}\right)$ be a generic point of $\mathcal{I}$ over $\mathcal{F}$ that is free from the pure $\delta$-extension field $\mathcal{F}\left\langle\mathbf{u}_{f}\right\rangle$ over $\mathcal{F}$ and $f=u_{0}+f_{0}$ where $f_{0}=\sum_{\operatorname{deg}(m) \geq 1} u_{m} m$. By Lemma 3.5. $\mathcal{I}_{0}=[\mathcal{I}, f] \subset \mathcal{F}\left\langle\widetilde{\mathbf{u}}_{f}\right\rangle\left\{\mathbb{Y}, u_{0}\right\}$ is a prime $\delta$-ideal, where $\widetilde{\mathbf{u}}_{f}$ is defined in (3.1). By Lemma 3.5, $\mathcal{I}_{0} \cap \mathcal{F}\left\langle\widetilde{\mathbf{u}}_{f}\right\rangle\left\{u_{0}\right\}=\{0\}$. So $\mathcal{I}_{1}=\left[\mathcal{I}_{0}\right]$ in $\mathcal{F}\left\langle\mathbf{u}_{f}\right\rangle\{\mathbb{Y}\}$ is not the unit ideal, for if not, $1 \in \mathcal{I}_{1}$. Then there exist $\delta$-polynomials $g_{i} \in \mathcal{I} \subset \mathcal{F}\{\mathbb{Y}\}, H_{i j}, G_{k} \in \mathcal{F}\left\langle\mathbf{u}_{f}\right\rangle\{\mathbb{Y}\}$ such that $1=\sum_{i, j} H_{i j} g_{i}^{(j)}+\sum_{k} G_{k} f^{(k)}$. By clearing denominators in the above expression, we obtain a nonzero $\delta$-polynomial in $\mathcal{I}_{0} \cap \mathcal{F}\left\{\mathbf{u}_{f}\right\}$, a contradiction.

Now we claim that $\mathcal{I}_{1}$ is prime and $\mathcal{I}_{1} \cap \mathcal{F}\left\langle\widetilde{\mathbf{u}}_{f}\right\rangle\left\{\mathbb{Y}, u_{0}\right\}=\mathcal{I}_{0}$. Suppose $g, h \in$ $\mathcal{F}\left\langle\mathbf{u}_{f}\right\rangle\{\mathbb{Y}\}$ and $g h \in \mathcal{I}_{1}$. By collecting denominators, there exist $D_{1}, D_{2} \in \mathcal{F}\left\{\mathbf{u}_{f}\right\}$ such that $D_{1} g, D_{2} h \in \mathcal{F}\left\{\mathbf{u}_{f}, \mathbb{Y}\right\}$, and $\left(D_{1} g\right) \cdot\left(D_{2} h\right)=D_{1} D_{2}(g h) \in \mathcal{I}_{1}$ still holds. Similar to the procedure above, we can find a nonzero $D \in \mathcal{F}\left\{\mathbf{u}_{f}\right\}$ such that $D \cdot\left(D_{1} g\right) \cdot\left(D_{2} h\right) \in \mathcal{I}_{0}$. Since $\mathcal{I}_{0}$ is prime and $\mathcal{I}_{0} \cap \mathcal{F}\left\langle\widetilde{\mathbf{u}}_{f}\right\rangle\left\{u_{0}\right\}=\{0\}, D_{1} g \in \mathcal{I}_{0}$ or $D_{2} h \in \mathcal{I}_{0}$. It follows that $g \in \mathcal{I}_{1}$ or $h \in \mathcal{I}_{1}$. Since $\mathcal{I}_{1}$ is not the unit ideal, $\mathcal{I}_{1}$ is prime. Also, for any $g \in \mathcal{I}_{1} \cap \mathcal{F}\left\langle\widetilde{\mathbf{u}}_{f}\right\rangle\left\{\mathbb{Y}, u_{0}\right\}$, there exists some $D \in \mathcal{F}\left\{\mathbf{u}_{f}\right\}$ such that $D g \in \mathcal{I}_{0}$, so $g \in \mathcal{I}_{0}$. Thus, $\mathcal{I}_{1} \cap \mathcal{F}\left\langle\widetilde{\mathbf{u}}_{f}\right\rangle\left\{\mathbb{Y}, u_{0}\right\}=\mathcal{I}_{0}$.

Suppose $\xi_{1}, \ldots, \xi_{d}$ are $\delta$-independent over $\mathcal{F}$. Then, $\left\{y_{1}, \ldots, y_{d}\right\}$ is a parametric set of $\mathcal{I}$. Thus each $y_{d+i}(i=1, \ldots, n-d)$ is $\delta$-dependent with $y_{1}, \ldots, y_{d}$ modulo $\mathcal{I}_{1}$, since $\mathcal{I} \subset \mathcal{I}_{1}$. By Lemma 3.5, $\operatorname{dim} \mathcal{I}_{0}=d$. Then $u_{0}, y_{1}, \ldots, y_{d}$ are $\delta$-dependent modulo $\mathcal{I}_{0}$, so $\left\{y_{1}, \ldots, y_{d}\right\}$ is $\delta$-dependent modulo $\mathcal{I}_{1}$. Thus $\operatorname{dim} \mathcal{I}_{1} \leq d-1$. Now we claim $y_{1}, \ldots, y_{d-1}$ are $\delta$-independent modulo $\mathcal{I}_{1}$, which proves $\operatorname{dim} \mathcal{I}_{1}=d-1$. Suppose to the contrary that $y_{1}, \ldots, y_{d-1}$ are $\delta$-dependent modulo $\mathcal{I}_{1}$. Thus there exists a nonzero $\delta$-polynomial $p\left(y_{1}, \ldots, y_{d-1}\right) \in \mathcal{I}_{1}$. Take $p \in \mathcal{F}\left\{\widetilde{\mathbf{u}}_{f}, y_{1}, \ldots, y_{d-1}, u_{0}\right\}$. Then

$$
p\left(\widetilde{\mathbf{u}}_{f}, \xi_{1}, \ldots, \xi_{d-1},-f_{0}(\xi)\right)=0 .
$$

That is, $\xi_{1}, \ldots, \xi_{d-1},-f_{0}(\xi)$ are $\delta$-dependent over $\mathcal{F}\left\langle\widetilde{\mathbf{u}}_{f}\right\rangle$. Now we specialize $u_{d 0}$ to -1 , and the other $u \in \widetilde{\mathbf{u}}_{f}$ to zero, where $u_{d 0}$ refers to the coefficient of the monomial $y_{d}$ in $f$. Then $-f_{0}(\xi)$ is specialized to $\xi_{d}$. By Theorem 2.16, $\xi_{1}, \ldots, \xi_{d}$ are $\delta$-dependent over $\mathcal{F}$, which is a contradiction. So in this case $\operatorname{dim} \mathcal{I}_{1}=d-1$. 
Now, it remains to show the case $d=0$. Since $d=0$, by Lemma 3.5. $\mathcal{I}_{0} \cap$ $\mathcal{F}\left\langle\widetilde{\mathbf{u}}_{f}\right\rangle\left\{u_{0}\right\} \neq\{0\}$. So $\mathcal{I}_{0} \cap \mathcal{F}\left\langle\mathbf{u}_{f}\right\rangle \neq\{0\}$, and consequently $\mathcal{I}_{1}=\left[\mathcal{I}_{0}\right]$ in $\mathcal{F}\left\langle\mathbf{u}_{f}\right\rangle\{\mathbb{Y}\}$ is the unit ideal.

A special case of Theorem 3.6 is particularly interesting, and its algebraic counterpart is often listed as a theorem in algebraic geometry textbooks [16, p.54, p.110].

Theorem 3.7. Let $\mathcal{I}$ be a prime $\delta$-ideal in $\mathcal{F}\{\mathbb{Y}\}$ with dimension $d>0$. Let $\left\{u_{0}, u_{1}, \ldots, u_{n}\right\} \subset \mathcal{E}$ be a set of $\delta$ - $\mathcal{F}$-indeterminates. Then $\mathcal{I}_{1}=\left[\mathcal{I}, u_{0}+u_{1} y_{1}+\right.$ $\left.\cdots+u_{n} y_{n}\right]$ is a prime $\delta$-ideal in $\mathcal{F}\left\langle u_{0}, u_{1}, \ldots, u_{n}\right\rangle\{\mathbb{Y}\}$ with dimension $d-1$.

Theorem 3.6 is also valid for a wider class of $\delta$-polynomials. A $\delta$-polynomial $f$ in $\mathbb{Y}$ is said to be quasi-generic if 1 ) the coefficients of $f$ as a $\delta$-polynomial in $y_{1}, \ldots, y_{n}$ are $\delta$-indeterminates and 2) in addition to the degree zero term, for each $1 \leq i \leq n, f$ also contains at least one $\delta$-monomial in $\mathcal{F}\left\{y_{i}\right\} \backslash \mathcal{F}$. For instance, $f=u_{0}+u_{1} y_{1}+u_{2} y_{1} y_{2}$ is not quasi-generic, because $f$ contains no monomials in $\mathcal{F}\left\{y_{2}\right\} \backslash \mathcal{F}$.

The proof for Theorem 3.6 can be easily adapted to prove the following result.

Corollary 3.8. Let $\mathcal{I}$ be a prime $\delta$-ideal in $\mathcal{F}\{\mathbb{Y}\}$ with dimension $d$ and $f$ a quasigeneric $\delta$-polynomial with $\mathbf{u}_{f}$ as the set of coefficients. If $d>0$, then $\mathcal{I}_{1}=[\mathcal{I}, f]$ is a prime $\delta$-ideal in $\mathcal{F}\left\langle\mathbf{u}_{f}\right\rangle\{\mathbb{Y}\}$ with dimension $d-1$. Also, if $d=0$, then $\mathcal{I}_{1}$ is the unit ideal in $\mathcal{F}\left\langle\mathbf{u}_{f}\right\rangle\{\mathbb{Y}\}$.

By independent generic (resp. quasi-generic) $\delta$-polynomials, we mean that the coefficients of all of them are $\delta$-independent over $\mathcal{F}$. As a direct consequence, we can show that the dimension conjecture is valid for quasi-generic $\delta$-polynomials.

Theorem 3.9 (Generic Dimension Theorem). Let $f_{1}, \ldots, f_{r}$ be independent quasigeneric $\delta$-polynomials in $\mathcal{F}\langle\mathbf{u}\rangle\{\mathbb{Y}\}$ with $r \leq n$ and $\mathbf{u}$ the set of coefficients of all $f_{i}$. Then $\left[f_{1}, \ldots, f_{r}\right] \subset \mathcal{F}\langle\mathbf{u}\rangle\{\mathbb{Y}\}$ is a prime $\delta$-ideal with dimension $n-r$. Also, if $r>n,\left[f_{1}, \ldots, f_{r}\right]$ is the unit ideal.

Proof. We prove the theorem by induction. Let $\mathcal{I}=[0]$. When $r=1$, by Corollary 3.8. $\left[f_{1}\right]$ is prime with dimension $n-1$. Assuming this holds for $r-1$, now consider the case $r \leq n$. By the hypothesis, $\left[f_{1}, \ldots, f_{r-1}\right]$ is a prime $\delta$-ideal with dimension $n-r+1$. Note that the coefficients of $f_{r}$ are $\delta$-indeterminates over $\mathcal{F}\left\langle\mathbf{u}_{f_{1}}, \ldots, \mathbf{u}_{f_{r-1}}\right\rangle$. Using Corollary 3.8 again, $\left[f_{1}, \ldots, f_{r}\right]$ is a prime $\delta$-ideal with dimension $n-r$. When $r>n$, since $\left[f_{1}, \ldots, f_{n}\right]$ is of dimension zero, by Corollary $3.8\left[f_{1}, \ldots, f_{r}\right]$ is the unit ideal.

3.2. Order of a system of generic differential polynomials. In this section, we consider the order of the intersection of a $\delta$-variety by a generic $\delta$-hypersurface. Before proving the main result, we give a series of lemmas and theorems.

Lemma 3.10. Let $\mathcal{I}$ be a prime $\delta$-ideal in $\mathcal{F}\{\mathbb{Y}\}$ with dimension $n-1$. Suppose $\{f\}$ is a characteristic set of $\mathcal{I}$ w.r.t. some ranking $\mathscr{R}$ and $f$ is irreducible. Then for any other ranking $\overline{\mathscr{R}},\{f\}$ is also a characteristic set of $\mathcal{I}$.

Proof. Denote $f$ to be $\bar{f}$ under the ranking $\overline{\mathscr{R}}$. By Theorem $2.2, \mathcal{I}=\operatorname{sat}(f)$ and $\overline{\mathcal{I}}=\operatorname{sat}(\bar{f})$ are prime $\delta$-ideals with $f$ and $\bar{f}$ as characteristic sets, respectively. We need to show that $\mathcal{I}=\overline{\mathcal{I}}$. Let $S$ be the separant of $f$. Then for $g \in \operatorname{sat}(f)$, we have 
$S^{m} g=h f+h_{1} f^{\prime}+\cdots+h_{s} f^{(s)}$ for $m, s \in \mathbb{N}$. Then, $S^{m} g \in \operatorname{sat}(\bar{f})$. Since $\operatorname{sat}(\bar{f})$ is prime, we need only show that $S$ is not in sat $(\bar{f})$. Suppose the contrary, $S \in \operatorname{sat}(\bar{f})$. Since $S$ is partially reduced w.r.t. $\bar{f}$, we have $S=h \bar{f}$ for a $\delta$-polynomial $h$, which is impossible since $S=\frac{\partial f}{\partial u_{f}}$. So $\mathcal{I} \subseteq \overline{\mathcal{I}}$. Similarly, we can prove that $\mathcal{I} \supseteq \overline{\mathcal{I}}$, thus $\mathcal{I}=\overline{\mathcal{I}}$

If $\mathcal{S}$ is any set of $\delta$-polynomials in $\mathcal{E}\{\mathbb{Y}\}$, then its set of zeros in $\mathcal{E}^{n}$ is called the $\delta$-variety of $\mathcal{S}$, still denoted by $\mathbb{V}(\mathcal{S})$. The following lemma generalizes a result in [8, p.5] to the case of positive dimensions.

Lemma 3.11. Let the $\delta$-variety of a system $\mathcal{S}$ of $\delta$-polynomials in $\mathcal{F}\{\mathbb{Y}\}$ have a component $V$ of dimension $d$ and order $h$. Let $\overline{\mathcal{S}}$ be obtained from $\mathcal{S}$ by replacing $y_{1}^{(k)}$ by $y_{1}^{(k+1)}(k=0,1, \ldots)$ in all of the $\delta$-polynomials of $\mathcal{S}$. Then the $\delta$-variety of $\overline{\mathcal{S}}$ has a component $\bar{V}$ of dimension $d$ and order $h_{1}$ such that $h \leq h_{1} \leq h+1$. Moreover, if there exists a parametric set $\mathbb{U}$ not containing $y_{1}$ such that the relative order of $\mathbb{I}(V)$ w.r.t. $\mathbb{U}$ is $h$, then the order of $\bar{V}$ is $h+1$; otherwise, the order of $\bar{V}$ is $h$. In particular, if $d=0$, then $\operatorname{ord}(\bar{V})=h+1$.

Proof. Let $\left(\xi_{1}, \ldots, \xi_{n}\right)$ be a generic point of $V$ and $\mathcal{I}=\mathbb{I}(V) \in \mathcal{F}\{\mathbb{Y}\}$. It is clear that $\left[z^{\prime}-\xi_{1}\right]$ is a prime $\delta$-ideal in $\mathcal{F}\left\langle\xi_{1}, \ldots, \xi_{n}\right\rangle\{z\}$. Let $\eta$ be a generic point of $\left[z^{\prime}-\xi_{1}\right]$. Then $\left(\eta, \xi_{2}, \ldots, \xi_{n}\right)$ is a point of $\overline{\mathcal{S}}$. Suppose this point lies in a component $\bar{V}$ of $\overline{\mathcal{S}}$, which has a generic point $\left(\eta_{1}, \ldots, \eta_{n}\right)$. Then $\left(\eta_{1}, \eta_{2}, \ldots, \eta_{n}\right)$ is specialized to $\left(\eta, \xi_{2}, \ldots, \xi_{n}\right)$, and correspondingly $\left(\eta_{1}^{\prime}, \eta_{2}, \ldots, \eta_{n}\right)$ is specialized to $\left(\xi_{1}, \xi_{2}, \ldots, \xi_{n}\right)$. Since $\left(\xi_{1}, \ldots, \xi_{n}\right)$ is a generic point of $V$ and $\left(\eta_{1}^{\prime}, \eta_{2}, \ldots, \eta_{n}\right)$ is a zero of $\mathcal{S}$, the latter specialization is generic, that is, $\left(\eta_{1}^{\prime}, \eta_{2}, \ldots, \eta_{n}\right)$ is a generic point of $V$. We claim that any parametric set $\mathbb{U}$ of $\mathcal{I}$ is a parametric set of $\mathbb{I}(\bar{V})$, and $\operatorname{ord}_{\mathbb{U}} \mathcal{I} \leq \operatorname{ord}_{\mathbb{U}} \mathbb{I}(\bar{V}) \leq \operatorname{ord}_{\mathbb{U}} \mathcal{I}+1$, which follows that $\operatorname{dim}(\bar{V})=d$, and by Theorem 2.11, $h \leq \operatorname{ord}(\bar{V}) \leq h+1$. Let $\mathbb{U}$ be any parametric set of $\mathcal{I}$. We consider the following two cases.

Case 1: $y_{1} \notin \mathbb{U}$. Suppose $\mathbb{U}$ is the set of $y_{2}, \ldots, y_{d+1}$. By Corollary 2.8 we have

$$
\operatorname{ord}_{y_{2}, \ldots, y_{d+1}} \mathcal{I}=\operatorname{tr} . \operatorname{deg} \mathcal{F}\left\langle\xi_{1}, \ldots, \xi_{d}, \xi_{d+1}, \ldots, \xi_{n}\right\rangle / \mathcal{F}\left\langle\xi_{2}, \ldots, \xi_{d+1}\right\rangle .
$$

Since $\xi_{2}, \ldots, \xi_{d+1}$ are $\delta$-independent over $\mathcal{F}, \eta_{2}, \ldots, \eta_{d+1}$ must be $\delta$-independent over $\mathcal{F}$, i.e. $\mathbb{I}(\bar{V}) \cap \mathcal{F}\{\mathbb{U}\}=\{0\}$.

$$
\begin{aligned}
& \operatorname{tr.deg} \mathcal{F}\left\langle\eta_{1}, \eta_{2}, \ldots, \eta_{n}\right\rangle / \mathcal{F}\left\langle\eta_{2}, \ldots, \eta_{d+1}\right\rangle \\
\geq \quad & \operatorname{tr.deg} \mathcal{F}\left\langle\eta, \xi_{2}, \ldots, \xi_{n}\right\rangle / \mathcal{F}\left\langle\xi_{2}, \ldots, \xi_{d+1}\right\rangle \\
& \quad\left(\text { for }\left(\eta_{1}, \eta_{2}, \ldots, \eta_{n}\right) \text { can be specialized to }\left(\eta, \xi_{2}, \ldots, \xi_{n}\right)\right) \\
= & \operatorname{tr.deg} \mathcal{F}\left\langle\xi_{1}, \ldots, \xi_{n}\right\rangle / \mathcal{F}\left\langle\xi_{2}, \ldots, \xi_{d+1}\right\rangle+\operatorname{tr} . \operatorname{deg} \mathcal{F}\left\langle\xi_{1}, \ldots, \xi_{n}\right\rangle\langle\eta\rangle / \mathcal{F}\left\langle\xi_{1}, \ldots, \xi_{n}\right\rangle \\
= & \operatorname{ord}_{y_{2}, \ldots, y_{d+1}} \mathcal{I}+1
\end{aligned}
$$

and

$$
\begin{aligned}
& \operatorname{tr} . \operatorname{deg} \mathcal{F}\left\langle\eta_{1}, \ldots, \eta_{n}\right\rangle / \mathcal{F}\left\langle\eta_{2}, \ldots, \eta_{d+1}\right\rangle \\
\leq & 1+\operatorname{tr} \cdot \operatorname{deg} \mathcal{F}\left\langle\eta_{1}^{\prime}, \eta_{2} \ldots, \eta_{n}\right\rangle / \mathcal{F}\left\langle\eta_{2}, \ldots, \eta_{d+1}\right\rangle \\
= & 1+\operatorname{ord}_{y_{2}, \ldots, y_{d+1}} \mathcal{I},
\end{aligned}
$$

so tr.deg $\mathcal{F}\left\langle\eta_{1}, \ldots, \eta_{n}\right\rangle / \mathcal{F}\left\langle\eta_{2}, \ldots, \eta_{d+1}\right\rangle=1+\operatorname{ord}_{y_{2}, \ldots, y_{d+1}} \mathcal{I}<\infty$. Thus $\bar{V}$ is of dimension $d$ and $\left\{y_{2}, \ldots, y_{d+1}\right\}$ is a parametric set of $\mathbb{I}(\bar{V})$. Moreover, the relative order of $\mathbb{I}(\bar{V})$ w.r.t. $y_{2}, \ldots, y_{d+1}$ is $\operatorname{ord}_{y_{2}, \ldots, y_{d+1}} \mathcal{I}+1$. 
Case 2: $y_{1} \in \mathbb{U}$. Suppose $\mathbb{U}=\left\{y_{1}, \ldots, y_{d}\right\}$. Then by Corollary 2.8, $\operatorname{ord}_{\mathbb{U}} \mathcal{I}=$ $\operatorname{ord}_{y_{1}, \ldots, y_{d}} \mathcal{I}=\operatorname{tr} . \operatorname{deg} \mathcal{F}\left\langle\xi_{1}, \ldots, \xi_{n}\right\rangle / \mathcal{F}\left\langle\xi_{1}, \ldots, \xi_{d}\right\rangle$. We have seen that $\left(\eta_{1}^{\prime}, \eta_{2}, \ldots, \eta_{n}\right)$ is a generic point of $V$. Since tr.deg $\mathcal{F}\left\langle\xi_{1}, \ldots, \xi_{n}\right\rangle\langle\eta\rangle / \mathcal{F}\left\langle\xi_{1}, \ldots, \xi_{n}\right\rangle=1$ and $\left(\eta_{1}, \eta_{2}\right.$, $\left.\ldots, \eta_{n}\right)$ can be specialized to $\left(\eta, \xi_{2}, \ldots, \xi_{n}\right), \eta_{1}$ is algebraically independent over $\mathcal{F}\left\langle\eta_{1}^{\prime}, \eta_{2}, \ldots, \eta_{n}\right\rangle$. So

$$
\begin{array}{ll} 
& \operatorname{tr} \cdot \operatorname{deg} \mathcal{F}\left\langle\eta_{1}, \ldots, \eta_{n}\right\rangle / \mathcal{F}\left\langle\eta_{1}, \ldots, \eta_{d}\right\rangle \\
= & \operatorname{tr} \cdot \operatorname{deg} \mathcal{F}\left(\eta_{1}\right)\left\langle\eta_{1}^{\prime}, \eta_{2}, \ldots, \eta_{n}\right\rangle / \mathcal{F}\left(\eta_{1}\right)\left\langle\eta_{1}^{\prime}, \eta_{2}, \ldots, \eta_{d}\right\rangle \\
= & \operatorname{tr} \cdot \operatorname{deg} \mathcal{F}\left\langle\eta_{1}^{\prime}, \eta_{2}, \ldots, \eta_{n}\right\rangle / \mathcal{F}\left\langle\eta_{1}^{\prime}, \eta_{2}, \ldots, \eta_{d}\right\rangle \\
& \quad\left(\text { for tr.deg } \mathcal{F}\left\langle\eta_{1}^{\prime}, \ldots, \eta_{n}\right\rangle\left(\eta_{1}\right) / \mathcal{F}\left\langle\eta_{1}^{\prime}, \ldots, \eta_{n}\right\rangle=1\right) \\
= & \operatorname{ord}_{y_{1}, \ldots, y_{d}} \mathcal{I} .
\end{array}
$$

Since $\left(\eta_{1}, \ldots, \eta_{d}\right)$ can be specialized to $\left(\eta, \xi_{2}, \ldots, \xi_{d}\right)$ over $\mathcal{F}, d \geq$ d.tr.deg $\mathcal{F}\left\langle\eta_{1}\right.$, $\left.\ldots, \eta_{d}\right\rangle / \mathcal{F} \geq$ d.tr.deg $\mathcal{F}\left\langle\eta, \xi_{2}, \ldots, \xi_{d}\right\rangle / \mathcal{F} \geq$ d.tr.deg $\mathcal{F}\left\langle\xi_{1}, \xi_{2}, \ldots, \xi_{d}\right\rangle / \mathcal{F}=d$. Since tr.deg $\mathcal{F}\left\langle\eta_{1}, \ldots, \eta_{n}\right\rangle / \mathcal{F}\left\langle\eta_{1}, \ldots, \eta_{d}\right\rangle<\infty$, we have d.tr.deg $\mathcal{F}\left\langle\eta_{1}, \ldots, \eta_{d}, \ldots, \eta_{n}\right\rangle / \mathcal{F}=$ $d$. Thus in this case, $\operatorname{dim}(\bar{V})=d$, and $\mathbb{U}=\left\{y_{1}, \ldots, y_{d}\right\}$ is a parametric set of $\mathbb{I}(\bar{V})$ with $\operatorname{ord}_{y_{1}, \ldots, y_{d}} \mathbb{I}(\bar{V})=\operatorname{ord}_{y_{1}, \ldots, y_{d}} \mathcal{I}$.

Considering the two cases together, we can see $\operatorname{dim}(\bar{V})=d$. Also, by Theorem 2.11, $h \leq \operatorname{ord}(\bar{V}) \leq h+1$. Moreover, if there exists a parametric set $\mathbb{U}$ not containing $y_{1}$ such that the relative order of $\mathbb{I}(V)$ w.r.t. $\mathbb{U}$ is $h$, then the order of $\bar{V}$ is $h+1$; otherwise, the order of $\bar{V}$ is $h$. In particular, if $d=0$, then $y_{1} \notin \mathbb{U}=\emptyset$. From Case 1, $\operatorname{ord}(\bar{V})=\operatorname{ord}(V)+1=h+1$.

Let $\mathcal{G} \subset \mathcal{E}$ be a $\delta$-extension field of $\mathcal{F}$. By a $\delta$ - $\mathcal{F}$-isomorphism of $\mathcal{G}$, we mean a $\delta$-isomorphic mapping of $\mathcal{G}$ onto a $\delta$-field $\mathcal{G}^{\prime} \subset \mathcal{E}$ such that (a) $\mathcal{G}^{\prime}$ is an extension of $\mathcal{F}$, (b) the $\delta$-isomorphic mapping leaves each element of $\mathcal{F}$ invariant. By means of well-ordering methods, it is easy to show that a $\delta$ - $\mathcal{F}$-isomorphism of $\mathcal{G}$ can be extended to a $\delta$ - $\mathcal{F}$-automorphism of $\mathcal{E}$. We will use the following result regarding $\delta$-isomorphisms.

Theorem 3.12 ([20]). Let $\mathcal{G} \subset \mathcal{E}$ be a $\delta$-extension field of $\mathcal{F}$ and $\gamma \in \mathcal{G}$. A necessary and sufficient condition that $\gamma$ be a primitive element of $\mathcal{G}$, i.e. $\mathcal{G}=\mathcal{F}\langle\gamma\rangle$, is that no $\mathcal{F}$-isomorphism of $\mathcal{G}$ other than the identity leaves $\gamma$ invariant.

The following theorem as well as Theorem 3.6 prove Theorem 1.1

Theorem 3.13. Let $\mathcal{I}$ be a prime $\delta$-F -ideal with dimension $d>0$ and order $h$, and $f$ a generic $\delta$-polynomial of order s. Then $\mathcal{I}_{1}=[\mathcal{I}, f]$ is a prime $\delta$-ideal in $\mathcal{F}\left\langle\mathbf{u}_{f}\right\rangle\{\mathbb{Y}\}$ with dimension $d-1$ and order $h+s$.

Proof. By Theorem 3.6. $\mathcal{I}_{1}$ is prime with dimension $d-1$. Now we prove the order of $\mathcal{I}_{1}$ is $h+s$.

Let $\mathcal{A}$ be a characteristic set of $\mathcal{I}$ w.r.t. an orderly ranking $\mathscr{R}$ with $y_{1}, \ldots, y_{d}$ as a parametric set. By Theorem 2.6. $\operatorname{ord}(\mathcal{A})=h$. Suppose $\xi=\left(\xi_{1}, \ldots, \xi_{n}\right)$ is a generic point of $\mathcal{I}$ that is free from the pure extension field $\mathcal{F}\left\langle\mathbf{u}_{f}\right\rangle$ over $\mathcal{F}$. Let $f=u_{0}+f_{0}$, where $f_{0}=\sum_{\operatorname{deg}(m) \geq 1} u_{m} m$. Let $\mathcal{I}_{0}=[\mathcal{I}, f]$ in $\mathcal{F}\left\langle\widetilde{\mathbf{u}}_{f}\right\rangle\left\{y_{1}, \ldots, y_{n}, u_{0}\right\}$, where $\widetilde{\mathbf{u}}_{f}$ is defined in (3.1). By Lemma 3.5 $\mathcal{I}_{0}$ is a prime $\delta$-ideal of dimension $d$ 
with a generic zero $\left(\xi_{1}, \ldots, \xi_{n},-f_{0}(\xi)\right)$, and $u_{0}$ is $\delta$-independent modulo $\mathcal{I}_{0} . \mathcal{I}_{0}$ and $\mathcal{I}_{1}$ have such relations: Any characteristic set of $\mathcal{I}_{0}$ with $u_{0}$ in the parametric set is a characteristic set of $\mathcal{I}_{1}$, and conversely, any characteristic set of $\mathcal{I}_{1}$, by clearing denominators in $\mathcal{F}\left\langle\widetilde{\mathbf{u}}_{f}\right\rangle\left\{u_{0}\right\}$ when necessary, is a characteristic set of $\mathcal{I}_{0}$ with $u_{0}$ in the parametric set. By Theorem 2.11, we have $\operatorname{ord}\left(\mathcal{I}_{1}\right) \leq \operatorname{ord}\left(\mathcal{I}_{0}\right)$.

We claim that $\operatorname{ord}\left(\mathcal{I}_{0}\right) \leq h+s$. As a consequence, $\operatorname{ord}\left(\mathcal{I}_{1}\right) \leq h+s$. To prove this claim, let $\mathcal{I}_{0}^{(i)}=\left[\mathcal{I}, u_{0}^{(i)}+f_{0}\right](i=0, \ldots, s)$ in $\mathcal{F}\left\langle\widetilde{\mathbf{u}}_{f}\right\rangle\left\{y_{1}, \ldots, y_{n}, u_{0}\right\}$. Similar to the proof of Lemma 3.5. $\mathcal{I}_{0}^{(i)}$ is a prime $\delta$-ideal of dimension $d$. Let $\bar{f}$ be the pseudo remainder of $u_{0}^{(s)}+f_{0}$ w.r.t. $\mathcal{A}$ under the ranking $\mathscr{R}$. Clearly, $\operatorname{ord}\left(\bar{f}, u_{0}\right)=s$. It is obvious that for some orderly ranking, $\{\mathcal{A}, \bar{f}\}$ is a characteristic set of $\mathcal{I}_{0}^{(s)}$ with $y_{1}, \ldots, y_{d}$ as a parametric set. So $\operatorname{ord}\left(\mathcal{I}_{0}^{(s)}\right)=h+s$. Using Lemma $3.11 s$ times, we have $\operatorname{ord}\left(\mathcal{I}_{0}\right) \leq \operatorname{ord}\left(\mathcal{I}_{0}^{(1)}\right) \leq \cdots \leq \operatorname{ord}\left(\mathcal{I}_{0}^{(s)}\right)=h+s$.

Now, it suffices to show $\operatorname{ord}\left(\mathcal{I}_{1}\right) \geq h+s$. Let $w=u_{0}+\sum_{i=1}^{d} \sum_{j=0}^{s} u_{i j} y_{i}^{(j)}$ be a new $\delta$-indeterminate. Let $\mathbf{u}_{g}$ be the set of coefficients of $g=w+\sum_{i=d+1}^{n} \sum_{j=0}^{s} u_{i j} y_{i}^{(j)}+$ $f_{1}$ regarded as a $\delta$-polynomial in $w$ and $\mathbb{Y}$, where $f_{1}$ is the nonlinear part of $f$ in $\mathbb{Y}$. We denote $\mathcal{F}_{1}=\mathcal{F}\left\langle\mathbf{u}_{g}\right\rangle$. Then $\mathcal{I}_{2}=[\mathcal{I}, g]$ in $\mathcal{F}_{1}\left\{y_{1}, \ldots, y_{n}, w\right\}$ is a prime $\delta$-ideal with a generic point $\left(\xi_{1}, \ldots, \xi_{n}, \gamma\right)$, where $\gamma=-\sum_{i=d+1}^{n} \sum_{j=0}^{s} u_{i j} \xi_{i}^{(j)}-$ $f_{1}\left(\xi_{1}, \ldots, \xi_{n}\right)$. We claim that $\gamma$ is a primitive element of $\mathcal{F}_{1}\left\langle\xi_{1}, \ldots, \xi_{d}\right\rangle\left\langle\xi_{d+1}, \ldots, \xi_{n}\right\rangle$ over $\mathcal{F}_{1}\left\langle\xi_{1}, \ldots, \xi_{d}\right\rangle$. By Theorem 3.12, it suffices to show that no $\mathcal{F}_{1}\left\langle\xi_{1}, \ldots, \xi_{d}\right\rangle$ isomorphism of $\mathcal{F}_{1}\left\langle\xi_{1}, \ldots, \xi_{d}\right\rangle\left\langle\xi_{d+1}, \ldots, \xi_{n}\right\rangle$ other than the identity leaves $\gamma$ invariant. Let $\varphi$ be any $\delta$ - $\mathcal{F}_{1}\left\langle\xi_{1}, \ldots, \xi_{d}\right\rangle$-isomorphism of $\mathcal{F}_{1}\left\langle\xi_{1}, \ldots, \xi_{d}\right\rangle\left\langle\xi_{d+1}, \ldots, \xi_{n}\right\rangle$ which leaves $\gamma$ invariant, and $\varphi\left(\xi_{d+i}\right)=\eta_{d+i}(i=1, \ldots, n-d)$. Since each $\xi_{d+i}(i=$ $1, \ldots, n-d)$ is $\delta$-algebraic over $\mathcal{F}\left\langle\xi_{1}, \ldots, \xi_{d}\right\rangle$ and $\varphi$ is an isomorphism leaving each element of $\mathcal{F}\left\langle\xi_{1}, \ldots, \xi_{d}\right\rangle$ invariant, we can see that each $\eta_{d+i}(i=1, \ldots, n-d)$ is also $\delta$-algebraic over $\mathcal{F}\left\langle\xi_{1}, \ldots, \xi_{d}\right\rangle$. So, $\eta_{d+i}(i=1, \ldots, n-d)$ are also free from $\mathcal{F}\left\langle\mathbf{u}_{f}\right\rangle$. From $\varphi(\gamma)=\gamma$, we have $-\sum_{i=d+1}^{n} \sum_{j=0}^{s} u_{i j} \eta_{i}^{(j)}-f_{1}\left(\xi_{1}, \ldots, \xi_{d}, \eta_{d+1}, \ldots, \eta_{n}\right)=$ $-\sum_{i=d+1}^{n} \sum_{j=0}^{s} u_{i j} \xi_{i}^{(j)}-f_{1}\left(\xi_{1}, \ldots, \xi_{d}, \xi_{d+1}, \ldots, \xi_{n}\right)$, which can be rewritten as

$$
\sum_{i=d+1}^{n} \sum_{j=0}^{s} u_{i j}\left(\xi_{i}^{(j)}-\eta_{i}^{(j)}\right)+f_{1}\left(\xi_{1}, \ldots, \xi_{n}\right)-f_{1}\left(\xi_{1}, \ldots, \xi_{d}, \eta_{d+1}, \ldots, \eta_{n}\right)=0
$$

Since $\mathbf{u}_{f}$ are $\delta$-indeterminates over $\mathcal{F}\left\langle\xi_{1}, \ldots, \xi_{n}, \eta_{d+1}, \ldots, \eta_{n}\right\rangle$, we have $\xi_{i}-\eta_{i}=$ $0(i=d+1, \ldots, n)$. So $\varphi$ must be the identity map and the claim follows.

Since $\mathcal{F}_{1}\left\langle\xi_{1}, \ldots, \xi_{d}, \xi_{d+1}, \ldots, \xi_{n}\right\rangle=\mathcal{F}_{1}\left\langle\xi_{1}, \ldots, \xi_{d}\right\rangle\langle\gamma\rangle, \gamma$ is $\delta$-algebraic over $\mathcal{F}_{1}\left\langle\xi_{1}\right.$, $\left.\ldots, \xi_{d}\right\rangle$ and each $\xi_{d+i} \in \mathcal{F}_{1}\left\langle\xi_{1}, \ldots, \xi_{d}\right\rangle\langle\gamma\rangle(i=1, \ldots, n-d)$. Let $R\left(\xi_{1}, \ldots, \xi_{d}, w\right)$ be an irreducible $\delta$-polynomial in $\mathcal{F}_{1}\left\langle\xi_{1}, \ldots, \xi_{d}\right\rangle\{w\}$ annulling $\gamma$ of the lowest order. By clearing denominators when necessary, suppose $R\left(y_{1}, \ldots, y_{d}, w\right)$ is an irreducible $\delta$-polynomial in $\mathcal{F}_{1}\left\{y_{1}, \ldots, y_{d}, w\right\}$. Clearly, $R\left(y_{1}, \ldots, y_{d}, w\right) \in \mathcal{I}_{2}$. Also, there exist $A_{i} \in \mathcal{I}_{2}$ with the form $A_{i}=P_{i}\left(y_{1}, \ldots, y_{d}, w\right) y_{d+i}+Q_{i}\left(y_{1}, \ldots, y_{d}, w\right)$ $(i=1, \ldots, n-d)$, which are reduced w.r.t. $R$. Since $\mathcal{I}_{2} \cap \mathcal{F}_{1}\left\{y_{1}, \ldots, y_{d}, w\right\}$ is a $d$-dimensional prime $\delta$-ideal, by Lemma 3.10, $\{R\}$ is its characteristic set w.r.t. any ranking. So for the elimination ranking $y_{1} \prec \cdots \prec y_{d} \prec w \prec y_{d+1} \prec \cdots \prec y_{n}$, a characteristic set of $\mathcal{I}_{2}$ is $\left\{R\left(y_{1}, \ldots, y_{d}, w\right), A_{1}, \ldots, A_{n-d}\right\}$. Since $\mathcal{F}_{1}\left\langle\xi_{1}, \ldots, \xi_{n}, \gamma\right\rangle=$ $\mathcal{F}_{1}\left\langle\xi_{1}, \ldots, \xi_{n}\right\rangle$, by Corollary 2.8, $\operatorname{ord}_{y_{1}, \ldots, y_{d}} \mathcal{I}_{2}=\operatorname{ord}_{y_{1}, \ldots, y_{d}}(\mathcal{I})=\operatorname{ord}(\mathcal{A})=h$. Thus, $\operatorname{ord}(R, w)=h$. 
Let $\mathbb{U}=\left\{u_{i j}: i=1, \ldots, d ; j=0, \ldots, s\right\} . \quad \operatorname{In} \mathcal{F}_{1}\langle\mathbb{U}\rangle\left\{w, y_{1}, \ldots, y_{n}\right\}, \mathcal{I}_{2}$ is also prime with $R\left(y_{1}, \ldots, y_{d}, w\right), A_{1}, \ldots, A_{n-d}$ as a characteristic set w.r.t. the elimination ranking $y_{1} \prec \cdots \prec y_{d} \prec w \prec y_{d+1} \prec \cdots \prec y_{n}$. Let

$$
\begin{aligned}
& \phi: \mathcal{F}_{1}\langle\mathbb{U}\rangle\left\{y_{1}, \ldots, y_{n}, w\right\} \quad \longrightarrow \quad \mathcal{F}_{1}\langle\mathbb{U}\rangle\left\{y_{1}, \ldots, y_{n}, u_{0}\right\}, \\
& w \\
& y_{i} \\
& u_{0}+\sum_{i=1}^{d} \sum_{y_{i}}^{s} u_{i j} y_{i}^{(j)} \text {, }
\end{aligned}
$$

be a $\delta$-homomorphism over $\mathcal{F}_{1}\langle\mathbb{U}\rangle$. Clearly, this is a $\delta$-isomorphism which maps $\mathcal{I}_{2}$ to $\mathcal{I}_{0}$. It is obvious that $\mathcal{I}_{0}$ has $\phi(R), \phi\left(A_{1}\right), \ldots, \phi\left(A_{n-d}\right)$ as a characteristic set w.r.t. the elimination ranking $y_{1} \prec \cdots \prec y_{d} \prec u_{0} \prec y_{d+1} \prec \cdots \prec y_{n}$ with $\operatorname{rk}\left(\phi\left(A_{i}\right)\right)=y_{d+i}(i=1, \ldots, n-d)$. We claim that $\operatorname{ord}\left(\phi(R), y_{1}\right) \geq h+s$. If $\operatorname{ord}\left(R, y_{1}\right) \geq h+s$, rewrite $R$ in the form $R=\sum_{\psi_{\nu}(w) \neq 1} p_{\nu}\left(y_{1}, \ldots, y_{d}\right) \psi_{\nu}(w)+$ $p\left(y_{1}, \ldots, y_{d}\right)$, where $\psi_{\nu}(w)$ are monomials in $w$ and its derivatives. Then

$$
\begin{aligned}
\phi(R)= & \sum_{\psi_{\nu} \neq 1} p_{\nu}\left(y_{1}, \ldots, y_{d}\right) \psi_{\nu}\left(u_{0}+\sum_{i=1}^{d} \sum_{j=0}^{s} u_{i j} y_{i}^{(j)}\right)+p\left(y_{1}, \ldots, y_{d}\right) \\
= & \sum_{\psi_{\nu} \neq 1} p_{\nu}\left(y_{1}, \ldots, y_{d}\right) \psi_{\nu}\left(u_{0}\right)+p\left(y_{1}, \ldots, y_{d}\right) \\
& + \text { terms involving } u_{i j}(i=1, \ldots, d ; j=0, \ldots, s) \text { and their derivatives. }
\end{aligned}
$$

Clearly, in this case we have ord $\left(\phi(R), y_{1}\right) \geq \max \left\{\operatorname{ord}\left(p_{\nu}, y_{1}\right), \operatorname{ord}\left(p, y_{1}\right)\right\}=\operatorname{ord}(R$, $\left.y_{1}\right) \geq h+s$. If $\operatorname{ord}\left(R, y_{1}\right)<h+s$, rewrite $R$ as a polynomial in $w^{(h)}$, that is, $R=$ $I_{l}\left(w^{(h)}\right)^{l}+I_{l-1}\left(w^{(h)}\right)^{l-1}+\cdots+I_{0}$. Then $\phi(R)=\phi\left(I_{l}\right)\left[\left(u_{0}+\sum_{i=1}^{d} \sum_{j=0}^{s} u_{i j} y_{i}^{(j)}\right)^{(h)}\right]^{l}+$ $\phi\left(I_{l-1}\right)\left[\left(u_{0}+\sum_{i=1}^{d} \sum_{j=0}^{s} u_{i j} y_{i}^{(j)}\right)^{(h)}\right]^{l-1}+\cdots+\phi\left(I_{0}\right)$. Since $\operatorname{ord}\left(\phi\left(I_{k}\right), y_{1}\right)<h+s$ $(k=0, \ldots, l)$, we have exactly ord $\left(\phi(R), y_{1}\right)=h+s$. Thus, consider the two cases together, $\operatorname{ord}\left(\phi(R), y_{1}\right) \geq h+s$.

Since $\mathcal{I}_{0} \cap \mathcal{F}_{1}\langle\mathbb{U}\rangle\left\{y_{1}, \ldots, y_{d}, u_{0}\right\}$ is a $d$-dimensional prime $\delta$-ideal, by Lemma 3.10 . $\{\phi(R)\}$ is its characteristic set w.r.t. any ranking, in particular, for the elimination ranking $u_{0} \prec y_{2} \prec \cdots \prec y_{d} \prec y_{1}$. So w.r.t. the elimination ranking $u_{0} \prec y_{2} \prec \cdots \prec$ $y_{d} \prec y_{1} \prec y_{d+1} \prec \cdots \prec y_{n},\left\{\phi(R), \phi\left(A_{1}\right), \ldots, \phi\left(A_{n-d}\right)\right\}$ is a characteristic set of $\mathcal{I}_{0}$, thus a characteristic set of $\mathcal{I}_{1}$. By Theorem 2.11 $\operatorname{ord}\left(\mathcal{I}_{1}\right) \geq \operatorname{ord}_{y_{2}, \ldots, y_{d}} \mathcal{I}_{1} \geq h+s$.

Thus, the order of $\mathcal{I}_{1}$ is $h+s$.

As a consequence, Theorem 3.7 can be strengthened as follows.

Theorem 3.14. Let $\mathcal{I}$ be a prime $\delta$-ideal in $\mathcal{F}\{\mathbb{Y}\}$ with dimension $d>0$ and order $h$. Let $\left\{u_{0}, u_{1}, \ldots, u_{n}\right\} \subset \mathcal{E}$ be a set of $\delta$ - $\mathcal{F}$-indeterminates. Then $\mathcal{I}_{1}=$ $\left[\mathcal{I}, u_{0}+u_{1} y_{1}+\cdots+u_{n} y_{n}\right]$ is a prime $\delta$-ideal in $\mathcal{F}\left\langle u_{0}, u_{1}, \ldots, u_{n}\right\rangle\{\mathbb{Y}\}$ with dimension $d-1$ and order $h$.

As another consequence, the dimension theorem for generic $\delta$-polynomials can be strengthened as follows.

Theorem 3.15. Let $f_{1}, \ldots, f_{r}(r \leq n)$ be independent generic $\delta$-polynomials with each $f_{i}$ of order $s_{i}$. Then $\left[f_{1}, \ldots, f_{r}\right]$ is a prime $\delta$-ideal with dimension $n-r$ and $\operatorname{order} \sum_{i=1}^{r} s_{i}$ over $\mathcal{F}\left\langle\mathbf{u}_{f_{1}}, \ldots, \mathbf{u}_{f_{r}}\right\rangle$.

Proof. We will prove the theorem by induction on $r$. Let $\mathcal{I}=[0] \subset \mathcal{F}\{\mathbb{Y}\}$. Clearly, $\mathcal{I}$ is a prime $\delta$-ideal of dimension $n$ and order 0 . For $r=1$, by Theorem 3.13 , $\left[f_{1}\right]=\left[\mathcal{I}, f_{1}\right]$ is a prime $\delta$-ideal of dimension $n-1$ and order $s_{1}$. So the assertion 
holds for $r=1$. Now suppose the assertion holds for $r-1$; we now prove it for $r$. By the hypothesis, $\mathcal{I}_{r-1}=\left[f_{1}, \ldots, f_{r-1}\right]$ is a prime $\delta$-ideal of dimension $n-r+1$ and order $\sum_{i=1}^{r-1} s_{i}$ over $\mathcal{F}\left\langle\mathbf{u}_{f_{1}}, \ldots, \mathbf{u}_{f_{r-1}}\right\rangle$. Since $f_{1}, \ldots, f_{r}$ are independent generic $\delta$-polynomials, using Theorem 3.13 again, $\mathcal{I}_{r}=\left[f_{1}, \ldots, f_{r}\right]$ is a prime $\delta$-ideal of dimension $n-r$ and order $\sum_{i=1}^{r} s_{i}$ over $\mathcal{F}\left\langle\mathbf{u}_{f_{1}}, \ldots, \mathbf{u}_{f_{r}}\right\rangle$. Thus, the theorem is proved.

Remark 3.16. When $f$ is a quasi-generic $\delta$-polynomial, Theorem 3.13 may not be true. A counterexample is as follows. Let $\mathcal{I}=\left[y_{2}, \ldots, y_{n}\right] \in \mathcal{F}\{\mathbb{Y}\}$ and $f=$ $u_{0}+u_{1} y_{1}+u_{2} y_{2}^{\prime \prime}+\cdots+u_{n} y_{n}^{\prime \prime}$. Clearly, $f$ is a quasi-generic $\delta$-polynomial and $[\mathcal{I}, f]$ is a prime $\delta$-ideal of dimension 0 . But $\operatorname{ord}([\mathcal{I}, f])=\operatorname{ord}(\mathcal{I})=0 \neq \operatorname{ord}(\mathcal{I})+\operatorname{ord}(f)=2$.

\section{Chow FORM FOR AN IRREDUCIBLE DifFERENTIAL VARIETy}

In this section, we define the differential Chow form and establish its properties by proving Theorem 1.2

4.1. Definition of the differential Chow form. Throughout this section, we assume that $V \subset \mathcal{E}^{n}$ is an irreducible $\delta$ - $\mathcal{F}$-variety with dimension $d$ and $\mathcal{I}=\mathbb{I}(V) \subset$ $\mathcal{F}\{\mathbb{Y}\}$. Let

$$
\widehat{\mathbf{u}}=\left\{u_{i j}(i=0, \ldots, d ; j=0, \ldots, n)\right\}
$$

be $(d+1)(n+1) \delta$ - $\mathcal{F}$-indeterminates in $\mathcal{E}$.

By saying that a point $\eta \in \mathcal{E}^{n}$ is free from the pure $\delta$-extension field $\mathcal{F}\langle\widehat{\mathbf{u}}\rangle$ over $\mathcal{F}$, we mean $\widehat{\mathbf{u}}$ are $\delta$-indeterminates over $\mathcal{F}\langle\eta\rangle$. Let $\xi=\left(\xi_{1}, \ldots, \xi_{n}\right)$ be a generic point of $V$ which is free from $\mathcal{F}\langle\widehat{\mathbf{u}}\rangle$ and $\zeta_{0}, \zeta_{1}, \ldots, \zeta_{d}$ elements of $\mathcal{F}\left\langle\mathbf{u}, \xi_{1}, \ldots, \xi_{n}\right\rangle$ :

$$
\zeta_{\sigma}=-\sum_{\rho=1}^{n} u_{\sigma \rho} \xi_{\rho}(\sigma=0, \ldots, d),
$$

where

$$
\mathbf{u}=\left\{u_{i j}(i=0, \ldots, d ; j=1, \ldots, n)\right\} .
$$

We will show that the $\delta$-transcendence degree of $\zeta_{0}, \ldots, \zeta_{d}$ over $\mathcal{F}\langle\mathbf{u}\rangle$ is $d$.

Lemma 4.1. d.tr.deg $\mathcal{F}\langle\mathbf{u}\rangle\left\langle\zeta_{0}, \ldots, \zeta_{d}\right\rangle / \mathcal{F}\langle\mathbf{u}\rangle=d$. Furthermore, if $d>0, \zeta_{1}, \ldots, \zeta_{d}$ are $\delta$-independent over $\mathcal{F}\langle\mathbf{u}\rangle$.

Proof. By Lemma 2.12, d.tr.deg $\mathcal{F}\langle\mathbf{u}\rangle\left\langle\xi_{1}, \ldots, \xi_{n}\right\rangle / \mathcal{F}\langle\mathbf{u}\rangle=d$. Since the $d+1$ elements $\zeta_{0}, \ldots, \zeta_{d}$ belong to $\mathcal{F}\left\langle\mathbf{u}, \xi_{1}, \ldots, \xi_{n}\right\rangle$, they are $\delta$-dependent over $\mathcal{F}\langle\mathbf{u}\rangle$. Then, we have d.tr.deg $\mathcal{F}\langle\mathbf{u}\rangle\left\langle\zeta_{0}, \ldots, \zeta_{d}\right\rangle / \mathcal{F}\langle\mathbf{u}\rangle \leq d$. Thus, if $d=0$, we have

$$
\text { d.tr.deg } \mathcal{F}\langle\mathbf{u}\rangle\left\langle\zeta_{0}, \ldots, \zeta_{d}\right\rangle / \mathcal{F}\langle\mathbf{u}\rangle=0 \text {. }
$$

Now, suppose $d>0$. We claim that $\zeta_{1}, \ldots, \zeta_{d}$ are $\delta$-independent over $\mathcal{F}\langle\mathbf{u}\rangle$, thus it follows that d.tr.deg $\mathcal{F}\langle\mathbf{u}\rangle\left\langle\zeta_{0}, \ldots, \zeta_{d}\right\rangle / \mathcal{F}\langle\mathbf{u}\rangle=d$. Suppose the contrary. Since $\zeta_{i} \in \mathcal{F}\left\{\mathbf{u}, \xi_{1}, \ldots, \xi_{n}\right\}$, when we specialize $u_{i j}$ to $-\delta_{k_{i} j}\left(j=1, \ldots, n, k_{i} \in\{1, \ldots, n\}\right)$, $\zeta_{i}$ will be specialized to $\xi_{k_{i}}$. Then from Theorem 2.16, we conclude that $\xi_{k_{1}}, \ldots, \xi_{k_{d}}$ are $\delta$-dependent over $\mathcal{F}$. Since we can choose $k_{1}, \ldots, k_{d}$ so that $\xi_{k_{1}}, \ldots, \xi_{k_{d}}$ are $\delta$ independent over $\mathcal{F}$, it amounts to a contradiction. Thus the claim is proved. 
Let $\mathbb{I}_{\zeta}$ be the prime $\delta$-ideal in the $\delta$-polynomial ring $\mathcal{R}=\mathcal{F}\langle\mathbf{u}\rangle\left\{z_{0}, \ldots, z_{d}\right\}$ having $\zeta=\left(\zeta_{0}, \ldots, \zeta_{d}\right)$ as a generic point. By Lemma 4.1, the dimension of $\mathbb{I}_{\zeta}$ is d. By Theorem [2.9, the characteristic set of $\mathbb{I}_{\zeta}$ w.r.t. any ranking consists of an irreducible $\delta$-polynomial $f\left(z_{0}, \ldots, z_{d}\right)$ in $\mathcal{R}$ and

$$
\mathbb{I}_{\zeta}=\operatorname{sat}(f) \text {. }
$$

Since the coefficients of $f\left(z_{0}, \ldots, z_{d}\right)$ are elements in $\mathcal{F}\langle\mathbf{u}\rangle$, without loss of generality, we assume $f\left(\mathbf{u} ; z_{0}, \ldots, z_{d}\right)$ is an irreducible $\delta$-polynomial in $\mathcal{F}\left\{\mathbf{u} ; z_{0}, \ldots, z_{d}\right\}$. We shall subsequently replace $\left\{z_{0}, \ldots, z_{d}\right\}$ by $\left\{u_{00}, \ldots, u_{d 0}\right\} \subset \widehat{\mathbf{u}}$, and obtain

$$
F\left(\mathbf{u}_{0}, \mathbf{u}_{1}, \ldots, \mathbf{u}_{d}\right)=f\left(\mathbf{u} ; u_{00}, \ldots, u_{d 0}\right) \in \mathcal{F}\{\widehat{\mathbf{u}}\},
$$

where $\widehat{\mathbf{u}}$ is from (4.1) and $\mathbf{u}_{i}=\left(u_{i 0}, \ldots, u_{i n}\right)$ for $i=0, \ldots, d$.

Definition 4.2 (Differential Chow form). The $\delta$-polynomial defined in (4.4) is called the differential Chow form of $V$ or the prime $\delta$-ideal $\mathcal{I}=\mathbb{I}(V)$.

A generic $\delta$-hyperplane is the zero set of $u_{0}+u_{1} y_{1}+\cdots+u_{n} y_{n}=0$, where $u_{i}$ are $\delta$ - $\mathcal{F}$-indeterminates. The following result shows that the differential Chow form can be obtained by intersecting $\mathcal{I}$ with $d+1$ generic $\delta$-hyperplanes.

Lemma 4.3. Using the notation introduced above, let $\mathcal{I}=\mathbb{I}(V)$ and

$$
\mathbb{P}_{i}=u_{i 0}+u_{i 1} y_{1}+\cdots+u_{i n} y_{n}(i=0, \ldots, d),
$$

where $u_{i j}$ are from (4.1). Then $\left[\mathcal{I}, \mathbb{P}_{0}, \mathbb{P}_{1}, \ldots, \mathbb{P}_{d}\right]$ is a prime $\delta$-ideal in $\mathcal{F}\langle\mathbf{u}\rangle\left\{u_{00}, u_{10}\right.$, $\left.\ldots, u_{d 0}, y_{1}, \ldots, y_{n}\right\}$ and $\left[\mathcal{I}, \mathbb{P}_{0}, \mathbb{P}_{1}, \ldots, \mathbb{P}_{d}\right] \cap \mathcal{F}\langle\mathbf{u}\rangle\left\{u_{00}, u_{10}, \ldots, u_{d 0}\right\}=\operatorname{sat}(f)$.

Proof. Similar to the proof of Lemma 3.5 , it is easy to show that $\left[\mathcal{I}, \mathbb{P}_{0}, \mathbb{P}_{1}, \ldots, \mathbb{P}_{d}\right]$ is a prime $\delta$-ideal with a generic zero $(\zeta, \xi)$. Denote $\left[\mathcal{I}, \mathbb{P}_{0}, \mathbb{P}_{1}, \ldots, \mathbb{P}_{d}\right]$ by $\mathbb{I}_{\zeta, \xi}$. Then, $\mathbb{I}_{\zeta, \xi} \cap \mathcal{F}\langle\mathbf{u}\rangle\left\{u_{00}, u_{10}, \ldots, u_{d 0}\right\}$ is a prime $\delta$-ideal with a generic zero $\zeta$, which implies $\mathbb{I}_{\zeta, \xi} \cap \mathcal{F}\langle\mathbf{u}\rangle\left\{u_{00}, u_{10}, \ldots, u_{d 0}\right\}=\mathbb{I}_{\zeta}=\operatorname{sat}(f)$.

In the following context, we will denote $\left[\mathcal{I}, \mathbb{P}_{0}, \mathbb{P}_{1}, \ldots, \mathbb{P}_{d}\right]$ by $\mathbb{I}_{\zeta, \xi}$.

Remark 4.4. From Lemma 4.3, we have two observations. First, the Chow form for a $\delta$-variety is independent of the generic point used in (4.2). The $\delta$-ideals $\mathbb{I}_{\zeta}$ and $\mathbb{I}_{\zeta, \xi}$ are also independent of the choice of $\xi$. Second, we can compute the Chow form of $V$ with the $\delta$-elimination algorithms [1, 7, 32, 39, 14, if we know a set of finitely many generating $\delta$-polynomials for $V$. Furthermore, given a characteristic set $\mathcal{A}$ of $\mathbb{I}(V)$, we can also compute its Chow form. Indeed, from Lemma 4.3 it suffices to compute a characteristic set of $\mathbb{I}_{\zeta, \xi}$ w.r.t. a ranking $\mathbb{U} \ll \mathbb{Y}$ (elimination ranking between elements of $\mathbb{U}=\left\{u_{00}, \ldots, u_{d 0}\right\}$ and $\mathbb{Y}$. It is clear that $\mathbb{I}_{\zeta, \xi}$ has a characteristic set $\left\{\mathcal{A}, \mathbb{P}_{0}, \ldots, \mathbb{P}_{d}\right\}$ w.r.t. a ranking $\mathbb{Y} \ll \mathbb{U}$. Then using the algorithms given by Boulier et al. [1] and Golubitsky et al. 14 for transforming a characteristic decomposition of a radical $\delta$-ideal from one ranking to another, we can obtain the Chow form.

Example 4.5. Consider the case $n=1$. By Theorem 2.9, a prime $\delta$-ideal in $\mathcal{F}\left\{y_{1}\right\}$ is of the form sat $(p)$ where $p \in \mathcal{F}\left\{y_{1}\right\}$ is irreducible. The zero set of $\operatorname{sat}(p)$ is an irreducible $\delta$ - $\mathcal{F}$-variety in the affine line: $\mathbb{A}^{1}(\mathcal{E})$. Let $\mathbb{P}_{0}=u_{00}+u_{01} y_{1}$ and $\mathbf{u}_{0}=\left(u_{00}, u_{01}\right)$. Then the Chow form of $\operatorname{sat}(p)$ is $F\left(\mathbf{u}_{0}\right)=u_{00}^{d} p\left(-\frac{u_{01}}{u_{00}}\right)$, where $d$ is a natural number such that $F\left(\mathbf{u}_{0}\right)$ is an irreducible $\delta$-polynomial in $\mathcal{F}\left\{\mathbf{u}_{0}\right\}$. For instance, let $p=\left(y_{1}^{\prime}\right)^{2}-4 y_{1}$. Then the Chow form of $\operatorname{sat}(p)$ is $F\left(\mathbf{u}_{0}\right)=$ $u_{01}^{2}\left(u_{00}^{\prime}\right)^{2}-2 u_{01} u_{01}^{\prime} u_{00} u_{00}^{\prime}+\left(u_{01}^{\prime}\right)^{2} u_{00}^{2}+4 u_{01}^{3} u_{00}$. 
Example 4.6. If $V$ is an irreducible $\delta$-variety of dimension $n-1$ and its corresponding prime $\delta$-ideal is $\mathcal{I}=\operatorname{sat}(p) \subset \mathcal{F}\{\mathbb{Y}\}$, then its Chow form is $F\left(\mathbf{u}_{0}, \ldots, \mathbf{u}_{n-1}\right)=$ $D^{m} p\left(\frac{D_{1}}{D}, \ldots, \frac{D_{n}}{D}\right)$, where

$$
D=\left|\begin{array}{cccc}
u_{01} & u_{02} & \cdots & u_{0 n} \\
u_{11} & u_{12} & \cdots & u_{1 n} \\
\ldots \ldots \ldots \ldots \ldots \ldots \ldots \ldots & \ldots \ldots \ldots \\
u_{n-1,1} & u_{n-1,2} & \cdots & u_{n-1, n}
\end{array}\right|
$$

and $D_{i}(i=1, \ldots, n)$ is the determinant of the matrix formed by replacing the $i$ th column of $D$ by the column vector $\left(-u_{00},-u_{10}, \ldots,-u_{n-1,0}\right)^{T}$, and $m$ is the minimal integer such that $D^{m} p\left(\frac{D_{1}}{D}, \ldots, \frac{D_{n}}{D}\right) \in \mathcal{F}\left\{\mathbf{u} ; u_{00}, \ldots, u_{d 0}\right\}$.

Example 4.7. Let $V$ be the irreducible $\delta$-variety corresponding to $\left[y_{1}^{\prime}+1, y_{2}^{\prime}\right] \in$ $\mathbb{Q}(t)\left\{y_{1}, y_{2}\right\}$. It is of dimension zero and the Chow form of $V$ is $F\left(\mathbf{u}_{0}\right)=u_{02} u_{01}^{\prime} u_{00}^{\prime \prime}+$ $u_{01}^{\prime \prime} u_{01} u_{02}-2 u_{02}\left(u_{01}^{\prime}\right)^{2}-u_{02} u_{01}^{\prime \prime} u_{00}^{\prime}-u_{02}^{\prime} u_{01} u_{00}^{\prime \prime}-u_{02}^{\prime \prime} u_{01}^{2}-u_{01}^{\prime} u_{02}^{\prime \prime} u_{00}+2 u_{02}^{\prime} u_{01} u_{01}^{\prime}+$ $u_{02}^{\prime \prime} u_{01} u_{00}^{\prime}+u_{01}^{\prime \prime} u_{02}^{\prime} u_{00}$, where $\mathbf{u}_{0}=\left(u_{00}, u_{01}, u_{02}\right)$.

4.2. The order of the differential Chow form. In this section, we will show that the order of the differential Chow form is the same as that of the corresponding $\delta$-variety. Before this, we give the following lemmas.

Lemma 4.8. Let $\zeta_{0}, \zeta_{1}, \ldots, \zeta_{d}$ be defined in (4.2), and $f\left(\mathbf{u} ; u_{00}, \ldots, u_{d 0}\right)$ be the Chow form of $V$. Then for any $p\left(\mathbf{u} ; u_{00}, \ldots, u_{d 0}\right) \in \mathcal{F}\langle\mathbf{u}\rangle\left\{u_{00}, \ldots, u_{d 0}\right\}$ with $\operatorname{ord}(f)$ $=\operatorname{ord}(p)$ such that $p\left(\mathbf{u} ; \zeta_{0}, \ldots, \zeta_{d}\right)=0$, we have $p\left(\mathbf{u} ; u_{00}, \ldots, u_{d 0}\right)=f\left(\mathbf{u} ; u_{00}, \ldots, u_{d 0}\right)$ $h\left(\mathbf{u} ; u_{00}, \ldots, u_{d 0}\right)$, where $h\left(\mathbf{u} ; u_{00}, \ldots, u_{d 0}\right)$ is in $\mathcal{F}\langle\mathbf{u}\rangle\left\{u_{00}, \ldots, u_{d 0}\right\}$.

Proof. Since $\{f\}$ is the characteristic set of $\mathbb{I}_{\zeta}$ w.r.t. an orderly ranking, and $p \in \mathbb{I}_{\zeta}$ with $\operatorname{ord}(f)=\operatorname{ord}(p)$, then $\mathrm{I}_{f}^{m} p=f h$ for some $m \in \mathbb{N}$. Since $f$ is irreducible, we can see that $f$ divides $p$.

The Chow form $f\left(\mathbf{u} ; u_{00}, \ldots, u_{d 0}\right)$ has certain symmetric properties as shown by the following results.

Lemma 4.9. Let $F\left(\mathbf{u}_{0}, \mathbf{u}_{1}, \ldots, \mathbf{u}_{d}\right)$ be the Chow form of an irreducible $\delta$ - $\mathcal{F}$-variety $V$ and $F^{*}\left(\mathbf{u}_{0}, \mathbf{u}_{1}, \ldots, \mathbf{u}_{d}\right)$ be obtained from $F$ by interchanging $\mathbf{u}_{\rho}$ and $\mathbf{u}_{\tau}$. Then $F^{*}$ and $F$ differ at most by a sign. Furthermore, $\operatorname{ord}\left(F, u_{i j}\right)(i=0, \ldots, d ; j=$ $0,1, \ldots, n)$ are the same for all $u_{i j}$ occurring in $F$. In particular, $u_{i 0}(i=0, \ldots, d)$ appear effectively in $F$. A necessary and sufficient condition for some $u_{i j}(j>0)$ not occurring effectively in $F$ is $y_{j} \in \mathbb{I}(V)$.

Proof. Consider the $\delta$-automorphism $\phi$ of $\mathcal{F}\left\langle\xi_{1}, \ldots, \xi_{n}\right\rangle\langle\mathbf{u}\rangle$ over $\mathcal{F}\left\langle\xi_{1}, \ldots, \xi_{n}\right\rangle$ :

$$
\phi\left(u_{i j}\right)=u_{i j}^{*}= \begin{cases}u_{i j}, & i \neq \rho, \tau, \\ u_{\tau j}, & i=\rho, \\ u_{\rho j}, & i=\tau .\end{cases}
$$

Of course,

$$
\phi\left(\zeta_{i}\right)=\zeta_{i}^{*}= \begin{cases}\zeta_{i}, & i \neq \rho, \tau, \\ \zeta_{\tau}, & i=\rho, \\ \zeta_{\rho}, & i=\tau .\end{cases}
$$

Then $\phi\left(f\left(\mathbf{u} ; \zeta_{0}, \ldots, \zeta_{\rho}, \ldots, \zeta_{\tau}, \ldots, \zeta_{d}\right)\right)=f\left(\mathbf{u}^{*} ; \zeta_{0}, \ldots, \zeta_{\tau}, \ldots, \zeta_{\rho}, \ldots, \zeta_{d}\right)=0$. Instead of $f\left(\mathbf{u} ; z_{0}, \ldots, z_{d}\right)$, we obtain another $\delta$-polynomial $p\left(\mathbf{u} ; z_{0}, \ldots, z_{d}\right)=f\left(\mathbf{u}^{*} ; z_{0}\right.$, $\left.\ldots, z_{\tau}, \ldots, z_{\rho}, \ldots, z_{d}\right) \in \mathbb{I}_{\zeta}$. Since the two $\delta$-polynomials $f$ and $p$ have the same order and degree, and as algebraic polynomials they have the same content, by Lemma 
4.8. $f\left(\mathbf{u}^{*} ; z_{0}, \ldots, z_{\tau}, \ldots, z_{\rho}, \ldots, z_{d}\right)$ can only differ by a sign with $f\left(\mathbf{u} ; z_{0}, \ldots, z_{\rho}, \ldots\right.$, $\left.z_{\tau}, \ldots, z_{d}\right)$. So we conclude that $F\left(\mathbf{u}_{0}, \mathbf{u}_{1}, \ldots, \mathbf{u}_{d}\right)$ produces at most a change of sign if we interchange $\mathbf{u}_{\rho}$ with $\mathbf{u}_{\tau}$. In particular, each $u_{i 0}$ appears effectively in $F$, and $\operatorname{ord}\left(F, u_{i 0}\right)$ are the same for all $i=0,1, \ldots, d$. Suppose $\operatorname{ord}\left(F, u_{i 0}\right)=s$. For $j \neq 0$, we consider $\operatorname{ord}\left(F, u_{i j}\right)$. If $\operatorname{ord}\left(F, u_{i j}\right)=l>s$, then we differentiate $f\left(\mathbf{u} ; \zeta_{0}, \ldots, \zeta_{d}\right)=0$ on both sides w.r.t. $u_{i j}^{(l)}$. Thus $\frac{\partial f}{\partial u_{i j}^{(l)}}\left(\mathbf{u} ; \zeta_{0}, \ldots, \zeta_{d}\right)=0$, which amounts to a contradiction by Lemma4.8. If $\operatorname{ord}\left(F, u_{i j}\right)=l<s$, then we differentiate $f\left(\mathbf{u} ; \zeta_{0}, \ldots, \zeta_{d}\right)=0$ on both sides w.r.t. $u_{i j}^{(s)}$. Thus $\frac{\partial f}{\partial u_{i 0}^{(s)}}\left(\mathbf{u} ; \zeta_{0}, \ldots, \zeta_{d}\right) \cdot\left(-\xi_{j}\right)=$ 0. Since $\frac{\partial f}{\partial u_{i 0}^{(s)}}\left(\mathbf{u} ; \zeta_{0}, \ldots, \zeta_{d}\right) \neq 0$, we have $\xi_{j}=0$. Also, $y_{j} \in \mathbb{I}(V) \Longleftrightarrow \xi_{j}=0 \Longleftrightarrow \zeta_{i}$ is free of $u_{i j} \Longleftrightarrow \frac{\partial f}{\partial u_{i j}^{(k)}}=0$ for all $k \in \mathbb{Z}^{+} \Longleftrightarrow u_{i j}$ does not appear in $F$. From the above, if $u_{i j}$ occurs effectively in $F$, ord $\left(F, u_{i j}\right)=s$, which completes the theorem.

The order of the Chow form is defined to be $\operatorname{ord}(f)=\operatorname{ord}(F)=\operatorname{ord}\left(f, u_{i 0}\right)$ for any $i \in\{0, \ldots, d\}$. By Lemma 4.9, $\operatorname{ord}(f)$ is equal to $\operatorname{ord}\left(F, u_{i j}\right)$ for those $u_{i j}$ occurring in $F$.

The following lemma gives a property for the $\delta$-ideal $\mathbb{I}_{\zeta, \xi}$ defined in Lemma 4.3.

Lemma 4.10. Let $F\left(\mathbf{u}_{0}, \mathbf{u}_{1}, \ldots, \mathbf{u}_{d}\right)=f\left(\mathbf{u} ; u_{00}, u_{10}, \ldots, u_{d 0}\right)$ be the Chow form of a prime $\delta$ - $\mathcal{F}$-ideal $\mathcal{I}$ and $s=\operatorname{ord}(f)$. Then

$$
\mathcal{A}=\left\{f, S_{f} y_{1}-\frac{\partial f}{\partial u_{01}^{(s)}}, \ldots, S_{f} y_{n}-\frac{\partial f}{\partial u_{0 n}^{(s)}}\right\}
$$

is a characteristic set of the prime $\delta$-ideal $\mathbb{I}_{\zeta, \xi}=\left[\mathcal{I}, \mathbb{P}_{0}, \mathbb{P}_{1}, \ldots, \mathbb{P}_{d}\right]$ in $\mathcal{F}\langle\mathbf{u}\rangle\left\{u_{00}, u_{10}\right.$, $\left.\ldots, u_{d 0}, \mathbb{Y}\right\}$ w.r.t. the elimination ranking $u_{d 0} \prec \cdots \prec u_{00} \prec y_{1} \prec \cdots \prec y_{n}$, where $S_{f}=\frac{\partial f}{\partial u_{00}^{(s)}}$.

Proof. From Lemma 4.3, $\mathbb{I}_{\zeta, \xi}$ is a prime $\delta$-ideal of dimension $d$ with a generic point $\left(\zeta_{0}, \ldots, \zeta_{d}, \xi_{1}, \ldots, \xi_{n}\right)$. From Lemma 4.1, $u_{10}, \ldots, u_{d 0}$ is a parametric set of $\mathbb{I}_{\zeta, \xi}$. If we differentiate $f\left(\mathbf{u} ; \zeta_{0}, \ldots, \zeta_{d}\right)=0$ w.r.t. $u_{0 \rho}^{(s)}(\rho=1, \cdots, n)$, then we have $\frac{\overline{\partial f}}{\partial u_{0 \rho}^{(s)}}-\xi_{\rho} \bar{S}_{f}=0$, where $\frac{\overline{\partial f}}{\partial u_{0 \rho}^{(s)}}$ and $\bar{S}_{f}$ are obtained by replacing $\left(u_{00}, \ldots, u_{d 0}\right)$ with $\left(\zeta_{0}, \ldots, \zeta_{d}\right)$ in $\frac{\partial f}{\partial u_{0 \rho}^{(s)}}$ and $S_{f}$, respectively. So $T_{\rho}=S_{f} y_{\rho}-\frac{\partial f}{\partial u_{0 \rho}^{(s)}} \in \mathbb{I}_{\zeta, \xi}$. Since $f$ is irreducible, we have $S_{f} \notin \mathbb{I}_{\zeta, \xi}$. Also note that $T_{i}$ is linear in $y_{i}$. $\mathcal{A}$ must be a characteristic set2 of $\mathbb{I}_{\zeta, \xi}$ w.r.t. the elimination ranking $u_{d 0} \prec \cdots \prec u_{00} \prec y_{1} \prec$ $\cdots \prec y_{n}$.

Now we give the first main property of the differential Chow form.

Theorem 4.11. Let $\mathcal{I}$ be a prime $\delta$ - $\mathcal{F}$-ideal with dimension $d$, and $f\left(\mathbf{u} ; u_{00}, u_{10}, \ldots\right.$, $\left.u_{d 0}\right)$ its Chow form. Then $\operatorname{ord}(f)=\operatorname{ord}(\mathcal{I})$.

Proof. Use the notation $\xi_{i}, \zeta_{i}$, and $\mathbb{P}_{i}$ introduced in (4.2) and (4.5). Let $\mathcal{I}_{d}=$ $\left[\mathcal{I}, \mathbb{P}_{1}, \ldots, \mathbb{P}_{d}\right] \subset \mathcal{F}\left\langle\mathbf{u}_{1}, \ldots, \mathbf{u}_{d}\right\rangle\{\mathbb{Y}\}$. By Theorem 3.14, $\mathcal{I}_{d}$ is a prime $\delta$-ideal with dimension 0 and the same order as $\mathcal{I}$.

Let $\mathcal{I}_{1}=\left[\mathcal{I}, \mathbb{P}_{0}, \ldots, \mathbb{P}_{d}\right]=\left[\mathcal{I}_{d}, \mathbb{P}_{0}\right] \subset \mathcal{F}\left\langle\mathbf{u}_{1}, \ldots, \mathbf{u}_{d} ; u_{01}, \ldots, u_{0 n}\right\rangle\left\{u_{00}, y_{1}, \ldots, y_{n}\right\}$. From Lemma 4.10, $\mathcal{A}=\left\{f, S_{f} y_{1}-\frac{\partial f}{\partial u_{01}^{(s)}}, \ldots, S_{f} y_{n}-\frac{\partial f}{\partial u_{0 n}^{(s)}}\right\}$ is a characteristic set

\footnotetext{
${ }^{2}$ Here $\mathcal{A}$ is a $\delta$-chain. See Remark 2.3
} 
of $\mathbb{I}_{\zeta, \xi}$. By Lemma 4.1, $u_{10}, \ldots, u_{d 0}$ is a parametric set of $\mathbb{I}_{\zeta, \xi}$. So $\mathcal{A}$ is also a characteristic set of $\mathcal{I}_{1}$ w.r.t. the elimination ranking $u_{00} \prec y_{1} \prec \cdots \prec y_{n}$. Since $\operatorname{dim}\left(\mathcal{I}_{1}\right)=0$, from Corollary 2.10, we have $\operatorname{ord}\left(\mathcal{I}_{1}\right)=\operatorname{ord}(\mathcal{A})=\operatorname{ord}(f)$.

On the other hand, if $\left(\eta_{1}, \ldots, \eta_{n}\right)$ is a generic point of $\mathcal{I}_{d}$, then $\left(\eta_{1}, \ldots, \eta_{n}, \zeta\right)$ is a generic point of $\mathcal{I}_{1}$ with $\zeta=-\sum_{j=1}^{n} u_{0 j} \eta_{j}$ and $\operatorname{dim}\left(\mathcal{I}_{1}\right)=0$. Clearly, $u_{0 k}(k=$ $1, \ldots, n)$ are $\delta$-independent over $\mathcal{F}\left\langle\mathbf{u}_{1}, \ldots, \mathbf{u}_{d}, \eta_{1}, \ldots, \eta_{n}\right\rangle$. Denote $\mathcal{F}\left\langle\mathbf{u}_{1}, \ldots, \mathbf{u}_{d}\right.$, $\left.u_{01}, \ldots, u_{0 n}\right\rangle$ by $\mathcal{F}_{1}$. So for a sufficiently large $t$,

$$
\begin{aligned}
& \omega_{\mathcal{I}_{1}}(t)=\operatorname{ord}\left(\mathcal{I}_{1}\right) \\
= & \operatorname{tr} \cdot \operatorname{deg} \mathcal{F}_{1}\left(\eta_{i}^{(j)}, \zeta^{(j)}: i=1, \ldots, n ; j \leq t\right) / \mathcal{F}_{1} \\
= & \operatorname{tr} \cdot \operatorname{deg} \mathcal{F}_{1}\left(\eta_{i}^{(j)}: i=1, \ldots, n ; j \leq t\right) / \mathcal{F}_{1} \\
= & \operatorname{tr} \cdot \operatorname{deg} \mathcal{F}\left\langle\mathbf{u}_{1}, \ldots, \mathbf{u}_{d}\right\rangle\left(\eta_{i}^{(j)}: i=1, \ldots, n ; j \leq t\right) / \mathcal{F}\left\langle\mathbf{u}_{1}, \ldots, \mathbf{u}_{d}\right\rangle \\
= & \omega_{\mathcal{I}_{d}}(t)=\operatorname{ord}\left(\mathcal{I}_{d}\right) .
\end{aligned}
$$

Thus, $\operatorname{ord}\left(\mathcal{I}_{1}\right)=\operatorname{ord}\left(\mathcal{I}_{d}\right)=\operatorname{ord}(\mathcal{I})$, and consequently, $\operatorname{ord}(\mathcal{I})=\operatorname{ord}(f)$.

As a consequence, we can give an equivalent definition for the order of a prime $\delta$-ideal using Chow forms.

Definition 4.12. Let $\mathcal{I}$ be a prime $\delta$-ideal in $\mathcal{F}\{\mathbb{Y}\}$ with dimension $d$ and $F\left(\mathbf{u}_{0}, \mathbf{u}_{1}\right.$, $\ldots, \mathbf{u}_{d}$ ) its Chow form. The order of $\mathcal{I}$ is defined to be the order of its Chow form.

The following result shows that we can recover the generic point $\left(\xi_{1}, \ldots, \xi_{n}\right)$ of $V$ from its Chow form.

Theorem 4.13. Let $f\left(\mathbf{u} ; u_{00}, \ldots, u_{d 0}\right)$ be defined as above and $h$ the order of $V$. Then we have

$$
\xi_{\rho}=\overline{\frac{\partial f}{\partial u_{0 \rho}^{(h)}}} / \bar{S}_{f}, \quad \rho=1, \ldots, n,
$$

where $\overline{\frac{\partial f}{\partial u_{0 \rho}^{(h)}}}$ and $\bar{S}_{f}$ are obtained by replacing $\left(u_{00}, \ldots, u_{d 0}\right)$ by $\left(\zeta_{0}, \ldots, \zeta_{d}\right)$ in $\frac{\partial f}{\partial u_{0 \rho}^{(h)}}$ and $\frac{\partial f}{\partial u_{00}^{(h)}}$, respectively.

Proof. It follows directly from Lemma 4.10 and Theorem 4.11 .

Remark 4.14. The $\delta$-resolvent [10] and [32, p.34] is closely related with the differential Chow form, although they are different. From Lemma 4.10 and Theorem 4.11 , we can see that both of them can be used to reduce a $\delta$-ideal $\mathcal{I}$ to a $\delta$-hypersurface which has the same order as $\mathcal{I}$ in a certain sense. But they are quite different. First, the resolvent depends on a parameter set $U$ of $\mathcal{I}$. To be more precise, let $Z=\mathbb{Y} \backslash U$. Then, the resolvent is essentially constructed in $\mathcal{F}\langle U\rangle\{Z\}$. Second, to define the resolvent, we need only one new indeterminate $w$ and add one linear $\delta$-polynomial $w-c_{1} v_{1}-\cdots-c_{p} v_{p}$ to $\mathcal{I}$, where $Z=\left\{v_{1}, \ldots, v_{p}\right\}$. Furthermore, $c_{i}$ in the above expression are from $\mathcal{F}$. Therefore, the resolvent will never be the Chow form. Of course, if $\mathcal{I}$ is of dimension zero and we take $c_{i}$ in $w-c_{1} y_{1}-\cdots-c_{n} y_{n}$ as $\delta$-inderterminates, then the resolvent is the Chow form of $\mathcal{I}$. On the other hand, the resolvent of $\mathcal{I}$ can be obtained from its Chow form by specializing some $u_{i j}$ to certain specific values and using techniques in Theorem 2.16 . 
4.3. Differential Chow form is differentially homogenous. Following Kolchin [23], we introduce the concept of $\delta$-homogenous $\delta$-polynomials.

Definition 4.15. A $\delta$-polynomial $p \in \mathcal{F}\left\{y_{0}, y_{1}, \ldots, y_{n}\right\}$ is called $\delta$-homogenous of degree $m$ if for a new $\delta$ - $\mathcal{F}$-indeterminate $\lambda$, we have $p\left(\lambda y_{0}, \lambda y_{1}, \ldots, \lambda y_{n}\right)=$ $\lambda^{m} p\left(y_{0}, y_{1}, \ldots, y_{n}\right)$.

The differential analog of Euler's theorem related to homogenous polynomials is valid.

Theorem 4.16 ([19, p.71]). A $\delta$-polynomial $f \in \mathcal{F}\left\{y_{0}, y_{1}, \ldots, y_{n}\right\}$ is $\delta$-homogenous of degree $m$ if and only if

$$
\sum_{j=0}^{n} \sum_{k \in \mathbb{N}}\left(\begin{array}{c}
k+r \\
r
\end{array}\right) y_{j}^{(k)} \frac{\partial f\left(y_{0}, \ldots, y_{n}\right)}{\partial y_{j}^{(k+r)}}=\left\{\begin{array}{cc}
m f, & r=0 \\
0, & r \neq 0
\end{array}\right.
$$

For the Chow form, we have the following result.

Theorem 4.17. Let $F\left(\mathbf{u}_{0}, \mathbf{u}_{1}, \ldots, \mathbf{u}_{d}\right)=f\left(\mathbf{u} ; u_{00}, \ldots, u_{d 0}\right)$ be the Chow form of an irreducible $\delta$-variety $V$ of dimension d and order $h$. Then

1)

$$
\sum_{j=0}^{n} u_{\tau j} \frac{\partial f}{\partial u_{\sigma j}}+\sum_{j=0}^{n} u_{\tau j}^{\prime} \frac{\partial f}{\partial u_{\sigma j}^{\prime}}+\cdots+\sum_{j=0}^{n} u_{\tau j}^{(h)} \frac{\partial f}{\partial u_{\sigma j}^{(h)}}= \begin{cases}0, & \sigma \neq \tau \\ r f, & \sigma=\tau\end{cases}
$$

where $r$ is a nonnegative integer.

2) $F\left(\mathbf{u}_{0}, \mathbf{u}_{1}, \ldots, \mathbf{u}_{d}\right)$ is a $\delta$-homogenous $\delta$-polynomial of degree $r$ in each set $\mathbf{u}_{i}$ and $F$ is of total degree $(d+1) r$.

Proof. Let $\left(\xi_{1}, \ldots, \xi_{n}\right)$ be a generic point of $V$ and $\zeta_{i}=-\sum_{j=1}^{n} u_{i j} \xi_{j}(i=0, \ldots, d)$ defined in (4.2). From (4.3), $f\left(\mathbf{u} ; u_{00}, \ldots, u_{d 0}\right)$ is the characteristic set of the prime $\delta$-ideal $\mathbb{I}_{\zeta}$. Since $f\left(\mathbf{u} ; \zeta_{0}, \zeta_{1}, \ldots, \zeta_{d}\right)=0$, we have

$$
\begin{aligned}
& \overline{\frac{\partial f}{\partial u_{\sigma j}}}+\frac{\partial f}{\partial \zeta_{\sigma}}\left(-\xi_{j}\right)+\frac{\partial f}{\partial \zeta_{\sigma}^{\prime}}\left(-\xi_{j}^{\prime}\right)+\frac{\partial f}{\partial \zeta_{\sigma}^{\prime \prime}}\left(-\left(\begin{array}{l}
2 \\
0
\end{array}\right) \xi_{j}^{\prime \prime}\right)+\ldots+\frac{\partial f}{\partial \zeta_{\sigma}^{(h)}}\left[-\left(\begin{array}{l}
h \\
0
\end{array}\right) \xi_{j}^{(h)}\right]=0 \\
& \frac{\partial f}{\partial u_{\sigma j}^{\prime}}+\quad 0+\frac{\partial f}{\partial \zeta_{\sigma}^{\prime}}\left(-\xi_{j}\right)+\frac{\partial f}{\partial \zeta_{\sigma}^{\prime \prime}}\left(-\left(\begin{array}{l}
2 \\
1
\end{array}\right) \xi_{j}^{\prime}\right)+\ldots+\frac{\partial f}{\partial \zeta_{\sigma}^{(h)}}\left[-\left(\begin{array}{c}
h \\
1
\end{array}\right) \xi_{j}^{(h-1)}\right]=0 \\
& \frac{\partial f}{\partial u_{\sigma j}^{\prime \prime}}+0+0+\frac{\partial f}{\partial \zeta_{\sigma}^{\prime \prime}}\left(-\left(\begin{array}{c}
2 \\
2
\end{array}\right) \xi_{j}\right)+\ldots+\frac{\partial f}{\partial \zeta_{\sigma}^{(h)}}\left[-\left(\begin{array}{c}
h \\
2
\end{array}\right) \xi_{j}^{(h-2)}\right]=0
\end{aligned}
$$

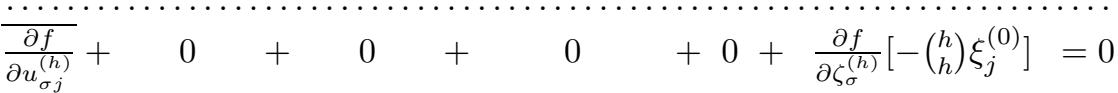

In the above equations, $\frac{\overline{\partial f}}{\partial u_{\sigma j}^{(l)}}$ and $\frac{\partial f}{\partial \zeta_{\sigma}^{(l)}}(l=0, \ldots, h ; j=1, \ldots, n)$ are obtained by substituting $\zeta_{i}$ to $u_{i 0}(i=0,1, \ldots, d)$ in each $\frac{\partial f}{\partial u_{\sigma j}^{(l)}}$ and $\frac{\partial f}{\partial u_{\sigma 0}^{(l)}}$, respectively.

Now, let us consider the $\delta$-polynomial $\sum_{j=0}^{n} \sum_{k \geq 0}\left(\begin{array}{c}k+i \\ k\end{array}\right) u_{\sigma j}^{(k)} \frac{\partial f}{\partial u_{\sigma j}^{(k+i)}}$.

In the case $i=0$, first, let $(0 *) \times u_{\tau j}+(1 *) \times u_{\tau j}^{\prime}+\cdots+(h *) \times u_{\tau j}^{(h)}$ and add them together for $j$ from 1 to $n$. We obtain

$\sum_{j=1}^{n} u_{\tau j} \frac{\overline{\partial f}}{\partial u_{\sigma j}}+\sum_{j=1}^{n} u_{\tau j}^{\prime} \frac{\overline{\partial f}}{\partial u_{\sigma j}^{\prime}}+\cdots+\sum_{j=1}^{n} u_{\tau j}^{(h)} \frac{\overline{\partial f}}{\partial u_{\sigma j}^{(h)}}+\zeta_{\tau} \frac{\partial f}{\partial \zeta_{\sigma}}+\zeta_{\tau}^{\prime} \frac{\partial f}{\partial \zeta_{\sigma}^{\prime}}+\cdots+\zeta_{\tau}^{(h)} \frac{\partial f}{\partial \zeta_{\sigma}^{(h)}}=0$.

So the $\delta$-polynomial $\sum_{j=0}^{n} u_{\tau j} \frac{\partial f}{\partial u_{\sigma j}}+\sum_{j=0}^{n} u_{\tau j}^{\prime} \frac{\partial f}{\partial u_{\sigma j}^{\prime}}+\sum_{j=0}^{n} u_{\tau j}^{\prime \prime} \frac{\partial f}{\partial u_{\sigma j}^{\prime \prime}}+\cdots+\sum_{j=0}^{n} u_{\tau j}^{(h)} \frac{\partial f}{\partial u_{\sigma j}^{(h)}}$ vanishes at $\left(u_{00}, \ldots, u_{d 0}\right)=\left(\zeta_{0}, \ldots, \zeta_{d}\right)$. Thus in the case $\tau=\sigma$, it can be divisible 
by $f$, i.e. $\sum_{j=0}^{n} \sum_{k=0}^{h} u_{\sigma j}^{(k)} \frac{\partial f}{\partial u_{\sigma j}^{(k)}}=r f$. By Euler's theorem, $f$ is an algebraic homogenous $\delta$-polynomial of degree $r$ in each set of indeterminates $\mathbf{u}_{i}=\left(u_{i 0}, \ldots, u_{i n}\right)$ and their derivatives. But in the case $\tau \neq \sigma$, since this $\delta$-polynomial is of order not greater than $f$ and cannot be divisible by $f$, by Lemma 4.8, it must be identically zero. Thus, we have proved 1) of the Theorem.

In the case $i \neq 0$,

$$
\begin{aligned}
0= & (i *) \times\left(\begin{array}{l}
i \\
i
\end{array}\right) u_{\sigma j}+(i+1 *) \times\left(\begin{array}{c}
i+1 \\
i
\end{array}\right) u_{\sigma j}^{\prime}+\cdots+(h *) \times\left(\begin{array}{c}
h \\
i
\end{array}\right) u_{\sigma j}^{(h-i)} \\
= & \left(\begin{array}{c}
i \\
i
\end{array}\right) u_{\sigma j} \frac{\partial f}{\partial u_{\sigma j}^{(i)}}+\left(\begin{array}{c}
i+1 \\
i
\end{array}\right) u_{\sigma j}^{\prime} \frac{\partial f}{\partial u_{\sigma j}^{(i+1)}}+\cdots+\left(\begin{array}{c}
h \\
i
\end{array}\right) u_{\sigma j}^{(h-i)} \frac{\overline{\partial f}}{\partial u_{\sigma j}^{(h)}} \\
& +\frac{\partial f}{\partial \zeta_{\sigma}^{(i)}}\left(-\left(\begin{array}{c}
i \\
i
\end{array}\right) u_{\sigma j} \xi_{j}\right)+\frac{\partial f}{\partial \zeta_{\sigma}^{(i+1)}}\left(-\left(\begin{array}{c}
i+1 \\
i
\end{array}\right) u_{\sigma j} \xi_{j}^{\prime}-\left(\begin{array}{c}
i+1 \\
i
\end{array}\right)\left(\begin{array}{c}
i+1 \\
i+1
\end{array}\right) u_{\sigma j}^{\prime} \xi_{j}\right) \\
& +\cdots \cdots \\
& +\frac{\partial f}{\partial \zeta_{\sigma}^{(h)}}\left(-\left(\begin{array}{c}
i \\
i
\end{array}\right)\left(\begin{array}{c}
h \\
i
\end{array}\right) u_{\sigma j} \xi_{j}^{(h-i)}-\left(\begin{array}{c}
i+1 \\
i
\end{array}\right)\left(\begin{array}{c}
h \\
i+1
\end{array}\right) u_{\sigma j}^{\prime} \xi_{j}^{(h-i-1)}-\cdots-\left(\begin{array}{c}
h \\
i
\end{array}\right)\left(\begin{array}{c}
h \\
h
\end{array}\right) u_{\sigma j}^{(h-i)} \xi_{j}\right) \\
= & \left(\begin{array}{c}
i \\
i
\end{array}\right) u_{\sigma j} \frac{\partial f f}{\partial u_{\sigma j}^{(i)}}+\left(\begin{array}{c}
i+1 \\
i
\end{array}\right) u_{\sigma j}^{\prime} \frac{\partial f}{\partial u_{\sigma j}^{(i+1)}}+\cdots+\left(\begin{array}{c}
h \\
i
\end{array}\right) u_{\sigma j}^{(h-i)} \frac{\partial f}{\partial u_{\sigma j}^{(h)}} \\
& +\left(\begin{array}{l}
i \\
i
\end{array}\right) \frac{\partial f}{\partial \zeta_{\sigma}^{(i)}}\left(-u_{\sigma j} \xi_{j}\right)+\left(\begin{array}{c}
i+1 \\
i
\end{array}\right) \frac{\partial f}{\partial \zeta_{\sigma}^{(i+1)}}\left(-u_{\sigma j} \xi_{j}\right)^{\prime}+\cdots+\left(\begin{array}{c}
h \\
i
\end{array}\right) \frac{\partial f}{\partial \zeta_{\sigma}^{(h)}}\left(-u_{\sigma j} \xi_{j}\right)^{(h-i)} .
\end{aligned}
$$

Therefore, $\sum_{j=1}^{n}\left(\begin{array}{l}i \\ i\end{array}\right) u_{\sigma j} \overline{\frac{\partial f}{\partial u_{\sigma j}^{(i)}}}+\sum_{j=1}^{n}\left(\begin{array}{c}i+1 \\ i\end{array}\right) u_{\sigma j}^{\prime} \overline{\frac{\partial f}{\partial u_{\sigma j}^{(i+1)}}}+\cdots+\sum_{j=1}^{n}\left(\begin{array}{c}h \\ i\end{array}\right) u_{\sigma j}^{(h-i)} \overline{\frac{\partial f}{\partial u_{\sigma j}^{(h)}}}+$ $\left(\begin{array}{l}i \\ i\end{array}\right) \zeta_{\sigma} \frac{\partial f}{\partial \zeta_{\sigma}^{(i)}}+\left(\begin{array}{c}i+1 \\ i\end{array}\right) \zeta_{\sigma}^{\prime} \frac{\partial f}{\partial \zeta_{\sigma}^{(i+1)}}+\cdots+\left(\begin{array}{c}h \\ i\end{array}\right) \zeta_{\sigma}^{(h-i)} \frac{\partial f}{\partial \zeta_{\sigma}^{(h)}}=0$.

Thus, the $\delta$-polynomial $\sum_{j=0}^{n}\left(\begin{array}{c}i \\ i\end{array}\right) u_{\sigma j} \frac{\partial f}{\partial u_{\sigma j}^{(i)}}+\sum_{j=0}^{n}\left(\begin{array}{c}i+1 \\ i\end{array}\right) u_{\sigma j}^{\prime} \frac{\partial f}{\partial u_{\sigma j}^{(i+1)}}+\cdots+\sum_{j=0}^{n}\left(\begin{array}{c}h \\ i\end{array}\right)$ $u_{\sigma j}^{(h-i)} \frac{\partial f}{\partial u_{\sigma j}^{(h)}}$ is identically zero, for it vanishes at $\left(u_{00}, \ldots, u_{d 0}\right)=\left(\zeta_{0}, \ldots, \zeta_{d}\right)$ and cannot be divisible by $f$.

From the above, we conclude that

$$
\sum_{j=0}^{n} \sum_{k \geq 0}\left(\begin{array}{c}
k+i \\
i
\end{array}\right) u_{\sigma j}^{(k)} \frac{\partial f}{\partial u_{\sigma j}^{(k+i)}}=\left\{\begin{array}{cc}
0, & i \neq 0, \\
r f, & i=0 .
\end{array}\right.
$$

From Theorem 4.16 and the symmetry property of $F\left(\mathbf{u}_{0}, \ldots, \mathbf{u}_{d}\right)$, the theorem is obtained.

Lemma 4.9, Theorem 4.11, and Theorem 4.17 together prove the first property of Theorem 1.2 .

Remark 4.18. Using the terminology from [24, the differential Chow form $F\left(\mathbf{u}_{0}, \mathbf{u}_{1}\right.$, $\left.\ldots, \mathbf{u}_{d}\right)$ is $(d+1)$ - $\delta$-homogenous in $\left(\mathbf{u}_{0}, \mathbf{u}_{1}, \ldots, \mathbf{u}_{d}\right)$.

Definition 4.19. Let $\mathcal{I}$ be a prime $\delta$-ideal in $\mathcal{F}\{\mathbb{Y}\}$ of dimension $d$ and $F\left(\mathbf{u}_{0}, \mathbf{u}_{1}\right.$, $\left.\ldots, \mathbf{u}_{d}\right)$ its Chow form. The $\delta$-degree of $\mathcal{I}$ is defined to be the $\delta$-homogenous degree $r$ of its Chow form in each $\mathbf{u}_{i}(i=0, \ldots, d)$.

The following result shows that the $\delta$-degree of a $\delta$-variety $V$ is an invariant of $V$ under invertible linear transformations.

Lemma 4.20. Let $A=\left(a_{i j}\right)$ be an $n \times n$ invertible matrix with $a_{i j} \in \mathcal{F}$ and $F\left(\mathbf{u}_{0}, \mathbf{u}_{1}, \ldots, \mathbf{u}_{d}\right)$ be the Chow form of an irreducible $\delta$-variety $V$ with dimension $d$. Then the Chow form of the image $\delta$-variety of $V$ under the linear transformation 
$\mathbb{Y}=A \mathbb{X}$ is $F^{A}\left(\mathbf{v}_{0}, \ldots, \mathbf{v}_{d}\right)=F\left(\mathbf{v}_{0} B, \ldots, \mathbf{v}_{d} B\right)$, where $B=\left(\begin{array}{cc}1 & \\ 0 & 0 \cdots 0 \\ \vdots & \stackrel{A}{0}\end{array}\right)$ and $\mathbf{u}_{i}$ and $\mathbf{v}_{i}$ are regarded as row vectors.

Proof. Let $\xi=\left(\xi_{1}, \ldots, \xi_{n}\right)$ be a generic point of $V$. Under the linear transformation $\mathbb{Y}=A \mathbb{X}, \xi$ is mapped to $\eta=\left(\eta_{1}, \ldots, \eta_{n}\right)$ with $\eta_{i}=\sum_{j=1}^{n} a_{i j} \xi_{j}$. Under this transformation $V$ is mapped to a $\delta$-variety $V^{A}$ whose generic point is $\eta$. Note that $F^{A}\left(\mathbf{v}_{0}, \ldots, \mathbf{v}_{d}\right)=f^{A}\left(v_{i j} ; v_{00}, \ldots, v_{d 0}\right)=f\left(\sum_{k=1}^{n} v_{i k} a_{k j} ; v_{00}, \ldots, v_{d 0}\right)$ and $f^{A}\left(v_{i j} ;-\sum_{k=1}^{n} v_{0 k} \eta_{k}, \ldots,-\sum_{k=1}^{n} v_{d k} \eta_{k}\right)=f\left(\sum_{k=1}^{n} v_{i k} a_{k j} ;-\sum_{k=1}^{n} v_{0 k} \eta_{k}, \ldots\right.$, $\left.-\sum_{k=1}^{n} v_{d k} \eta_{k}\right)=f\left(\sum_{k=1}^{n} v_{i k} a_{k j} ;-\sum_{j=1}^{n}\left(\sum_{k=1}^{n} v_{0 k} a_{k j}\right) \xi_{j}, \ldots\right.$, $\left.-\sum_{j=1}^{n}\left(\sum_{k=1}^{n} v_{d k} a_{k j}\right) \xi_{j}\right)=0$. Since $V^{A}$ is of the same dimension and order as $V$ and $F^{A}$ is irreducible, from the definition of the Chow form, the claim is proved.

Definition 4.21. Let $p$ be a $\delta$-polynomial in $\mathcal{F}\{\mathbb{Y}\}$. Then the smallest number $r$ such that $y_{0}^{r} p\left(y_{1} / y_{0}, \ldots, y_{n} / y_{0}\right) \in \mathcal{F}\left\{y_{0}, y_{1}, \ldots, y_{n}\right\}$ is called the denomination of $p$, which is denoted by $\operatorname{den}(p)$.

Example 4.22. In the case $d=n-1$ and $n>1$, if $\left\{p\left(y_{1}, \ldots, y_{n}\right)\right\}$ is a characteristic set of $\mathcal{I}$ w.r.t. any ranking, then by Example 4.6 the $\delta$-degree of $\mathcal{I}$ cannot exceed the denomination of $p$. So the denomination of $p$ gives an upper bound of the $\delta$-degree of $\mathcal{I}$. But, we do not know whether they are the same.

Example 4.23. In the case $n=1$, we have $d=0$. If $\{p(y)\}$ is a characteristic set of $\mathcal{I}$ w.r.t. any ranking, then the $\delta$-degree of $\mathcal{I}$ is exactly equal to the denomination of $p$. Now we can give a bound for the $\delta$-degree of $\mathcal{I}$ from the original equation of $p$ without computing its denomination.

For a $\delta$-monomial $\theta(y)=y^{l_{0}}\left(y^{\prime}\right)^{l_{1}}\left(y^{\prime \prime}\right)^{l_{2}} \ldots\left(y^{(s)}\right)^{l_{s}}$, define its weighted degree to be $l_{0}+2 l_{1}+\cdots+(s+1) l_{s}$, denoted by wdeg $(\theta(y))=l_{0}+2 l_{1}+\cdots+(s+1) l_{s}$. For a $\delta$-polynomial $p \in \mathcal{F}\{y\}$, we can define its weighted degree to be the maximum of the weighted degrees of all the $\delta$-monomials effectively appearing in $p$. Clearly, the denomination of $p$ cannot exceed its weighted degree, and we have examples for which $\operatorname{den}(p)<\operatorname{wdeg}(p)$. Let $p=2 y^{\prime 2}-y y^{\prime \prime}$. Then, $\operatorname{den}(p)=3$ and $\operatorname{wdeg}(p)=4$. The Chow form of $\operatorname{sat}(p)$ is $F(\mathbf{u})=u_{0} u_{1} u_{0}^{\prime \prime}-u_{0}^{2} u_{1}^{\prime \prime}-2 u_{0}^{\prime 2} u_{1}+2 u_{0} u_{0}^{\prime} u_{1}^{\prime}$, where $\mathbf{u}=\left(u_{0}, u_{1}\right)$. So the $\delta$-degree of $\mathcal{I}=\operatorname{sat}(p)$ is 3 , which is less than wdeg $(p)$.

Now we contrast the above $p$ with a $\delta$-polynomial $q=y^{\prime 2}-y y^{\prime \prime}$ that is different from $p$ by only a single coefficient. Then $\operatorname{den}(q)=4$ and $\operatorname{wdeg}(q)=4$. The Chow form of $\operatorname{sat}(q)$ is $G\left(\mathbf{u}_{0}\right)=u_{0} u_{1}^{2} u_{0}^{\prime \prime}-u_{0}^{2} u_{1} u_{1}^{\prime \prime}-u_{0}^{\prime 2} u_{1}^{2}+u_{0}^{2} u_{1}^{\prime 2}$, so the $\delta$-degree of $\operatorname{sat}(q)$ is 4 , which is equal to the weighted degree of $q$. Thus, the weighted degree is a sharp bound.

4.4. Factorization of the differential Chow form. In the algebraic case, the Chow form can be factored into the product of linear polynomials with the generic points of the variety as coefficients. In this section, we will show that there is a differential analog to this result.

Let

$$
\tilde{\mathbf{u}}=\bigcup_{i=0}^{d} \mathbf{u}_{i} \backslash\left\{u_{00}\right\}
$$

and $\mathcal{F}_{0}=\mathcal{F}\langle\tilde{\mathbf{u}}\rangle\left(u_{00}^{(0)}, \ldots, u_{00}^{(h-1)}\right)$. Consider the Chow form $f\left(\mathbf{u} ; u_{00}, u_{10}, \ldots, u_{d 0}\right)$ as an irreducible algebraic polynomial $p\left(u_{00}^{(h)}\right)$ in $\mathcal{F}_{0}\left[u_{00}^{(h)}\right]$. Let $g=\operatorname{deg}\left(p, u_{00}^{(h)}\right)=$ 
$\operatorname{deg}\left(f, u_{00}^{(h)}\right)$. In a suitable algebraic extension field of $\mathcal{F}_{0}, p\left(u_{00}^{(h)}\right)=0$ has $g$ roots $\gamma_{1}, \ldots, \gamma_{g}$. Thus

$$
f\left(\mathbf{u} ; u_{00}, u_{10}, \ldots, u_{d 0}\right)=A\left(\mathbf{u}_{0}, \mathbf{u}_{1}, \ldots, \mathbf{u}_{d}\right) \prod_{\tau=1}^{g}\left(u_{00}^{(h)}-\gamma_{\tau}\right),
$$

where $A\left(\mathbf{u}_{0}, \mathbf{u}_{1}, \ldots, \mathbf{u}_{d}\right)$ is in $\mathcal{F}\left\{\mathbf{u}_{0}, \ldots, \mathbf{u}_{d}\right\}$. For each $\tau$ such that $1 \leq \tau \leq g$, let

$$
\mathcal{F}_{\tau}=\mathcal{F}_{0}\left(\gamma_{\tau}\right)=\mathcal{F}\langle\tilde{\mathbf{u}}\rangle\left(u_{00}^{(0)}, \ldots, u_{00}^{(h-1)}, \gamma_{\tau}\right)
$$

be an algebraic extension of $\mathcal{F}_{0}$ defined by $p\left(u_{00}^{(h)}\right)=0$. We will define a derivation $\delta_{\tau}$ on $\mathcal{F}_{\tau}$ so that $\left(\mathcal{F}_{\tau}, \delta_{\tau}\right)$ becomes a differential field. This can be done in a very natural way. For $e \in \mathcal{F}\langle\tilde{\mathbf{u}}\rangle$, define $\delta_{\tau} e=\delta e=e^{\prime}$. Define $\delta_{\tau}^{i} u_{00}=u_{00}^{(i)}$ for $i=0, \ldots, h-1$ and

$$
\delta_{\tau}^{h} u_{00}=\gamma_{\tau}
$$

Since $f$, regarded as an algebraic polynomial $p$ in $u_{00}^{(h)}$, is a minimal polynomial of $\gamma_{\tau}, \mathrm{S}_{f}=\frac{\partial f}{\partial u_{00}^{(h)}}$ does not vanish at $u_{00}^{(h)}=\gamma_{\tau}$. Now, we define the derivatives of $\delta_{\tau}^{i} u_{00}$ for $i>h$ by induction. First, since $p\left(\gamma_{\tau}\right)=0, \delta_{\tau}\left(p\left(\gamma_{\tau}\right)\right)=\left.\mathrm{S}_{f}\right|_{u_{00}^{(h)}=\gamma_{\tau}} \delta_{\tau}\left(\gamma_{\tau}\right)+$ $\left.T\right|_{u_{00}^{(h)}=\gamma_{\tau}}=0$, where $T=f^{\prime}-\mathrm{S}_{f} u_{00}^{(h+1)}$. We define $\delta_{\tau}^{h+1} u_{00}$ to be $\delta_{\tau}\left(\gamma_{\tau}\right)=$ $-\left.\frac{T}{\mathrm{~S}_{f}}\right|_{u_{00}^{(h)}=\gamma_{\tau}}$. Supposing the derivatives of $\delta_{\tau}^{j} u_{00}$ with order less than $j<i$ have been defined, we now define $\delta_{\tau}^{i} u_{00}$. Since $f^{(i)}=\mathrm{S}_{f} u_{00}^{(h+i)}+T_{i}$ is linear in $u_{00}^{(h+i)}$, we define $\delta_{\tau}^{i} u_{00}$ to be $-\left.\frac{T_{i}}{\mathrm{~S}_{f}}\right|_{u_{00}^{(h+j)}=\delta_{\tau}^{h+j} u_{00}, j<i}$.

In this way, $\left(\mathcal{F}_{\tau}, \delta_{\tau}\right)$ is a differential field which can be considered as a finitely differential extension field of $(\mathcal{F}\langle\tilde{\mathbf{u}}\rangle, \delta)$. Recall that $\mathcal{F}\langle\tilde{\mathbf{u}}\rangle$ is a finitely $\delta$-extension field of $\mathcal{F}$ contained in $\mathcal{E}$. By the definition of a universal $\delta$-extension field, there exists a $\delta$-extension field $\mathcal{F}^{*} \subset \mathcal{E}$ of $\mathcal{F}\langle\tilde{\mathbf{u}}\rangle$ and a $\delta$-isomorphism $\varphi_{\tau}$ over $\mathcal{F}\langle\tilde{\mathbf{u}}\rangle$ from $\left(\mathcal{F}_{\tau}, \delta_{\tau}\right)$ to $\left(\mathcal{F}^{*}, \delta\right)$. Summing up the above results, we have

Lemma 4.24. $\left(\mathcal{F}_{\tau}, \delta_{\tau}\right)$ defined above is a finitely differential extension field of $\mathcal{F}\langle\tilde{\mathbf{u}}\rangle$, which is $\delta$-F $\mathcal{F}\langle\tilde{\mathbf{u}}\rangle$-isomorphic to a subfield of $\mathcal{E}$.

Let $p$ be a $\delta$-polynomial in $\mathcal{F}\left\{\mathbf{u}_{0}, \mathbf{u}_{1}, \ldots, \mathbf{u}_{d}\right\}=\mathcal{F}\left\{\tilde{\mathbf{u}}, u_{00}\right\}$. For convenience, by the symbol $\left.p\right|_{u_{00}^{(h)}=\gamma_{\tau}}$ or, say, replacing $u_{00}^{(h)}$ by $\gamma_{\tau}$, we mean substituting $u_{00}^{(h+i)}$ by $\delta_{\tau}^{i} \gamma_{\tau}(i \geq 0)$ in $p$. Similarly, by saying $p$ vanishes at $u_{00}^{(h)}=\gamma_{\tau}$, we mean $\left.p\right|_{u_{00}^{(h)}=\gamma_{\tau}}=0$. It is easy to prove the following lemma.

Lemma 4.25. Let $p$ be a $\delta$-polynomial in $\mathcal{F}\left\{\tilde{\mathbf{u}}, u_{00}\right\}$ and $f$ the Chow form for a prime $\delta$-F-ideal $\mathcal{I}$ of dimension $d$. Then $p \in \operatorname{sat}(f)$ if and only if $p$ vanishes at $u_{00}^{(h)}=\gamma_{\tau}$.

When a $\delta$-polynomial $h \in \mathcal{F}\langle\tilde{\mathbf{u}}\rangle\{\mathbb{Y}\}$ vanishes at a point $\eta \in \mathcal{F}_{\tau}^{n}$, it is easy to see that $h$ vanishes at $\varphi_{\tau}(\eta) \in \mathcal{E}^{n}$. For convenience, by saying $\eta$ is in a $\delta$-variety $V$ over $\mathcal{F}\langle\tilde{\mathbf{u}}\rangle$, we mean $\varphi_{\tau}(\eta) \in V$.

Remark 4.26. In order to make $\mathcal{F}_{\tau}$ a differential field, we need to introduce a differential operator $\delta_{\tau}$ which is related to $\gamma_{\tau}$, and there does not exist a single differential operator to make all $\mathcal{F}_{\tau}(\tau=1, \ldots, g)$ differential fields. This natural 
phenomenon related with nonlinear differential equations seems not to have been used before. For instance, let $p=y^{\prime 2}-4 y$. Then $\mathcal{I}=\operatorname{sat}(p)$ is a prime $\delta$-ideal in $\mathbb{Q}(t)\{y\}$ and let $\overline{\mathcal{F}}$ be the differential rational function field of $\mathcal{I}$. By factoring $p=\left(y^{\prime}-2 \sqrt{y}\right)\left(y^{\prime}+2 \sqrt{y}\right)$, we can define two more differential fields: $\mathcal{F}_{1}=\mathbb{Q}(t)(\sqrt{y})$ with a differential operator $\delta_{1} y=2 \sqrt{y}$ and $\mathcal{F}_{2}=\mathbb{Q}(t)(\sqrt{y})$ with a differential operator $\delta_{2} y=-2 \sqrt{y}$. Note that $\mathcal{F}_{1}$ and $\mathcal{F}_{2}$ are not compatible, although each of them is isomorphic to a subfield of $\mathcal{E}$. Finally, both $\mathcal{F}_{1}$ and $\mathcal{F}_{2}$ are isomorphic to $\overline{\mathcal{F}}$.

With these preparations, we now give the following theorem.

Theorem 4.27. Let $F\left(\mathbf{u}_{0}, \mathbf{u}_{1}, \ldots, \mathbf{u}_{d}\right)=f\left(\mathbf{u} ; u_{00}, \ldots, u_{d 0}\right)$ be the Chow form of an irreducible $\delta-\mathcal{F}$-variety of dimension $d$ and order $h$. Then, there exist $\xi_{\tau 1}, \ldots, \xi_{\tau n}$ in a differential extension field $\left(\mathcal{F}_{\tau}, \delta_{\tau}\right)(\tau=1, \ldots, g)$ of $(\mathcal{F}\langle\tilde{\mathbf{u}}\rangle, \delta)$ such that

$$
F\left(\mathbf{u}_{0}, \mathbf{u}_{1}, \ldots, \mathbf{u}_{d}\right)=A\left(\mathbf{u}_{0}, \mathbf{u}_{1}, \ldots, \mathbf{u}_{d}\right) \prod_{\tau=1}^{g}\left(u_{00}+\sum_{\rho=1}^{n} u_{0 \rho} \xi_{\tau \rho}\right)^{(h)},
$$

where $A\left(\mathbf{u}_{0}, \mathbf{u}_{1}, \ldots, \mathbf{u}_{d}\right)$ is in $\mathcal{F}\left\{\mathbf{u}_{0}, \ldots, \mathbf{u}_{d}\right\}, \tilde{\mathbf{u}}=\bigcup_{i=0}^{d} \mathbf{u}_{i} \backslash u_{00}$ and $g=\operatorname{deg}\left(f, u_{00}^{(h)}\right)$. Note that equation (4.8) is formal and should be understood in the following precise meaning: $\left(u_{00}+\sum_{\rho=1}^{n} u_{0 \rho} \xi_{\tau \rho}\right)^{(h)} \triangleq \delta^{h} u_{00}+\delta_{\tau}^{h}\left(\sum_{\rho=1}^{n} u_{0 \rho} \xi_{\tau \rho}\right)$.

Proof. We will follow the notation introduced in the proof of Lemma 4.24. Since $f$ is irreducible, we have $f_{\tau 0}=\left.\frac{\partial f}{\partial u_{00}^{(h)}}\right|_{u_{00}^{(h)}=\gamma_{\tau}} \neq 0$. Let $\xi_{\tau \rho}=f_{\tau \rho} / f_{\tau 0}(\rho=1, \ldots, n)$, where $f_{\tau \rho}=\left.\frac{\partial f}{\partial u_{0 \rho}^{(h)}}\right|_{u_{00}^{(h)}=\gamma_{\tau}}$. Note that $f_{\tau \rho}$ and $\xi_{\tau \rho}$ are in $\mathcal{F}_{\tau}$. We will prove

$$
\gamma_{\tau}=-\delta_{\tau}^{h}\left(u_{01} \xi_{\tau 1}+u_{02} \xi_{\tau 2}+\cdots+u_{0 n} \xi_{\tau n}\right) .
$$

Differentiating the equality $f\left(\mathbf{u} ; \zeta_{0}, \zeta_{1}, \ldots, \zeta_{d}\right)=0$ w.r.t. $u_{0 \rho}^{(h)}$, we have

$$
\overline{\frac{\partial f}{\partial u_{0 \rho}^{(h)}}}+\overline{\frac{\partial f}{\partial u_{00}^{(h)}}}\left(-\xi_{\rho}\right)=0
$$

where $\overline{\frac{\partial f}{\partial u_{0 \rho}^{(h)}}}$ are obtained by substituting $\zeta_{i}$ to $u_{i 0}(i=0,1, \ldots, d)$ in $\frac{\partial f}{\partial u_{0 \rho}^{(h)}}$. Multiplying $u_{0 \rho}$ to the above equation and for $\rho$ from 1 to $n$, adding them together, we have

$$
\sum_{\rho=1}^{n} u_{0 \rho} \frac{\overline{\partial f}}{\partial u_{0 \rho}^{(h)}}+\overline{\frac{\partial f}{\partial u_{00}^{(h)}}}\left(-\sum_{\rho=1}^{n} u_{0 \rho} \xi_{\rho}\right)=\sum_{\rho=1}^{n} u_{0 \rho} \overline{\frac{\partial f}{\partial u_{0 \rho}^{(h)}}}+\zeta_{0} \frac{\overline{\partial f}}{\partial u_{00}^{(h)}}=0 .
$$

Thus, $q=\sum_{\rho=1}^{n} u_{0 \rho} \frac{\partial f}{\partial u_{0 \rho}^{(h)}}+u_{00} \frac{\partial f}{\partial u_{00}^{(h)}} \in \operatorname{sat}(f)$. Since $q$ is of order not greater than $f$, it must be divisible by $f$. Since $q$ and $f$ have the same degree, there exists an $a \in \mathcal{F}$ such that $q=a f$. Setting $u_{00}^{(h)}=\gamma_{\tau}$ on both sides of $q=a f$, we have $\sum_{\rho=1}^{n} u_{0 \rho} f_{\tau \rho}+u_{00} f_{\tau 0}=0$. Hence, as an algebraic equation, we have

$$
u_{00}+\sum_{\rho=1}^{n} u_{0 \rho} \xi_{\tau \rho}=0
$$

under the constraint $u_{00}^{(h)}=\gamma_{\tau}$. Equivalently, the above equation is valid in $\left(\mathcal{F}_{\tau}, \delta_{\tau}\right)$. As a consequence, $\gamma_{\tau}=-\delta_{\tau}^{h}\left(\sum_{\rho=1}^{n} u_{0 \rho} \xi_{\tau \rho}\right)$. Substituting them into equation (4.6), the theorem is proved. 
Remark 4.28. The factors in equation (4.8) are the $h$-th derivatives of the factors in the corresponding factorization of the algebraic Chow form [16, p.37].

For an element $\eta=\left(\eta_{1}, \ldots, \eta_{n}\right)$, denote its truncation up to order $k$ as $\eta^{[k]}=$ $\left(\eta_{1}, \ldots, \eta_{n}, \ldots, \eta_{1}^{(k)}, \ldots, \eta_{n}^{(k)}\right)$.

In the proof of Theorem 4.27 some equations are valid in the algebraic case only. To avoid confusion, we introduce the following notation:

$$
\begin{aligned}
& { }^{a} \mathbb{P}_{0}^{(0)}={ }^{a} \mathbb{P}_{0}:=u_{00}+u_{01} y_{1}+\cdots+u_{0 n} y_{n}, \\
& { }^{a} \mathbb{P}_{0}^{(1)}={ }^{a} \mathbb{P}_{0}^{\prime}:=u_{00}^{\prime}+u_{01}^{\prime} y_{1}+u_{01} y_{1}^{\prime}+\cdots+u_{0 n}^{\prime} y_{n}+u_{0 n} y_{n}^{\prime} \\
& \cdots \\
& { }^{a} \mathbb{P}_{0}^{(s)}:=u_{00}^{(s)}+\sum_{j=1}^{n} \sum_{k=0}^{s}\left(\begin{array}{l}
s \\
k
\end{array}\right) u_{0 j}^{(k)} y_{j}^{(s-k)}
\end{aligned}
$$

which are considered to be algebraic polynomials in $\mathcal{F}\left(\mathbf{u}_{0}^{[s]}, \ldots, \mathbf{u}_{n}^{[s]}\right)\left[\mathbb{Y}^{[s]}\right]$, and $u_{i j}^{(k)}, y_{i}^{(j)}$ are treated as algebraic indeterminates. A point $\eta=\left(\eta_{1}, \ldots, \eta_{n}\right)$ is said to be lying on ${ }^{a} \mathbb{P}_{0}^{(k)}$ if regarded as an algebraic point, $\eta^{[k]}$ is a zero of ${ }^{a} \mathbb{P}_{0}^{(k)}$. As a consequence of (4.9) in the proof of Theorem 4.27, we have

Corollary 4.29. $\left(\xi_{\tau_{1}}, \ldots, \xi_{\tau n}, \ldots, \delta_{\tau}^{(h-1)} \xi_{\tau 1}, \ldots, \delta_{\tau}^{(h-1)} \xi_{\tau n}\right)(\tau=1, \ldots, g)$ are common zeros of ${ }^{a} \mathbb{P}_{0}=0,{ }^{a} \mathbb{P}_{0}^{\prime}=0, \ldots,{ }^{a} \mathbb{P}_{0}^{(h-1)}=0$ where $\delta_{\tau}$ is defined in Lemma 4.24.

Example 4.30. Continue from Example 4.5. In this example, $F(\mathbf{u})=u_{01}^{2}\left(u_{00}^{\prime}\right)^{2}-$ $2 u_{01} u_{01}^{\prime} u_{00} u_{00}^{\prime}+\left(u_{01}^{\prime}\right)^{2} u_{00}^{2}+4 u_{01}^{3} u_{00}$, so $g=2$. Also, $F(\mathbf{u})=u_{01}^{2}\left(u_{00}^{\prime}-u_{01}^{\prime} \frac{u_{00}}{u_{01}}+\right.$ $\left.2 \sqrt{-1} \sqrt{u_{00} u_{01}}\right)\left(u_{00}^{\prime}-u_{01}^{\prime} \frac{u_{00}}{u_{01}}-2 \sqrt{-1} \sqrt{u_{00} u_{01}}\right)$. So we can obtain $\gamma_{\tau}=u_{01}^{\prime} \frac{u_{00}}{u_{01}} \mp$ $2 \sqrt{-1} \sqrt{u_{00} u_{01}}(\tau=1,2)$. Following the proof of Theorem 4.27 we obtain that $\xi_{11}=$ $-u_{00} / u_{01}$, with $u_{00}, u_{01}$ satisfying the relation $\delta_{1} u_{00}=\gamma_{1}=\frac{u_{00}}{u_{01}} u_{01}^{\prime}-2 \sqrt{-1} \sqrt{u_{00} u_{01}}$, and $\xi_{21}=-u_{00} / u_{01}$, with $u_{00}, u_{01}$ satisfying $\delta_{2} u_{00}=\gamma_{2}=\frac{u_{00}}{u_{01}} u_{01}^{\prime}+2 \sqrt{-1} \sqrt{u_{00} u_{01}}$. In other words, $\xi_{11}$ and $\xi_{21}$ are in $\mathcal{F}_{1}$ and $\mathcal{F}_{2}$, respectively. Thus, $F(\mathbf{u})$ has the factorization $F(\mathbf{u})=u_{01}^{2}\left(u_{00}^{\prime}+\xi_{11} u_{01}^{\prime}+2 \sqrt{-1} \sqrt{u_{00} u_{01}}\right)\left(u_{00}^{\prime}+\xi_{21} u_{01}^{\prime}-2 \sqrt{-1} \sqrt{u_{00} u_{01}}\right)=$ $u_{01}^{2}\left(u_{00}+\xi_{11} u_{01}\right)^{\prime}\left(u_{00}+\xi_{21} u_{01}\right)^{\prime}$. Note that both $\xi_{i 1}(i=1,2)$ satisfy ${ }^{a} \mathbb{P}_{0}=$ $u_{00}+u_{01} \xi_{i 1}=0$, but ${ }^{a} \mathbb{P}_{0}^{(1)}=u_{00}^{\prime}+u_{01}^{\prime} \xi_{i 1}+u_{01} \delta_{i} \xi_{i 1} \neq 0$.

Lemma 4.31. In equation (4.8), $A\left(\mathbf{u}_{0}, \mathbf{u}_{1}, \ldots, \mathbf{u}_{d}\right)$ is free of $u_{0 i}^{(h)}(i=1, \ldots, n)$.

Proof. Since $f$ is homogenous in the indeterminates $u_{0 i}$ and its derivatives up to the order $h$, we have

$$
\sum_{\rho=1}^{n} u_{0 \rho}^{(h)} \frac{\partial f}{\partial u_{0 \rho}^{(h)}}+u_{00}^{(h)} \frac{\partial f}{\partial u_{00}^{(h)}}+\sum_{k=0}^{h-1} \sum_{\rho=0}^{n} u_{0 \rho}^{(k)} \frac{\partial f}{\partial u_{0 \rho}^{(k)}}=r f, \quad r \in \mathbb{N} .
$$

In this equation, let $u_{00}^{(h)}=\gamma_{\tau}$. We obtain

$$
\sum_{\rho=1}^{n} u_{0 \rho}^{(h)} f_{\tau \rho}+\gamma_{\tau} f_{\tau 0}+\sum_{k=0}^{h-1} \sum_{\rho=0}^{n} u_{0 \rho}^{(k)} \frac{\widehat{\partial f}}{\partial u_{0 \rho}^{(k)}}=0
$$

where $\frac{\widehat{\partial f}}{\partial u_{0 \rho}^{(k)}}$ means replacing $u_{00}^{(h)}$ by $\gamma_{\tau}$ in $\frac{\partial f}{\partial u_{0 \rho}^{(k)}}$. Consequently,

$$
\gamma_{\tau}=-\sum_{\rho=1}^{n} u_{0 \rho}^{(h)} \xi_{\tau \rho}-\sum_{k=0}^{h-1} \sum_{\rho=0}^{n} u_{0 \rho}^{(k)} \frac{\widehat{\partial f}}{\partial u_{0 \rho}^{(k)}} / f_{\tau 0} .
$$


Hence,

(4.11)

$$
f\left(\mathbf{u} ; u_{00}, u_{10}, \ldots, u_{d 0}\right)=A \prod_{\tau=1}^{g}\left(u_{00}^{(h)}+\sum_{\rho=1}^{n} u_{0 \rho}^{(h)} \xi_{\tau \rho}+\sum_{k=0}^{h-1} \sum_{\rho=0}^{n} u_{0 \rho}^{(k)} \frac{\widehat{\partial f}}{\partial u_{0 \rho}^{(k)}} / f_{\tau 0}\right) .
$$

We claim that $\xi_{\tau \rho}$ and $\sum_{k=0}^{h-1} \sum_{\rho=0}^{n} u_{0 \rho}^{(k)} \frac{\widehat{\partial f}}{\partial u_{0 \rho}^{(k)}} / f_{\tau 0}$ are algebraically independent of $u_{0 i}^{(h)}(i=1, \ldots, n)$. First, since $\xi_{\rho}$ is algebraically independent of $u_{0 i}^{(h)}(i=$ $1, \ldots, n)$, and by Theorem 4.13,

$$
\xi_{\rho}=\frac{\partial f}{\partial u_{0 \rho}^{(h)}} /\left.\frac{\partial f}{\partial u_{00}^{(h)}}\right|_{\left(u_{00}, \ldots, u_{d 0}\right)=\left(\zeta_{0}, \ldots, \zeta_{d}\right)}=\overline{\frac{\partial f}{\partial u_{0 \rho}^{(h)}}} / \overline{\frac{\partial f}{\partial u_{00}^{(h)}}},
$$

we have

$$
\frac{\partial \xi_{\rho}}{\partial u_{0 i}^{(h)}}=\frac{\frac{\partial}{\partial u_{0 i}^{(h)}}\left(\overline{\frac{\partial f}{\partial u_{0 \rho}^{(h)}}}\right) \frac{\overline{\partial f}}{\partial u_{00}^{(h)}}-\frac{\partial}{\partial u_{0 i}^{(h)}}\left(\overline{\frac{\partial f}{\partial u_{00}^{(h)}}}\right) \frac{\overline{\partial f}}{\partial u_{0 \rho}^{(h)}}}{\left(\overline{\frac{\partial f}{\partial u_{00}^{(h)}}}\right)^{2}}=0,
$$

where $\overline{\frac{\partial f}{\partial u_{0 \rho}^{(h)}}}$ is obtained by replacing $\left(u_{00}, \ldots, u_{d 0}\right)$ by $\left(\zeta_{0}, \ldots, \zeta_{d}\right)$ in $\frac{\partial f}{\partial u_{0 \rho}^{(h)}}$, or equivalently,

$$
\frac{\partial}{\partial u_{0 i}^{(h)}}\left(\frac{\partial f}{\partial u_{0 \rho}^{(h)}}\right) \frac{\partial f}{\partial u_{00}^{(h)}}-\frac{\partial}{\partial u_{0 i}^{(h)}}\left(\frac{\partial f}{\partial u_{00}^{(h)}}\right) \frac{\partial f}{\partial u_{0 \rho}^{(h)}} \in \operatorname{sat}(f) .
$$

Set $u_{00}^{(h)}=\gamma_{\tau}$; we have

$$
\frac{\partial}{\partial u_{0 i}^{(h)}}\left(f_{\tau \rho}\right) f_{\tau 0}-\frac{\partial}{\partial u_{0 i}^{(h)}}\left(f_{\tau 0}\right) f_{\tau \rho}=0 .
$$

Thus,

$$
\frac{\partial \xi_{\tau \rho}}{\partial u_{0 i}^{(h)}}=\frac{\partial\left(f_{\tau \rho} / f_{\tau 0}\right)}{\partial u_{0 i}^{(h)}}=0
$$

Second, set $\left(u_{00}, \ldots, u_{d 0}\right)=\left(\zeta_{0}, \ldots, \zeta_{d}\right)$ in the equation

$$
\sum_{\rho=1}^{n} u_{0 \rho}^{(h)} \frac{\partial f}{\partial u_{0 \rho}^{(h)}}+u_{00}^{(h)} \frac{\partial f}{\partial u_{00}^{(h)}}+\sum_{k=0}^{h-1} \sum_{\rho=0}^{n} u_{0 \rho}^{(k)} \frac{\partial f}{\partial u_{0 \rho}^{(k)}}=r f, \quad r \in \mathbb{N} .
$$

We have

$$
\sum_{\rho=1}^{n} u_{0 \rho}^{(h)} \overline{\frac{\partial f}{\partial u_{0 \rho}^{(h)}}}+\zeta_{0}^{(h)} \overline{\frac{\partial f}{\partial u_{00}^{(h)}}}+\overline{\sum_{k=0}^{h-1} \sum_{\rho=0}^{n} u_{0 \rho}^{(k)} \frac{\partial f}{\partial u_{0 \rho}^{(k)}}}=0 .
$$

By Theorem 4.13,

$$
\left[\sum_{\rho=1}^{n} u_{0 \rho}^{(h)} \xi_{\rho}+\zeta_{0}^{(h)}+\overline{\sum_{k=0}^{h-1} \sum_{\rho=0}^{n} u_{0 \rho}^{(k)} \frac{\partial f}{\partial u_{0 \rho}^{(k)}}} / \overline{\frac{\partial f}{\partial u_{00}^{(h)}}}=0 .\right.
$$


Then,

$$
\begin{gathered}
\frac{\partial}{\partial u_{0 i}^{(h)}}\left(\overline{\sum_{k=0}^{h-1} \sum_{\rho=0}^{n} u_{0 \rho}^{(k)} \frac{\partial f}{\partial u_{0 \rho}^{(k)}}} / \overline{\frac{\partial f}{\partial u_{00}^{(h)}}}\right)=-\xi_{i}-\left(-\xi_{i}\right)=0 \\
=\frac{\frac{\partial}{\partial u_{0 i}^{(h)}}\left(\overline{\sum_{k=0}^{h-1} \sum_{\rho=0}^{n} u_{0 \rho}^{(k)} \frac{\partial f}{\partial u_{0 \rho}^{(k)}}}\right) \overline{\frac{\partial f}{\partial u_{00}^{(h)}}}-\frac{\partial}{\partial u_{0 i}^{(h)}}\left(\overline{\frac{\partial f}{\partial u_{00}^{(h)}}}\right)\left(\overline{\sum_{k=0}^{h-1} \sum_{\rho=0}^{n} u_{0 \rho}^{(k)} \frac{\partial f}{\partial u_{0 \rho}^{(k)}}}\right)}{\left(\overline{\frac{\partial f}{\partial u_{00}^{(h)}}}\right)^{2}} .
\end{gathered}
$$

Thus, we have $\frac{\partial}{\partial u_{0 i}^{(h)}}\left(\sum_{k=0}^{h-1} \sum_{\rho=0}^{n} u_{0 \rho}^{(k)} \frac{\partial f}{\partial u_{0 \rho}^{(k)}}\right) \frac{\partial f}{\partial u_{00}^{(h)}}-\frac{\partial}{\partial u_{0 i}^{(h)}}\left(\frac{\partial f}{\partial u_{00}^{(h)}}\right)\left(\sum_{k=0}^{h-1} \sum_{\rho=0}^{n} u_{0 \rho}^{(k)}\right.$ $\left.\frac{\partial f}{\partial u_{0 \rho}^{(k)}}\right) \in \operatorname{sat}(f)$. From Lemma 4.25, by setting $u_{00}^{(h)}=\gamma_{\tau}$ in the above relation, we obtain

$$
\frac{\partial\left(\sum_{k=0}^{h-1} \sum_{\rho=0}^{n} u_{0 \rho}^{(k)} \frac{\widehat{\partial f}}{\partial u_{0 \rho}^{(k)}} / f_{\tau 0}\right)}{\partial u_{0 i}^{(h)}}=0 .
$$

From (4.12) and (4.13), $\xi_{\tau \rho}$ and $\sum_{k=0}^{h-1} \sum_{\rho=0}^{n} u_{0 \rho}^{(k)} \frac{\widehat{\partial f}}{\partial u_{0 \rho}^{(k)}} / f_{\tau 0}$ are algebraically independent of $u_{0 i}^{(h)}(i=1, \ldots, n)$. Then the symmetric functions of $\xi_{\tau 1}, \ldots, \xi_{\tau n}, \sum_{k=0}^{h-1}$ $\sum_{\rho=0}^{n} u_{0 \rho}^{(k)} \frac{\widehat{\partial f}}{\partial u_{0 \rho}^{(k)}} / f_{\tau 0}$ are rational functions in the set of indeterminates $\left\{u_{i k}, \ldots, u_{i k}^{(h)}\right.$, $\left.u_{0 k}, \ldots, u_{0 k}^{(h-1)}: i=1, \ldots, d ; k=0, \ldots, n\right\}$ only. Therefore, $\prod_{\tau=1}^{g}\left(u_{00}^{(h)}-\gamma_{\tau}\right)=\frac{\phi}{\psi}$ where $\psi$ is free of $u_{0 i}^{(h)}(i=1, \ldots, n)$ and $\operatorname{gcd}(\phi, \psi)=1$. Thus $A \phi=f \psi$. Since $f$ is irreducible, we conclude that $A=\psi$ is free of $u_{0 i}^{(h)}(i=1, \ldots, n)$.

Note that the factorization (4.8) is formal in the sense that different factors are from different differential fields $\mathcal{F}_{\tau}$. The following result shows how to obtain a factorization in the same extension field.

Theorem 4.32. The quantities $\xi_{\tau 1}, \ldots, \xi_{\tau n}$ in (4.8) are unique and (4.11) is a factorization of $F$ as an algebraic polynomial in $u_{00}^{(h)}, \ldots, u_{0 n}^{(h)}$ in an extension field of $\mathcal{F}\left(u_{i k}, \ldots, u_{i k}^{(h)}, u_{0 k}, \ldots, u_{0 k}^{(h-1)}: i=1, \ldots, d ; k=0, \ldots, n\right)$.

Proof. From Lemma 4.31, equations (4.12) and (4.13), we can see that $A\left(\mathbf{u}_{0}, \ldots, \mathbf{u}_{d}\right)$, $\xi_{\tau j}$, and $\sum_{k=0}^{h-1} \sum_{\rho=0}^{n} u_{0 \rho}^{(k)} \frac{\widehat{\partial f}}{\partial u_{0 \rho}^{(k)}} / f_{\tau 0}$ are free of $u_{0 i}^{(h)}(i=1, \ldots, n)$. Then, (4.11) is a factorization of the Chow form $F\left(\mathbf{u}_{0}, \ldots, \mathbf{u}_{n}\right)$ in the polynomial ring $\mathcal{F}\left(u_{i k}, \ldots, u_{i k}^{(h)}\right.$, $\left.u_{0 k}, \ldots, u_{0 k}^{(h-1)}: i=1, \ldots, d ; k=0, \ldots, n\right)\left[u_{00}^{(h)}, \ldots, u_{0 n}^{(h)}\right]$. Thus, the factorization (4.11) must be unique, and hence $\xi_{\tau i}$.

4.5. Leading differential degree of an irreducible differential variety. In this subsection, we will define the leading differential degree for a prime $\delta$-ideal and give its geometric meaning.

Definition 4.33. Let $F\left(\mathbf{u}_{0}, \mathbf{u}_{1}, \ldots, \mathbf{u}_{d}\right)=f\left(\mathbf{u} ; u_{00}, \ldots, u_{d 0}\right)$ be the Chow form of a prime $\delta$-F-ideal $\mathcal{I}$ of dimension $d$ and order $h$. By Lemma 4.20, the number $g=\operatorname{deg}\left(f, u_{00}^{(h)}\right)$ is an invariant of $\mathcal{I}$ under invertible linear transformations, which is called the leading differential degree of $\mathcal{I}$. 
From (4.8), there exist $g$ points $\left(\xi_{\tau 1}, \ldots, \xi_{\tau n}\right)(\tau=1, \ldots, g)$, which have interesting properties.

Theorem 4.34. The points $\left(\xi_{\tau 1}, \ldots, \xi_{\tau n}\right)(\tau=1, \ldots, g)$ in (4.8) are generic points of the $\delta$-F-variety $V$. If $d>0$, they also satisfy the equations

$$
u_{\sigma 0}+\sum_{\rho=1}^{n} u_{\sigma \rho} y_{\rho}=0(\sigma=1, \ldots, d) .
$$

Proof. Suppose $\phi\left(y_{1}, \ldots, y_{n}\right) \in \mathcal{F}\{\mathbb{Y}\}$ is any $\delta$-polynomial vanishing on $V$. Then $\phi\left(\xi_{1}, \ldots, \xi_{n}\right)=0$. From Theorem 4.13, $\xi_{\rho}=\overline{\frac{\partial f}{\partial u_{0 \rho}^{(h)}}} / \overline{\frac{\partial f}{\partial u_{00}^{(h)}}}$, we have

$$
\phi\left(\overline{\frac{\partial f}{\partial u_{01}^{(h)}}} / \overline{\frac{\partial f}{\partial u_{00}^{(h)}}}, \ldots, \overline{\frac{\partial f}{\partial u_{0 n}^{(h)}}} / \overline{\frac{\partial f}{\partial u_{00}^{(h)}}}\right)=0,
$$

where $\frac{\overline{\partial f}}{\partial u_{0 \rho}^{(h)}}$ are obtained by substituting $\zeta_{i}$ to $u_{i 0}(i=0,1, \ldots, d)$ in $\frac{\partial f}{\partial u_{0 \rho}^{(h)}}$.

Hence, $\phi\left(\frac{\partial f}{\partial u_{01}^{(h)}} / \frac{\partial f}{\partial u_{00}^{(h)}}, \ldots, \frac{\partial f}{\partial u_{0 n}^{(h)}} / \frac{\partial f}{\partial u_{00}^{(h)}}\right)$ vanishes for $\left(u_{00}, \ldots, u_{d 0}\right)=\left(\zeta_{0}, \ldots, \zeta_{d}\right)$. Then there exists an $m \in \mathbb{N}$ such that $\left(\frac{\partial f}{\partial u_{00}^{(h)}}\right)^{m} \phi\left(\frac{\partial f}{\partial u_{01}^{(h)}} / \frac{\partial f}{\partial u_{00}^{(h)}}, \ldots, \frac{\partial f}{\partial u_{0 n}^{(h)}} / \frac{\partial f}{\partial u_{00}^{(h)}}\right) \in$ $\operatorname{sat}(f)$ in $\mathcal{F}\left\{\tilde{\mathbf{u}}, u_{00}\right\}$. By Lemma 4.25, we have $\left(f_{\tau 0}\right)^{m} \phi\left(\xi_{\tau 1}, \ldots, \xi_{\tau n}\right)=0$. Thus $\phi\left(\xi_{\tau 1}, \ldots, \xi_{\tau n}\right)=0$, which means that $\left(\xi_{\tau 1}, \ldots, \xi_{\tau n}\right) \in V$.

Conversely, for any $p \in \mathcal{F}\{\mathbb{Y}\}$ such that $p\left(\xi_{\tau 1}, \ldots, \xi_{\tau n}\right)=0$, there exists an $l \in \mathbb{N}$ such that $\widetilde{p}=\left(\frac{\partial f}{\partial u_{00}^{(h)}}\right)^{l} p\left(\frac{\partial f}{\partial u_{01}^{(h)}} / \frac{\partial f}{\partial u_{00}^{(h)}}, \ldots, \frac{\partial f}{\partial u_{0 n}^{(h)}} / \frac{\partial f}{\partial u_{00}^{(h)}}\right)$ is in $\mathcal{F}\left\{\tilde{\mathbf{u}}, u_{00}\right\}$, which vanishes at $u_{00}^{(h)}=\gamma_{\tau}$. By Lemma 4.25, $\widetilde{p} \in \operatorname{sat}(f)$. Now treating $\widetilde{p}$ as a $\delta$ polynomial in $\mathcal{F}\langle\mathbf{u}\rangle\left\{u_{00}, \ldots, u_{d 0}\right\}$, we have $\widetilde{p}\left(\xi_{1}, \ldots, \xi_{n}\right)=0$, and hence $p\left(\xi_{1}, \ldots\right.$, $\left.\xi_{n}\right)=0$. So $\left(\xi_{\tau 1}, \ldots, \xi_{\tau n}\right)$ is a generic point of $V$.

Since $\overline{\frac{\partial f}{\partial u_{0 \rho}^{(h)}}}+\overline{\frac{\partial f}{\partial u_{00}^{(h)}}}\left(-\xi_{\rho}\right)=0$, we have $\sum_{\rho=1}^{n} u_{\sigma \rho} \overline{\frac{\partial f}{\partial u_{0 \rho}^{(h)}}}+\zeta_{\sigma} \overline{\frac{\partial f}{\partial u_{00}^{(h)}}}=0$. Thus, $\sum_{\rho=0}^{n} u_{\sigma \rho} \frac{\partial f}{\partial u_{0 \rho}^{(h)}}$ vanishes at $\left(u_{00}, \ldots, u_{d 0}\right)=\left(\zeta_{0}, \ldots, \zeta_{d}\right)$. In the case $\sigma \neq 0, \sum_{\rho=0}^{n} u_{\sigma \rho}$ $\frac{\partial f}{\partial u_{0 \rho}^{(h)}}=0$. Consequently, $u_{\sigma 0}+\sum_{\rho=1}^{n} u_{\sigma \rho} \xi_{\tau \rho}=0(\sigma=1, \ldots, d)$.

Example 4.35. Continue from Example 4.30. We have $\xi_{11}=-u_{00} / u_{01}$ under the condition $u_{00}^{\prime}=\gamma_{1}=\frac{u_{00}}{u_{01}} u_{01}^{\prime}-2 \sqrt{-1} \sqrt{u_{00} u_{01}}$. Then $\xi_{11}^{\prime}=2 \sqrt{-\frac{u_{00}}{u_{01}}}$ and $\xi_{11}$ is a zero of the original $\delta$-ideal $\operatorname{sat}\left(y_{1}^{\prime 2}-4 y_{1}\right)$.

Now, we will prove a result which gives the geometrical meaning of the leading $\delta$-degree.

Suppose $F\left(\mathbf{u}_{0}, \ldots, \mathbf{u}_{d}\right)$ is the Chow form of $V$ which is of dimension $d$, order $h$, and leading differential degree $g$. Recall that by saying a point $\eta=\left(\eta_{1}, \ldots, \eta_{n}\right)$ lying on ${ }^{a} \mathbb{P}_{0}^{(k)}$ we mean that $\eta^{[k]}=\left(\eta_{1}, \ldots, \eta_{n}, \ldots, \eta_{1}^{(k)}, \ldots, \eta_{n}^{(k)}\right)$ is an algebraic zero of ${ }^{a} \mathbb{P}_{0}^{(k)}$. Theorem 4.34 and Corollary 4.29 show that $\left(\xi_{\tau 1}, \ldots, \xi_{\tau n}\right)(\tau=1, \ldots, g)$ are intersection points of $V$ and $\mathbb{P}_{i}=0(i=1, \ldots, d)$ as well as ${ }^{a} \mathbb{P}_{0}^{(k)}=0(k=$ $0, \ldots, h-1)$. In the next theorem, we will prove the converse of this result, that is, $\left(\xi_{\tau 1}, \ldots, \xi_{\tau n}\right)(\tau=1, \ldots, g)$ are the only elements in $V$ which are also on $\mathbb{P}_{i}=0(i=$ $1, \ldots, d)$ as well as on ${ }^{a} \mathbb{P}_{0}^{(k)}=0(k=0, \ldots, h-1)$. Intuitively, we use $\mathbb{P}_{i}=0(i=$ $1, \ldots, d)$ to decrease the dimension of $V$ to zero and use ${ }^{a} \mathbb{P}_{0}^{(k)}=0(k=0, \ldots, h-1)$ 
to determine the $h$ arbitrary constants in the solutions of the zero-dimensional $\delta$-variety.

Theorem 4.36. $\left(\xi_{\tau 1}, \ldots, \xi_{\tau n}\right)(\tau=1, \ldots, g)$ defined in (4.8) are the only elements of $V$ which also lie on $\mathbb{P}_{i}(i=1, \ldots, d)^{3}$ as well as on ${ }^{a} \mathbb{P}_{0}^{(j)}(j=0, \ldots, h-1)$.

Proof. First, by Theorem 4.34 and Corollary 4.29, $\left(\xi_{\tau 1}, \ldots, \xi_{\tau n}\right)(\tau=1, \ldots, g)$ are solutions of $\mathbb{I}(V)$ and $\mathbb{P}_{1}, \ldots, \mathbb{P}_{d}$, which also lie on ${ }^{a} \mathbb{P}_{0},{ }^{a} \mathbb{P}_{0}^{\prime}, \ldots,{ }^{a} \mathbb{P}_{0}^{(h-1)}$. It suffices to show that the number of solutions of $\mathbb{I}(V)$ and $\mathbb{P}_{1}, \ldots, \mathbb{P}_{d}$, which also lie on ${ }^{a} \mathbb{P}_{0},{ }^{a} \mathbb{P}_{0}^{\prime}, \ldots,{ }^{a} \mathbb{P}_{0}^{(h-1)}$, does not exceed $g$.

Let $\mathcal{J}=\left[\mathbb{I}(V), \mathbb{P}_{1}, \ldots, \mathbb{P}_{d}\right] \subset \mathcal{F}^{\star}\{\mathbb{Y}\}$, where $\mathcal{F}^{\star}=\mathcal{F}\left\langle\bigcup_{i=1}^{d} \mathbf{u}_{i}\right\rangle$. By Theorem 3.14. $\mathcal{J}$ is a prime $\delta$-ideal of dimension zero and order $h$. Let $\mathcal{J}^{\langle h\rangle}=\mathcal{J} \cap \mathcal{F}^{\star}\left[\mathbb{Y}^{[h]}\right]$. Since $\mathcal{J}$ is of dimension zero and order $h$, its $\delta$-dimension polynomial is of the form $\omega(t)=h$, for $t \geq h$. So $\mathcal{J}^{\langle h\rangle}$ is an algebraic prime ideal of dimension $h$.

Let $\mathcal{J}_{0}=\left(\mathcal{J}^{\langle h\rangle},{ }^{a} \mathbb{P}_{0},{ }^{a} \mathbb{P}_{0}^{\prime}, \ldots,{ }^{a} \mathbb{P}_{0}^{(h-1)}\right)$ be an algebraic ideal in the polynomial $\operatorname{ring} \mathcal{F}_{0}\left[\mathbb{Y}^{[h]}, u_{00}, \ldots, u_{00}^{(h-1)}\right]$, where $\mathcal{F}_{0}=\mathcal{F}^{\star}\left(\bigcup_{j=1}^{n} u_{0 j}^{[h-1]}\right)$ and $u_{0 j}^{[h-1]}$ denote the set $\left\{u_{0 j}, u_{0 j}^{\prime}, \ldots, u_{0 j}^{(h-1)}\right\}$. Similar to the proof of Lemma 3.5. we can show that $\mathcal{J}_{0}$ is an algebraic prime ideal of dimension $h$. If we can prove that $u_{00}^{[h-1]}=\left\{u_{00}, \ldots, u_{00}^{(h-1)}\right\}$ is a parametric set of $\mathcal{J}_{0}$, then it is clear that $\mathcal{J}_{1}=\left(\mathcal{J}_{0}\right)$ is an algebraic prime ideal of dimension zero in $\mathcal{F}_{0}\left(u_{00}^{[h-1]}\right)\left[\mathbb{Y}^{[h]}\right]=\mathcal{F}^{*}\left(\mathbf{u}_{0}^{[h-1]}\right)\left[\mathbb{Y}^{[h]}\right]$. So we need to prove that $u_{00}^{[h-1]}$ is a parametric set of $\mathcal{J}_{0}$. Suppose the contrary. Then there exists a nonzero $\delta$-polynomial involving only $\left\{u_{00}, \ldots, u_{00}^{(h-1)}\right\}$ as well as the other $u$, which belongs to $\mathcal{J}_{0}$. Such a $\delta$-polynomial also belongs to $\left[\mathcal{J}, \mathbb{P}_{0}\right] \in \mathcal{F}^{\star}\left\langle u_{01}, \ldots, u_{0 n}\right\rangle\left\{y_{1}, \ldots, y_{n}\right.$, $\left.u_{00}\right\}$. From the proof of Theorem 4.11. $\left\{F, S_{F} y_{1}-\frac{\partial F}{\partial u_{01}^{(s)}}, \ldots, S_{F} y_{n}-\frac{\partial F}{\partial u_{0 n}^{(s)}}\right\}$ is a characteristic set of $\left[\mathcal{J}, \mathbb{P}_{0}\right]$ w.r.t. the elimination ranking $u_{00} \prec y_{1} \prec \cdots \prec y_{n}$. So this $\delta$-polynomial can be reduced to zero by $F\left(\mathbf{u}_{0}, \ldots, \mathbf{u}_{d}\right)$. But $\operatorname{ord}\left(F, u_{00}\right)=h$, a contradiction. So we have proved that $\mathcal{J}_{1}$ is an algebraic prime ideal of dimension zero.

Clearly, $\mathcal{J}_{2}=\left(\mathcal{J}_{1},{ }^{a} \mathbb{P}_{0}^{(h)}\right) \in \mathcal{F}_{0}\left(u_{00}^{[h-1]}, \cup_{j=1}^{n} u_{0 j}^{[h]}\right)\left[\mathbb{Y}^{[h]}, u_{00}^{(h)}\right]$ is an algebraic prime ideal of dimension zero. Then, there exists an irreducible polynomial involving only $u_{i j}^{(k)}$ and $u_{00}^{(h)}$. Similar to above, it also belongs to $\left[\mathcal{J}, \mathbb{P}_{0}\right]$, thus it can be divisible by $F$. Since $F$ is irreducible, it differs from $F$ only by a factor in $\mathcal{F}$. Thus, $F=f\left(\mathbf{u} ; u_{00}, u_{10}, \ldots, u_{d 0}\right) \in \mathcal{J}_{2}$.

Let $\left(\xi_{1}, \ldots, \xi_{n}\right)$ be a generic point of $V$ and $\zeta_{i}=-\sum_{j=1}^{n} u_{i j} \xi_{j}(i=0, \ldots, d)$. By Lemma 4.3 the $\delta$-ideal $\left[\mathbb{I}(V), \mathbb{P}_{1}, \ldots, \mathbb{P}_{d}, \mathbb{P}_{0}\right]$ in $\mathcal{F}\langle\mathbf{u}\rangle\left\{y_{1}, \ldots, y_{n}, u_{00}, \ldots, u_{d 0}\right\}$ has a generic point $\left(\xi_{1}, \ldots, \xi_{n}, \zeta_{0}, \ldots, \zeta_{d}\right)$. Since $f\left(\mathbf{u} ; \zeta_{0}, \ldots, \zeta_{d}\right)=0$, differentiating both sides of this identity w.r.t. $u_{0 j}^{(k)}$, we have the following identities:

$$
\overline{\frac{\partial f}{\partial u_{0 j}^{(k)}}}+\sum_{l=k}^{h} \frac{\overline{\partial f}}{\partial u_{00}^{(l)}}\left(-\left(\begin{array}{l}
l \\
k
\end{array}\right) \xi_{j}^{(l-k)}\right)=0, \quad(j=1, \ldots, n ; k=0, \ldots, h),
$$

where $\overline{\frac{\partial f}{\partial u_{0 i}^{(j)}}}$ are obtained by substituting $\zeta_{i}$ to $u_{i 0}$ in $\frac{\partial f}{\partial u_{0 i}^{(j)}}$. Let $g_{j k}=\left(\begin{array}{l}h \\ k\end{array}\right) \frac{\partial f}{\partial u_{00}^{(h)}} y_{j}^{(k)}+$ $\sum_{l=1}^{k}\left(\begin{array}{l}h-l \\ k-l\end{array}\right) \frac{\partial f}{\partial u_{00}^{(h-l)}} y_{j}^{(k-l)}-\frac{\partial f}{\partial u_{0 j}^{(h-k)}}(j=1, \ldots, n ; k=0, \ldots, h)$. Then $g_{j k} \in\left[\mathbb{I}(V), \mathbb{P}_{1}\right.$,

\footnotetext{
${ }^{3}$ If $d=0, \mathbb{P}_{i}(i=1, \ldots, d)$ is empty.
} 
$\left.\ldots, \mathbb{P}_{d}, \mathbb{P}_{0}\right] \subset\left[\mathcal{J}, \mathbb{P}_{0}\right]$, for $g_{j k}$ vanishes at $\left(\xi_{1}, \ldots, \xi_{n}, \zeta_{0}, \ldots, \zeta_{d}\right)$. Denote the algebraic ideal $\left[\mathcal{J}, \mathbb{P}_{0}\right] \cap \mathcal{F}^{\star}\left(\bigcup_{j=1}^{n} u_{0 j}^{[h]}\right)\left[\mathbb{Y}^{[h]}, u_{00}, \ldots, u_{00}^{(h)}\right]$ by $\left[\mathcal{J}, \mathbb{P}_{0}\right]^{\langle h\rangle}$. It is clear that $g_{j k} \in\left[\mathcal{J}, \mathbb{P}_{0}\right]^{\langle h\rangle}$. We will show that $\left[\mathcal{J}, \mathbb{P}_{0}\right]^{\langle h\rangle}=\left(\mathcal{J}\langle h\rangle,{ }^{a} \mathbb{P}_{0}, \ldots,{ }^{a} \mathbb{P}_{0}^{(h)}\right)$, which implies that $g_{j k} \in \mathcal{J}_{2}$. Let $\eta=\left(\eta_{1}, \ldots, \eta_{n}\right)$ be a generic point of $\mathcal{J}$. Then $\left(\eta_{1}, \ldots, \eta_{n}\right.$, $\left.-\sum_{j=1}^{n} u_{0 j} \eta_{j}\right)$ is a generic point of $\left[\mathcal{J}, \mathbb{P}_{0}\right]$. Thus,

$$
\left(\eta_{1}, \ldots, \eta_{n}, \ldots, \eta_{1}^{(h)}, \ldots, \eta_{n}^{(h)},-\sum_{j=1}^{n} u_{0 j} \eta_{j}, \ldots,-\sum_{j=1}^{n}\left(u_{0 j} \eta_{j}\right)^{(h)}\right)
$$

is a generic point of $\left[\mathcal{J}, \mathbb{P}_{0}\right]^{\langle h\rangle}$. Of course, it is also a generic point of $\left(\mathcal{J}^{\langle h\rangle},{ }^{a} \mathbb{P}_{0}\right.$, $\left.\ldots,{ }^{a} \mathbb{P}_{0}^{(h)}\right)$. So the two ideals are identical. Thus, $g_{j k}$ belongs to $\mathcal{J}_{2}$. Note that the coefficient of $y_{j}^{(k)}$ in $g_{j k}$ is $\left(\begin{array}{l}h \\ k\end{array}\right) S_{f}=\left(\begin{array}{l}h \\ k\end{array}\right) \frac{\partial f}{\partial u_{00}^{(h)}}$. So $\mathbb{V}\left(\mathcal{J}_{2}\right) \subseteq \mathbb{V}\left(f\left(\mathbb{U}_{0}, u_{00}^{(h)}\right), g_{j k}\right.$ : $j=1, \ldots, n ; k=0, \ldots, h)$, and the latter algebraic variety consists of exactly $g$ elements. Thus, $\left|\mathbb{V}\left(\mathcal{J}_{1}\right)\right|=\left|\mathbb{V}\left(\mathcal{J}_{2}\right)\right| \leq g$. Since every solution of $\mathcal{J}$ which also lies on ${ }^{a} \mathbb{P}_{0},{ }^{a} \mathbb{P}_{0}^{\prime}, \ldots,{ }^{a} \mathbb{P}_{0}^{(h-1)}$, when truncated up to order $h$, becomes a solution of $\mathcal{J}_{1}$, it follows that the number of solutions of $\mathbb{I}(V)$ and $\mathbb{P}_{1}, \ldots, \mathbb{P}_{d}$ which also lie on ${ }^{a} \mathbb{P}_{0}, \mathbb{P}_{0}^{\prime}, \ldots,{ }^{a} \mathbb{P}_{0}^{(h-1)}$ does not exceed $g$.

With Theorems 4.27, 4.32, 4.34, and 4.36, we proved the second and third statements of Theorem 1.2 .

From the proof, we can see that a zero-dimensional algebraic ideal is obtained as shown by the following corollary.

Corollary 4.37. Let $\mathcal{I}$ be a prime $\delta$-ideal in $\mathcal{F}\{\mathbb{Y}\}$ of dimension $d$, order $h$, and leading $\delta$-degree $g$. Use the same notation as Theorem 4.36. Then

$$
\widetilde{\mathcal{I}}=\left(\left[\mathcal{I}, \mathbb{P}_{1}, \ldots, \mathbb{P}_{d}\right] \cap \mathcal{F}^{\star}\left[\mathbb{Y}^{[h]}\right],{ }^{a} \mathbb{P}_{0}, \ldots,{ }^{a} \mathbb{P}_{0}^{(h-1)}\right) \subset \mathcal{F}^{\star}\left(\mathbf{u}_{0}^{[h-1]}\right)\left[\mathbb{Y}^{[h]}\right]
$$

is an algebraic prime ideal of dimension zero whose solutions are exactly $\left(\xi_{\tau 1}, \ldots\right.$, $\left.\xi_{\tau n}\right)^{[h]}(\tau=1, \ldots, g)$, where $\mathcal{F}^{\star}=\mathcal{F}\left\langle\bigcup_{i=1}^{d} \mathbf{u}_{i}\right\rangle$.

Example 4.38. Continue from Example 4.30. Note that $d=0, h=1$, and $g=2$. Let $V$ be the general component of $p=y_{1}^{\prime 2}-4 y_{1}=0$. As in Theorem 4.36. we introduce the equation ${ }^{a} \mathbb{P}_{0}=u_{00}+u_{01} y_{1}$ which intersects $V$ at two points: $\xi_{11}=-u_{00} / u_{01}$, with $\delta_{1} \xi_{11}=2 \sqrt{-u_{00} / u_{01}}$, and $\xi_{21}=-u_{00} / u_{01}$, with $\delta_{2} \xi_{21}=-2 \sqrt{-u_{00} / u_{01}}$. As indicated by Corollary 4.37 $\left(\xi_{11}, \delta_{1} \xi_{11}\right)$ and $\left(\xi_{21}, \delta_{2} \xi_{21}\right)$ are the only solutions of the algebraic ideal $\widetilde{\mathcal{I}}=\left(p,{ }^{a} \mathbb{P}_{0}\right) \subset \mathbb{Q}\left(u_{00}, u_{01}\right)\left[y_{1}, y_{1}^{\prime}\right]$.

Due to Corollary 4.37, we can give a differential analog to Stickelberger's Theorem in algebraic geometry.

Theorem 4.39 (Stickelberger's Theorem [9, p.54], [33]). Let $\mathcal{P} \subset \mathcal{F}[\mathbb{Y}]$ be a zerodimensional ideal. Denote $A=\mathcal{F}[\mathbb{Y}] / \mathcal{P}$. Then $A$ is a finite-dimensional vector space over $\mathcal{F}$. For any polynomial $f \in \mathcal{F}[\mathbb{Y}]$, let $L_{f}$ be the $\mathcal{F}$-linear map:

$$
L_{f}: \quad \begin{aligned}
& A \\
& \bar{g}
\end{aligned} \frac{A}{f g}
$$

where $\bar{g}$ denotes the residue class of $g \in \mathcal{F}[\mathbb{Y}]$ in $A=\mathcal{F}[\mathbb{Y}] / \mathcal{P}$. Then the eigenvalues of $L_{f}$ are $f(\alpha)$, with multiplicity $m_{\alpha}$, where $\alpha \in \mathbb{V}(\mathcal{P})$ and $m_{\alpha}$ is the multiplicity of $\alpha \in \mathbb{V}(\mathcal{P})$. Thus, the determinant of $L_{f}$ is $\prod_{\alpha \in \mathbb{V}(\mathcal{P})} f(\alpha)^{m_{\alpha}}$. 
Theorem 4.40 (Differential Stickelberger's Theorem). Let $\mathcal{I}$ be a prime $\delta$-ideal in $\mathcal{F}\{\mathbb{Y}\}$ of dimension zero, order $h$, and leading $\delta$-degree $g$. Let $\mathbb{P}_{0}=u_{00}+u_{01} y_{1}+$ $\cdots+u_{0 n} y_{n}=0$ be a generic $\delta$-hyperplane and ${ }^{a} \mathbb{P}_{0}, \ldots,{ }^{a} \mathbb{P}_{0}^{(h-1)}$ be defined as in (4.10). For any $\delta$-polynomial $p \in \mathcal{F}\{\mathbb{Y}\}$, let $s=\max \{h, \operatorname{ord}(p)\}$ and $L_{p}$ be the $\mathcal{F}\left(\mathbf{u}_{0}^{[h-1]}\right)$-linear map:

$$
L_{p}: \quad \mathcal{F}\left(\mathbf{u}_{0}^{[h-1]}\right)\left[\mathbb{Y}^{[s]}\right] / \widetilde{\mathcal{I}} \longrightarrow \mathcal{F}\left(\mathbf{u}_{0}^{[h-1]}\right)\left[\mathbb{Y}^{[s]}\right] / \widetilde{\mathcal{I}}
$$

where $\tilde{\mathcal{I}}=\left(\mathcal{I} \cap \mathcal{F}\left[\mathbb{Y}^{[s]}\right],{ }^{a} \mathbb{P}_{0}, \ldots,{ }^{a} \mathbb{P}_{0}^{(h-1)}\right) \subset \mathcal{F}\left(\mathbf{u}_{0}^{[h-1]}\right)\left[\mathbb{Y}^{[s]}\right]$ and $\bar{g}$ denotes the residue class of $g \in \mathcal{F}\left(\mathbf{u}_{0}^{[h-1]}\right)\left[\mathbb{Y}^{[s]}\right]$ in $\mathcal{F}\left(\mathbf{u}_{0}^{[h-1]}\right)\left[Y^{[s]}\right] / \widetilde{\mathcal{I}}$. Then the eigenvalues of $L_{p}$ are $p\left(\xi_{\tau}\right)$ and the determinant of $L_{p}$ is $\prod_{\tau=1}^{g} p\left(\xi_{\tau}\right)$.

Proof. By Theorem 4.39, it suffices to show that $\widetilde{\mathcal{I}}$ is a prime $\delta$-ideal in $\mathcal{F}\left(\mathbf{u}_{0}^{[h-1]}\right)\left[\mathbb{Y}^{[s]}\right]$ of dimension zero, and $\mathbb{V}(\widetilde{\mathcal{I}})=\left\{\xi_{\tau}^{[s]}=\left(\xi_{\tau 1}, \ldots, \xi_{\tau n}, \ldots, \delta_{\tau}^{s} \xi_{\tau 1}, \ldots, \delta_{\tau}^{s} \xi_{\tau n}\right): \tau=\right.$ $1, \ldots, g\}$. If $s=h$, then this is a direct consequence of Corollary 4.37 If $s>h$, $\xi_{\tau}^{[s]}$ clearly vanishes ${ }^{a} \mathbb{P}_{0}^{(i)}(i=0, \ldots, h-1)$. By Theorem 4.36, $\xi_{\tau}^{[s]}$ are also zeros of $\mathcal{I} \cap \mathcal{F}\left[\mathbb{Y}^{[s]}\right]$ and are the only zeros of $\widetilde{\mathcal{I}}$.

4.6. Relations between the differential Chow form and the variety. In the algebraic case, we can obtain the defining equations of a variety from its Chow form. But in the differential case, this is not valid. Now we proceed as follows to obtain a weaker result. Recall that a $\delta$-variety is unmixed if all of its components have the same dimension.

Lemma 4.41. Let $V$ be an irreducible $\delta$-F -variety of dimension $d>0$ and $(0, \ldots, 0)$ $\notin V$. Then, the intersection of $V$ with a generic $\delta$-hyperplane passing through $(0, \ldots, 0)$ is either empty or unmixed of dimension $d-1$. Moreover, in the case $d>1$, it is exactly unmixed of dimension $d-1$.

Proof. Let $\mathcal{I}=\mathbb{I}(V)$ be the prime $\delta$ - $\mathcal{F}$-ideal corresponding to $V$. A generic $\delta$ hyperplane passing through $(0, \ldots, 0)$ is $u_{1} y_{1}+u_{2} y_{2}+\cdots+u_{n} y_{n}$, where the $u_{i} \in \mathcal{E}$ are $\delta$ - $\mathcal{F}$-indeterminates. Since $(0,0, \ldots, 0) \notin V$, we have

$$
\begin{aligned}
& V \cap \mathbb{V}\left(u_{1} y_{1}+u_{2} y_{2}+\cdots+u_{n} y_{n}\right) \\
= & \mathbb{V}\left(\mathcal{I}, u_{1} y_{1}+u_{2} y_{2}+\cdots+u_{n} y_{n}\right) \\
= & \bigcup_{i=1}^{n} \mathbb{V}\left(\left[\mathcal{I}, u_{1} y_{1}+u_{2} y_{2}+\cdots+u_{n} y_{n}\right] / y_{i}\right) \\
= & \bigcup_{i=1}^{n} \mathbb{V}\left(\left[\mathcal{I}, u_{1} y_{1}+u_{2} y_{2}+\cdots+u_{n} y_{n}\right]: y_{i}^{\infty}\right) .
\end{aligned}
$$

Suppose a generic point of $V$ is $\left(\xi_{1}, \ldots, \xi_{n}\right)$. Since $(0,0, \ldots, 0) \notin V$, there exists at least one $i \in\{1, \ldots, n\}$ such that $\xi_{i} \neq 0$. Of course, $\xi_{i}=0$ means $\mathbb{V}\left(\left[\mathcal{I}, u_{1} y_{1}+\right.\right.$ $\left.\left.u_{2} y_{2}+\cdots+u_{n} y_{n}\right]: y_{i}^{\infty}\right)=\emptyset$. So we need only consider the case when $\xi_{i} \neq 0$. Without loss of generality, we suppose $\xi_{1} \neq 0$.

Let

$$
\mathcal{Q}=\left[\mathcal{I}, u_{1} y_{1}+u_{2} y_{2}+\cdots+u_{n} y_{n}\right]: y_{1}^{\infty} \subseteq \mathcal{F}\left\langle u_{1}, \ldots, u_{n}\right\rangle\left\{y_{1}, \ldots, y_{n}\right\}
$$

and

$$
\mathcal{Q}_{0}=\left[\mathcal{I}, u_{1} y_{1}+u_{2} y_{2}+\cdots+u_{n} y_{n}\right]: y_{1}^{\infty} \subseteq \mathcal{F}\left\langle u_{2}, \ldots, u_{n}\right\rangle\left\{y_{1}, \ldots, y_{n}, u_{1}\right\} .
$$


It is easy to verify that $\left(\xi_{1}, \ldots, \xi_{n},-\frac{u_{2} \xi_{2}+\cdots+u_{n} \xi_{n}}{\xi_{1}}\right)$ is a generic point of $\mathcal{Q}_{0}$ and $\operatorname{dim}\left(\mathcal{Q}_{0}\right)=d$ by following the proof of Lemma 3.5. Now we discuss it in three cases.

Case 1. $\mathcal{I} \cap \mathcal{F}\left\{y_{1}\right\} \neq\{0\}$, that is, $\xi_{1}$ is $\delta$-algebraic over $\mathcal{F}$. We have

$$
\operatorname{dim} V=d=\text { d.tr.deg } \mathcal{F}\left\langle\xi_{1}, \ldots, \xi_{n}\right\rangle / \mathcal{F}=\text { d.tr.deg } \mathcal{F}\left\langle\xi_{1}\right\rangle\left\langle\xi_{2}, \ldots, \xi_{n}\right\rangle / \mathcal{F}\left\langle\xi_{1}\right\rangle .
$$

Suppose $\xi_{2}, \ldots, \xi_{d+1}$ are $\delta$-independent over $\mathcal{F}\left\langle\xi_{1}\right\rangle$.

First, $\mathcal{Q}_{0} \cap \mathcal{F}\left\langle u_{2}, \ldots, u_{n}\right\rangle\left\{u_{1}\right\}=\{0\}$. For if not, we have a nonzero $\delta$-polynomial $h\left(u_{2}, \ldots, u_{n}, u_{1}\right) \in \mathcal{F}\left\{u_{2}, \ldots, u_{n}, u_{1}\right\}$ such that $h\left(u_{2}, \ldots, u_{n},-\frac{u_{2} \xi_{2}+\cdots+u_{n} \xi_{n}}{\xi_{1}}\right)=0$. For a fixed $i$ between 2 and $n$, if we specialize $u_{i}$ to -1 and $u_{j}(j \neq i)$ to 0 , then by Theorem 2.16, $\xi_{i} / \xi_{1}$ is $\delta$-algebraic over $\mathcal{F}$. So each $\xi_{i}(i=1, \ldots, n)$ is $\delta$-algebraic over $\mathcal{F}$, which contradicts the fact that $d>0$. It follows that $\mathcal{Q}$ is not the unit ideal and $\operatorname{dim}(\mathcal{Q}) \geq 0$.

Second, since $y_{2}, \ldots, y_{d+1}$ is a parametric set of $\mathcal{I}$, it is also a parametric set for $\mathcal{Q}_{0}$. So $y_{2}, \ldots, y_{d+1}, u_{1}$ are $\delta$-dependent modulo $\mathcal{Q}_{0}$. Since $\mathcal{Q}_{0} \cap \mathcal{F}\left\langle u_{2}, \ldots, u_{n}\right\rangle\left\{u_{1}\right\}=$ $\{0\}$, we know that $y_{2}, \ldots, y_{d+1}$ are $\delta$-dependent modulo $\mathcal{Q}$. Using the fact that each remaining $y_{i}$ and $y_{2}, \ldots, y_{d+1}$ are $\delta$-dependent modulo $Q$, we obtain $\operatorname{dim}(\mathcal{Q}) \leq d-1$. If $d=1$, then $\operatorname{dim}(\mathcal{Q})=0=d-1$ follows. Now for $d>1$, we claim that $\operatorname{dim}(\mathcal{Q})=d-1$ by proving that $y_{2}, \ldots, y_{d}$ are $\delta$-independent modulo $\mathcal{Q}$. For if not, there exists $0 \neq h\left(y_{2}, \ldots, y_{d}, u_{1}\right) \in \mathcal{Q}_{0}$ such that $h\left(\xi_{2}, \ldots, \xi_{d},-\frac{u_{2} \xi_{2}+\cdots+u_{n} \xi_{n}}{\xi_{1}}\right)=0$. By Theorem 2.16, we can specialize $u_{d+1}$ to -1 , the other $u_{i}$ to zero, and conclude that $\xi_{2}, \ldots, \xi_{d}, \frac{\xi_{d+1}}{\xi_{1}}$ are $\delta$-dependent over $\mathcal{F}$. Since $\xi_{2}, \ldots, \xi_{d}$ are $\delta$-independent over $\mathcal{F}, \xi_{d+1}$ is $\delta$-algebraic over $\mathcal{F}\left\langle\xi_{1}, \ldots, \xi_{d}\right\rangle$, which is a contradiction. Thus $\operatorname{dim} \mathcal{Q}=d-1$.

Case 2. $d>1$ and $\xi_{1}$ is $\delta$-transcendental over $\mathcal{F}$. In this case, we suppose a $\delta$-transcendence basis is $\xi_{1}, \ldots, \xi_{d}$.

First, $\mathcal{Q}_{0} \cap \mathcal{F}\left\langle u_{2}, \ldots, u_{n}\right\rangle\left\{u_{1}\right\}=\{0\}$. For if not, as in the preceding case, we conclude that $\xi_{i} / \xi_{1}$ is $\delta$-algebraic over $\mathcal{F}$, that is, $\xi_{i}, \xi_{1}$ are $\delta$-algebraic over $\mathcal{F}$, which contradicts the fact that $d>1$. So $\mathcal{Q}$ is a nontrivial prime $\delta$-ideal.

Second, $\operatorname{dim}(\mathcal{Q})=d-1$, for on the one hand from the fact that $y_{1}, y_{2}, \ldots, y_{d}, u_{1}$ are $\delta$-dependent modulo $\mathcal{Q}_{0}$, we have $\operatorname{dim}(\mathcal{Q}) \leq d-1$, and on the other hand, from the fact that $y_{2}, \ldots, y_{d}, u_{1}$ are $\delta$-independent modulo $\mathcal{Q}_{0}$, there comes $\operatorname{dim}(\mathcal{Q}) \geq$ $d-1$.

Case 3. $d=1$ and $\xi_{1}$ is $\delta$-transcendental over $\mathcal{F}$. If $\mathcal{Q}_{0} \cap \mathcal{F}\left\langle u_{2}, \ldots, u_{n}\right\rangle\left\{u_{1}\right\} \neq\{0\}$, the intersection is empty. If $\mathcal{Q} \neq[1]$, similar to Case 2 , we can easily prove that the intersection is of dimension zero.

So for each $i \in\{1, \ldots, n\}$ such that $\xi_{i} \neq 0$, we can show that $\mathbb{V}\left(\left[\mathcal{I}, u_{1} y_{1}+u_{2} y_{2}+\right.\right.$ $\left.\left.\cdots+u_{n} y_{n}\right]: y_{i}^{\infty}\right)$ is either empty or of dimension $d-1$, similar to the above steps for the case $i=1$. If $d>1$, it is exactly of dimension $d-1$. Thus the theorem is proved.

By saying independent generic $\delta$-hyperplanes, we mean that the coefficients of these $\delta$-hyperplanes are $\delta$-indedeterminates in $\mathcal{E}$. The following result gives an equivalent condition for a point to be in a $\delta$-variety.

Theorem 4.42. Let $V$ be a $\delta$-F-variety of dimension $d$. Then $\bar{x} \in V$ if and only if $d+1$ independent generic $\delta$-hyperplanes $\mathbb{P}_{0}, \mathbb{P}_{1}, \ldots, \mathbb{P}_{d}$ passing through $\bar{x}$ meet $V$. 
Proof. The necessity of the condition is obviously true. We now consider the sufficiency. We adjoin the coordinates of $\bar{x}$ to $\mathcal{F}$, and denote $\overline{\mathcal{F}}$ to be the $\delta$-field thus obtained. Regarded as a $\delta$-variety over $\overline{\mathcal{F}}, V$ is the sum of a finite number of irreducible $\delta$-varieties $\bar{V}_{i}$, which are of dimension $d$ [32, p.51]. Suppose $\bar{x} \notin V$, and therefore does not lie in any component of $V$. We now prove that any $d+1$ independent generic $\delta$-hyperplanes passing through $\bar{x}$ do not meet $\bar{V}_{i}$. Without loss of generality, suppose $\bar{x}=(0,0, \ldots, 0)$. Then a generic $\delta$-hyperplane passing through $\bar{x}$ is $s_{1} y_{1}+\cdots+s_{n} y_{n}$, where $s_{i} \in \mathcal{E}$ are $\delta$ - $\mathcal{F}$-indeterminates. We proceed by induction on $d$.

If $d=0$, then for $\left(a_{1}, \ldots, a_{n}\right) \in V$, each $a_{i}$ is $\delta$-algebraic over $\mathcal{F}$. If $V \cap \mathbb{V}\left(s_{1} y_{1}+\right.$ $\left.\cdots+s_{n} y_{n}\right) \neq \emptyset$, then there exists some $\left(a_{1}, \ldots, a_{n}\right) \in V$ such that $s_{1} a_{1}+\cdots+$ $s_{n} a_{n}=0$, since the $s_{i}$ are $\delta$-independent over $\mathcal{F}$. Thus $\left(a_{1}, \ldots, a_{n}\right)=(0, \ldots, 0)$, a contradiction to the fact that $\bar{x} \notin V$. Thus the theorem is proved when $d=0$.

We therefore assume the truth of the theorem for $\delta$-varieties of dimension less than $d$, and consider a $\delta$-variety $V$ of dimension $d$. Let $\mathbb{P}_{0}, \ldots, \mathbb{P}_{d}$ be $d+1$ independent generic $\delta$-hyperplanes passing through $\bar{x}$. The equation $\mathbb{P}_{d}$ can be written as $s_{1} y_{1}+\cdots+s_{n} y_{n}=0$ with $s_{i} \delta$-F-indeterminates. From Lemma 4.41, $\mathbb{P}_{d}=0$ meets $V$ in a $\delta$-variety $\mathbb{W}$ of dimension less than $d$. By the hypothesis of the induction, $\mathbb{P}_{0}, \ldots, \mathbb{P}_{d-1}$ do not meet $\mathbb{W}$; it follows that $V$ does not meet $\mathbb{P}_{0}, \ldots, \mathbb{P}_{d}$. Therefore the theorem is proved.

The following result proves the fourth statement of Theorem 1.2 .

Theorem 4.43. Let $F\left(\mathbf{u}_{0}, \mathbf{u}_{1}, \ldots, \mathbf{u}_{d}\right)$ be the Chow form of $V$ and $S_{F}=\frac{\partial F}{\partial u_{00}^{(h)}}$. Suppose that $\mathbf{u}_{i}(i=0, \ldots, d)$ are $\delta$-specialized over $\mathcal{F}$ to sets $\mathbf{v}_{i}$ of specific elements in $\mathcal{E}$ and $\overline{\mathbb{P}}_{i}(i=0, \ldots, d)$ are obtained by substituting $\mathbf{u}_{i}$ by $\mathbf{v}_{i}$ in $\mathbb{P}_{i}$. If $\overline{\mathbb{P}}_{i}=0(i=$ $0, \ldots, d)$ meet $V$, then $F\left(\mathbf{v}_{0}, \ldots, \mathbf{v}_{d}\right)=0$. Furthermore, if $F\left(\mathbf{v}_{0}, \ldots, \mathbf{v}_{d}\right)=0$ and $S_{F}\left(\mathbf{v}_{0}, \ldots, \mathbf{v}_{d}\right) \neq 0$, then the $d+1 \delta$-hyperplanes $\overline{\mathbb{P}}_{i}=0(i=0, \ldots, d)$ meet $V$.

Proof. Let $\mathcal{I}=\mathbb{I}(V) \subseteq \mathcal{F}\{\mathbb{Y}\}, \mathbb{I}_{\zeta, \xi}=\left[\mathcal{I}, \mathbb{P}_{0}, \ldots, \mathbb{P}_{d}\right] \subseteq \mathcal{F}\langle\mathbf{u}\rangle\left\{y_{1}, \ldots, y_{n}, u_{00}, \ldots, u_{d 0}\right\}$, and $\mathcal{I}_{1}=\left[\mathbb{I}_{\zeta, \xi}\right] \subseteq \mathcal{F}\left\langle\mathbf{u}_{0}, \ldots, \mathbf{u}_{d}\right\rangle\left\{y_{1}, \ldots, y_{n}\right\}$. By Lemma 4.10, $\left\{F, \frac{\partial F}{\partial u_{00}^{(h)}} y_{1}-\frac{\partial F}{\partial u_{01}^{(h)}}, \ldots\right.$, $\left.\frac{\partial F}{\partial u_{00}^{(h)}} y_{n}-\frac{\partial F}{\partial u_{0 n}^{(h)}}\right\}$ is a characteristic set of $\mathbb{I}_{\zeta, \xi}$ w.r.t. the elimination ranking $u_{d 0} \prec$ $\cdots \prec u_{00} \prec y_{1} \prec \cdots \prec y_{n}$. Since $F$ is irreducible, $\mathbb{I}_{\zeta, \xi}=\left[F, S_{F} y_{1}-\frac{\partial F}{\partial u_{01}^{(h)}}, \ldots, S_{F} y_{n}-\right.$ $\left.\frac{\partial F}{\partial u_{0 n}^{(h)}}\right]: S_{F}^{\infty}$ with $S_{F}=\frac{\partial F}{\partial u_{00}^{(h)}}$.

When $\mathbf{u}_{i}$ are $\delta$-specialized to $\mathbf{v}_{i}, \mathcal{I}_{1}$ becomes a $\delta$-ideal in $\mathcal{F}\left\langle\mathbf{v}_{0}, \ldots, \mathbf{v}_{d}\right\rangle\{\mathbb{Y}\}$. If $\overline{\mathbb{P}}_{0}, \ldots, \overline{\mathbb{P}}_{d}$ meet $V$, then $\overline{\mathcal{I}_{1}}=\left[\mathcal{I}, \overline{\mathbb{P}}_{0}, \ldots, \overline{\mathbb{P}}_{d}\right] \neq[1]$, which implies $F\left(\mathbf{v}_{0}, \ldots, \mathbf{v}_{d}\right)=0$ since $F \in \mathbb{I}_{\zeta, \xi}$.

If $S_{F}\left(\mathbf{v}_{0}, \ldots, \mathbf{v}_{d}\right) \neq 0$ and $F\left(\mathbf{v}_{0}, \ldots, \mathbf{v}_{d}\right)=0$, then let $\bar{y}_{i}=\left(\frac{\partial F}{\partial u_{0 i}^{(h)}}\left(\mathbf{v}_{0}, \ldots\right.\right.$, $\left.\left.\mathbf{v}_{d}\right)\right) /\left(S_{F}\left(\mathbf{v}_{0}, \ldots, \mathbf{v}_{d}\right)\right)(i=1, \ldots, n)$. We claim that $\left(\bar{y}_{1}, \ldots, \bar{y}_{n}\right)$ lies in $V$ and the $d+1 \delta$-hyperplanes $\overline{\mathbb{P}}_{0}, \ldots, \overline{\mathbb{P}}_{d}$, which implies that $\overline{\mathbb{P}}_{0}, \ldots, \overline{\mathbb{P}}_{d}$ meet $V$.

First, let $p$ be any $\delta$-polynomial in $\mathcal{I}$. Then $p \in \mathbb{I}_{\zeta, \xi}$, so there exists an integer $m$ such that $S_{F}^{m} p \in\left[F, S_{F} y_{1}-\frac{\partial F}{\partial u_{01}^{(h)}}, \ldots, S_{F} y_{n}-\frac{\partial F}{\partial u_{0 n}^{(h)}}\right]$. If we specialize $u_{i j} \rightarrow$ $v_{i j}, u_{i 0} \rightarrow v_{i 0}$ and let $y_{i}=\bar{y}_{i}$, then we have $S_{F}^{m}\left(\mathbf{v}_{0}, \ldots, \mathbf{v}_{d}\right) p\left(\bar{y}_{1}, \ldots, \bar{y}_{n}\right)=0$, so $p\left(\bar{y}_{1}, \ldots, \bar{y}_{n}\right)=0$. That is, $\left(\bar{y}_{1}, \ldots, \bar{y}_{n}\right) \in V$. Second, since $\mathbb{P}_{i} \in \mathbb{I}_{\zeta, \xi}$, similar to the above, it follows that $\left(\bar{y}_{1}, \ldots, \bar{y}_{n}\right)$ lies in $\overline{\mathbb{P}}_{i}$. So $\overline{\mathbb{P}}_{0}, \ldots, \overline{\mathbb{P}}_{d}$ meet $V$. 
Remark 4.44. Let $X$ be the set of all $(n-d-1)$-dimensional linear spaces in $\mathcal{F}^{n}$ that meet an irreducible $\delta$ - $\mathcal{F}$-variety $V$ of dimension $d$. From Theorem 4.43 , $X \subset \mathbb{V}(F)$ and $X \backslash \mathbb{V}\left(S_{F}\right)=\mathbb{V}(F) \backslash \mathbb{V}\left(S_{F}\right)$. That is, a "major" part of $X$ is known to be $\mathbb{V}(F) \backslash \mathbb{V}\left(S_{F}\right)$. An interesting problem is to see whether $X$ is a $\delta$-variety for a projective $\delta$-variety $V$. In 24, Kolchin showed that this problem has a positive answer in a special case; that is, $V$ is a projective algebraic variety which is treated as a zero-dimensional differential projective variety in a certain way.

Similar to the algebraic case [16, p.22], we can show that a generic $\delta$-hyperplane passing through a given point $x=\left(x_{1}, x_{2}, \ldots, x_{n}\right)$ is of the form $a_{0}+a_{1} y_{1}+\cdots+$ $a_{n} y_{n}=0$ with $a_{i}=\sum_{j=0}^{n} s_{i j} x_{j}(i=0,1, \ldots, n)$, where $x_{0}=1$ and $S=\left(s_{i j}\right)$ is an $(n+1) \times(n+1)$ skew-symmetric matrix with $s_{i j}(i<j)$ independent $\delta$ - $\mathcal{F}$ indeterminates in $\mathcal{E}$. That is,

$$
\left(\begin{array}{c}
a_{0} \\
a_{1} \\
\vdots \\
a_{n}
\end{array}\right)=S\left(\begin{array}{c}
1 \\
x_{1} \\
\vdots \\
x_{n}
\end{array}\right) .
$$

For convenience, we denote such a $\delta$-hyperplane by $S x$ and say a generic $\delta$-hyperplane passing through a point $x$ is of the form $S x$.

Now we write $\mathbf{u}_{i}=\left(u_{i 0}, u_{i 1}, \ldots, u_{i n}\right)^{T}=S^{i} Y$, where $Y=\left(1, y_{1}, \ldots, y_{n}\right)^{T}$ and the $S^{i}$ are skew-symmetric matrices with $s_{j k}^{i}(j<k)$ independent $\delta$ - $\mathcal{F}$-indeterminates in $\mathcal{E}$. Substituting the $\mathbf{u}_{i}$ in $F\left(\mathbf{u}_{0}, \mathbf{u}_{1}, \ldots, \mathbf{u}_{d}\right)$ by these equations, we obtain a $\delta$-polynomial involving $s_{j k}^{i}(j<k)$ and the $y_{l}$. Regarding this $\delta$-polynomial as a $\delta$ polynomial in $s_{j k}^{i}(j<k)$, then we have $F\left(\mathbf{u}_{0}, \mathbf{u}_{1}, \ldots, \mathbf{u}_{d}\right)=F\left(S^{0} Y, S^{1} Y, \ldots, S^{d} Y\right)$ $=\sum g_{\phi}\left(y_{1}, \ldots, y_{n}\right) \phi\left(s_{j k}^{i}\right)$, where $\phi\left(s_{j k}^{i}\right)$ are different $\delta$-monomials. In this way, we get a finite number of $\delta$-polynomials $g_{\phi}\left(y_{1}, \ldots, y_{n}\right)$ over $\mathcal{F}$, which is denoted by $\mathcal{P}$. Similarly, in this way, we will get another set $\mathcal{D}$ of $\delta$-polynomials from $S_{F}\left(\mathbf{u}_{0}, \ldots, \mathbf{u}_{d}\right)$.

Theorem 4.45. Let $V$ be an irreducible $\delta$ - $\mathcal{F}$-variety with dimension d and $F\left(\mathbf{u}_{0}, \mathbf{u}_{1}\right.$, $\left.\ldots, \mathbf{u}_{d}\right)$ its Chow form. Then $V \backslash \mathbb{V}(\mathcal{D})=\mathbb{V}(\mathcal{P}) \backslash \mathbb{V}(\mathcal{D}) \neq \emptyset$, where $\mathcal{P}, \mathcal{D}$ are the $\delta$ polynomial sets obtained from $F\left(\mathbf{u}_{0}, \mathbf{u}_{1}, \ldots, \mathbf{u}_{d}\right)$ and $S_{F}\left(\mathbf{u}_{0}, \mathbf{u}_{1}, \ldots, \mathbf{u}_{d}\right)$ respectively as above.

Proof. On the one hand, for any $\bar{x} \in V$, from Theorem 4.42, any $d+1$ generic $\delta$ hyperplanes passing through $\bar{x}$ meet $V$. So $S^{0} \bar{x}, S^{1} \bar{x}, \ldots, S^{d} \bar{x}$ meet $V$. By the proof of Theorem 4.43, $F\left(S^{0} \bar{x}, S^{1} \bar{x}, \ldots, S^{d} \bar{x}\right)=0$. Since $s_{j k}^{i}(j<k)$ are $\delta$-ndeterminates, $\bar{x} \in \mathbb{V}(\mathcal{P})$. So $V \backslash \mathbb{V}(\mathcal{D}) \subseteq \mathbb{V}(\mathcal{P}) \backslash \mathbb{V}(\mathcal{D})$.

On the other hand, for any $\bar{x} \in \mathbb{V}(\mathcal{P}) \backslash \mathbb{V}(\mathcal{D})$, since any $d+1$ generic $\delta$ hyperplanes passing through $\bar{x}$ are of the form $S^{0} \bar{x}, S^{1} \bar{x}, \ldots, S^{d} \bar{x}$ with the $S^{i} \delta$ indeterminate skew-symmetric matrices, we have $F\left(S^{0} \bar{x}, S^{1} \bar{x}, \ldots, S^{d} \bar{x}\right)=0$ and $S_{F}\left(S^{0} \bar{x}, S^{1} \bar{x}, \ldots, S^{d} \bar{x}\right) \neq 0$. From Theorem 4.43, $S^{0} \bar{x}, S^{1} \bar{x}, \ldots, S^{d} \bar{x}$ meet $V$. Thus from Theorem $4.42, \bar{x} \in V$. Thus $V \backslash \mathbb{V}(\mathcal{D})=\mathbb{V}(\mathcal{P}) \backslash \mathbb{V}(\mathcal{D})$.

Now, we show that $V \backslash \mathbb{V}(\mathcal{D}) \neq \emptyset$. Suppose the contrary, i.e. $V \subset \mathbb{V}(\mathcal{D})$, in particular, its generic point $\left(\xi_{1}, \ldots, \xi_{n}\right) \in \mathbb{V}(\mathcal{D})$. Thus, $S_{F}\left(S^{0} \xi, S^{1} \xi, \ldots, S^{d} \xi\right)=0$, where $\xi=\left(1, \xi_{1}, \ldots, \xi_{n}\right)$. Recall that $s_{j k}^{i}(j<k ; i=0,1, \ldots, d)$ are independent $\delta$-indeterminates over $\mathcal{F}\left\langle\xi_{1}, \ldots, \xi_{n}\right\rangle$. Now we consider a $\delta$-endomorphism $\phi$ of $\mathcal{F}\left\langle\xi_{1}, \ldots, \xi_{n}\right\rangle\left\{s_{j k}^{i}(j<k ; i=0,1, \ldots, d)\right\}$ over $\mathcal{F}\left\langle\xi_{1}, \ldots, \xi_{n}\right\rangle$ satisfying $\phi\left(s_{0 k}^{i}\right)=$ 
$-s_{0 k}^{i}$ and $\phi\left(s_{j k}^{i}\right)=0(j<k ; j=1, \ldots, n)$. It is clear that $\phi\left(S_{F}\left(S^{0} \xi, \ldots, S^{d} \xi\right)\right)=$ $S_{F}\left(s_{0 k}^{i} ;-\sum_{k=1}^{n} s_{0 k}^{0} \xi_{k}, \ldots,-\sum_{k=1}^{n} s_{0 k}^{d} \xi_{k}\right)=0$. Denoting $s_{0 k}^{i}$ by $u_{i k}$, we have $S_{F}(\mathbf{u}$; $\left.\zeta_{0}, \ldots, \zeta_{d}\right)=0$, thus $S_{F} \in \operatorname{sat}(F)$, which is a contradiction. So $V \backslash \mathbb{V}(\mathcal{D}) \neq \emptyset$.

Since $V$ is an irreducible $\delta$-variety, $V \cap \mathbb{V}(\mathcal{D})$ is a subset of $V$ with lower dimension than that of $V$ or with the same dimension but of lower order. Thus, $V \backslash \mathbb{V}(\mathcal{D})$ is an open set of $V$ in the Kolchin topology.

Example 4.46. Continue from Example 4.5. In this example, $F\left(\mathbf{u}_{0}\right)=u_{1}^{2}\left(u_{0}^{\prime}\right)^{2}-$ $2 u_{1} u_{1}^{\prime} u_{0} u_{0}^{\prime}+\left(u_{1}^{\prime}\right)^{2} u_{0}^{2}+4 u_{1}^{3} u_{0}$ and $S_{F}\left(\mathbf{u}_{0}\right)=2 u_{1}^{2} u_{0}^{\prime}-2 u_{1} u_{1}^{\prime} u_{0}$. Following the steps as above, we obtain $\mathcal{P}=\left\{\left(y_{1}^{\prime}\right)^{2}-4 y_{1}\right\}$ and $\mathcal{D}=\left\{y_{1}^{\prime}\right\}$. That is, we obtain the defining equation $\left(y_{1}^{\prime}\right)^{2}-4 y_{1}=0$ for the $\delta$-variety under the condition $y_{1}^{\prime} \neq 0$.

\section{Differential Chow variety}

In Theorem 1.2, we have listed four properties for the differential Chow form. In this section, we are going to prove that these properties are also the sufficient conditions for a $\delta$-polynomial $F\left(\mathbf{u}_{0}, \ldots, \mathbf{u}_{d}\right)$ to be the Chow form for a $\delta$-variety. Based on these sufficient conditions, we can define the $\delta$-Chow quasi-variety for certain classes of $\delta$-varieties in the sense that a point in the $\delta$-Chow quasi-variety represents a $\delta$-variety in the class. In other words, we give a parametrization of all $\delta$-varieties in the class. Obviously, this is an extension of the concept of Chow variety in the algebraic case [13, 16.

5.1. Sufficient conditions for a polynomial to be a differential Chow form. The following result gives sufficient conditions for a $\delta$-polynomial to be the Chow form of an irreducible $\delta$-variety. From Theorem 1.2, they are also necessary conditions.

Theorem 5.1. Let $F\left(\mathbf{u}_{0}, \mathbf{u}_{1}, \ldots, \mathbf{u}_{d}\right)$ be an irreducible $\delta$-polynomial in $\mathcal{F}\left\{\mathbf{u}_{0}, \mathbf{u}_{1}\right.$, $\left.\ldots, \mathbf{u}_{d}\right\}$, where $\mathbf{u}_{i}=\left(u_{i 0}, u_{i 1}, \ldots, u_{i n}\right)(i=0, \ldots, d)$. If $F$ satisfies the following conditions, then it is the Chow form for an irreducible $\delta$-variety of dimension $d$ and order $h$.

1. $F\left(\mathbf{u}_{0}, \mathbf{u}_{1}, \ldots, \mathbf{u}_{d}\right)$ is $\delta$-homogenous of the same degree in each set of $\delta$-variables $\mathbf{u}_{i}$ and $\operatorname{ord}\left(F, u_{i j}\right)=h$ for all $u_{i j}$ occurring in $F$.

2. $F\left(\mathbf{u}_{0}, \mathbf{u}_{1}, \ldots, \mathbf{u}_{d}\right)$ can be factored uniquely into the following form:

$$
\begin{aligned}
F\left(\mathbf{u}_{0}, \mathbf{u}_{1}, \ldots, \mathbf{u}_{d}\right) & =A\left(\mathbf{u}_{0}, \mathbf{u}_{1}, \ldots, \mathbf{u}_{d}\right) \prod_{\tau=1}^{g}\left(u_{00}^{(h)}+\sum_{\rho=1}^{n} u_{0 \rho}^{(h)} \xi_{\tau \rho}+t_{\tau}\right) \\
& =A\left(\mathbf{u}_{0}, \mathbf{u}_{1}, \ldots, \mathbf{u}_{d}\right) \prod_{\tau=1}^{g}\left(u_{00}+\sum_{\rho=1}^{n} u_{0 \rho} \xi_{\tau \rho}\right)^{(h)},
\end{aligned}
$$

where $g=\operatorname{deg}\left(F, u_{00}^{(h)}\right)$ and $\xi_{\tau \rho}$ are in a differential extension field $\mathcal{F}_{\tau}$ of $\mathcal{F}$. The first " =" is obtained by factoring $F\left(\mathbf{u}_{0}, \mathbf{u}_{1}, \ldots, \mathbf{u}_{d}\right)$ as an algebraic polynomial in the variables $u_{00}^{(h)}, u_{01}^{(h)}, \ldots, u_{0 n}^{(h)}$, while the second one is a differential expression by defining the derivatives of $\xi_{\tau \rho}$ to be

$$
\xi_{\tau \rho}^{(m)}=\left.\left(\delta \xi_{\tau \rho}^{(m-1)}\right)\right|_{u_{00}^{(h)}=-\sum_{\rho=1}^{n} u_{0 \rho}^{(h)} \xi_{\tau \rho}-t_{\tau}}(m \geq 1)
$$

recursively. 
3. $\Xi_{\tau}=\left(\xi_{\tau 1}, \ldots, \xi_{\tau n}\right)(\tau=1, \ldots, g)$ are on the $\delta$-hyperplanes $\mathbb{P}_{\sigma}=0(\sigma=$ $1, \ldots, d)$ as well as on the algebraic hyperplanes ${ }^{a} \mathbb{P}_{0}^{(k)}=0(k=0, \ldots, h-1)$.

4. For each $\tau$, if $v_{i 0}+v_{i 1} \xi_{\tau 1}+\cdots+v_{i n} \xi_{\tau n}=0(i=0, \ldots, d)$, then $F\left(\mathbf{v}_{0}, \ldots, \mathbf{v}_{d}\right)=$ 0 , where $\mathbf{v}_{i}=\left(v_{i 0}, v_{i 1}, \ldots, v_{i n}\right)$ and $v_{i j} \in \mathcal{E}$. Equivalently, if $S^{0}, \ldots, S^{d}$ are $(n+1) \times$ $(n+1)$ skew-symmetric matrices, each having independent $\delta$-indeterminates above its principle diagonal, then $F\left(S^{0} \xi_{\tau}, \ldots, S^{d} \xi_{\tau}\right)=0$, where $\xi_{\tau}=\left(1, \xi_{\tau 1}, \ldots, \xi_{\tau n}\right)$.

Before proving the theorem, we need several lemmas.

Lemma 5.2 ([15, p.11, Theorem 1]). Let $\mathcal{R}$ and $\mathcal{S}^{*}$ be two rings and $\mathcal{R}$ isomorphic to a subring $\mathcal{S}$ of $\mathcal{S}^{*}$. Then there exists an extension ring $\mathcal{R}^{*}$ of $\mathcal{R}$ such that this isomorphism can be extended to an isomorphism between $\mathcal{R}^{*}$ and $\mathcal{S}^{*}$.

Lemma 5.3. Let $V$ be an irreducible $\delta$-F-variety of dimension $d>0$ and $\mathbb{P}=$ $u_{0}+u_{1} y_{1}+\cdots+u_{n} y_{n}$ be a generic $\delta$-hyperplane where $u_{i} \in \mathcal{E}$. Then every generic point of $\mathbb{V}(\mathbb{I}(V), \mathbb{P})$ over $\mathcal{F}_{1}=\mathcal{F}\left\langle u_{0}, \ldots, u_{n}\right\rangle$ is a generic point of $V$ over $\mathcal{F}$.

Proof. By Theorem 3.7, $[\mathbb{I}(V), \mathbb{P}]$ is a prime $\delta$-ideal of dimension $d-1$ in $\mathcal{F}_{1}\{\mathbb{Y}\}$. Let $\eta$ be a generic point of $\mathbb{V}(\mathbb{I}(V), \mathbb{P})$. Then for any $\delta$-polynomial $p$ in $\mathbb{I}(V)$, we have $p(\eta)=0$. On the other hand, for any $\delta$-polynomial $p \in \mathcal{F}\left\{y_{1}, \ldots, y_{n}\right\}$ such that $p(\eta)=0$, we have $p \in[\mathbb{I}(V), \mathbb{P}]$. Then $p \equiv \sum_{i} h_{i} \mathbb{P}^{(i)} \bmod \mathbb{I}(V)$. Substituting $u_{0}$ by $-u_{1} y_{1}-\cdots-u_{n} y_{n}$ in the above equality, we have $p \equiv 0 \bmod \mathbb{I}(V)$. Hence $\eta$ is a generic point of $V$.

In the next result, we will show that if condition 4 from Theorem 5.1 holds, then the following stronger version is also valid.

Lemma 5.4. Let $F$ satisfy condition 4 of Theorem 5.1. Consider $F$ as an algebraic polynomial $f\left(u_{\sigma j}^{(k)}, u_{0 j}^{(l)}, u_{00}^{(h)}, \ldots, u_{0 n}^{(h)}\right)$ in $u_{i j}^{(k)}$, and $\left(\xi_{\tau 1}, \ldots, \xi_{\tau n}, \ldots, \xi_{\tau 1}^{(h)}, \ldots, \xi_{\tau n}^{(h)}\right)$ is regarded as an algebraic point. If $w_{i 0 k}+\sum_{j=1}^{n} \sum_{m=0}^{k}\left(\begin{array}{c}k \\ m\end{array}\right) w_{i j m} \delta_{\tau}^{(k-m)} \xi_{\tau j}=0(i=$ $0, \ldots, d ; k=0, \ldots, h)$, then $f\left(w_{\sigma j k}, w_{0 j l}, w_{00 h}, \ldots, w_{0 n h}\right)=0$, where the $w_{i j k}$ are considered as elements in the underlying ordinary field of $\mathcal{E}$.

Proof. Regard $\mathcal{Q}_{\tau}=\left[v_{i 0}+\sum_{j=1}^{n} v_{i j} \xi_{\tau j}: i=0, \ldots, d\right]$ as a $\delta$-ideal in $\mathcal{F}\left\langle\xi_{\tau j}\right.$ : $j=1, \ldots, n\rangle\left\{v_{i 0}, \ldots, v_{i n}: i=0, \ldots, d\right\}$, where $v_{i j}$ are $\delta$ - $\mathcal{F}\left\langle\xi_{\tau j}\right\rangle$-indeterminates. From condition 4 of Theorem [5.1, $\left.F\left(\mathbf{v}_{0}, \ldots, \mathbf{v}_{d}\right)\right|_{\mathbb{V}\left(\mathcal{Q}_{\tau}\right)} \equiv 0$. It is clear that $\mathcal{Q}_{\tau}$ is a prime $\delta$-ideal and $\left\{v_{i 0}+\sum_{j=1}^{n} v_{i j} \xi_{\tau j}: i=0, \ldots, d\right\}$ is its characteristic set with $v_{i 0}$ as leaders. By the differential Nullstellensatz, $F\left(\mathbf{v}_{0}, \ldots, \mathbf{v}_{d}\right) \in \mathcal{Q}_{\tau}$. From condition 1 of Theorem [5.1 $\operatorname{ord}\left(F, v_{i 0}\right)=h$. Then $F\left(\mathbf{v}_{0}, \ldots, \mathbf{v}_{d}\right) \in\left(v_{i 0}+\right.$ $\left.\sum_{j=1}^{n} v_{i j} \xi_{\tau j}, \ldots, v_{i 0}^{(h)}+\sum_{j=1}^{n} v_{i j}^{(h)} \xi_{\tau j}+\sum_{j=1}^{n} \sum_{m=0}^{h-1}\left(\begin{array}{c}h \\ m\end{array}\right) v_{i j}^{(m)} \xi_{\tau j}^{(h-m)}: i=0, \ldots, d\right)$. Regarding the above relation as a pure algebraic relation, we can substitute $v_{i j}^{(k)}$ by algebraic indeterminates $w_{i j k}$ and regard $F$ as an algebraic polynomial. Then $f\left(w_{\sigma j k}, w_{0 j l}, w_{00 h}, \ldots, w_{0 n h}\right) \in\left(w_{i 00}+\sum_{j=1}^{n} w_{i j 0} \xi_{\tau j}, \ldots, w_{i 0 h}+\sum_{j=1}^{n} w_{i j h} \xi_{\tau j}+\right.$ $\left.\sum_{j=1}^{n} \sum_{m=0}^{h-1}\left(\begin{array}{c}h \\ m\end{array}\right) w_{i j m} \xi_{\tau j}^{(h-m)}: i=0, \ldots, d\right)$, which shows that the lemma is valid.

Proof of Theorem 5.1. Let $V_{\tau}(\tau=1, \ldots, g)$ be the irreducible $\delta$ - $\mathcal{F}$-variety with $\left(\xi_{\tau_{1}}, \ldots, \xi_{\tau n}\right)$ as its generic point over $\mathcal{F}$. We will later show that all the $\delta$-varieties $V_{\tau}$ are the same.

First, we claim that the generic points of $V_{\tau}$ which lie on $\mathbb{P}_{1}, \ldots, \mathbb{P}_{d}$ as well as on ${ }^{a} \mathbb{P}_{0},{ }^{a} \mathbb{P}_{0}^{\prime}, \ldots,{ }^{a} \mathbb{P}_{0}^{(h-1)}$ are included in $\left\{\left(\xi_{\tau 1}, \ldots, \xi_{\tau n}\right): \tau=1, \ldots, g\right\}$. Without 
loss of generality, we consider $V_{1}$. To prove that the claim holds for $V_{1}$, we need to use the following assertion:

$(\star)$ If $\left(\eta_{10}, \ldots, \eta_{n 0}, \ldots, \eta_{1 h}, \ldots, \eta_{n h}\right)$ is a generic point of the algebraic ideal $\mathbb{I}\left(V_{1}\right)^{\langle h\rangle}=\mathbb{I}\left(V_{1}\right) \cap \mathcal{F}\left[y_{1}, \ldots, y_{n}, \ldots, y_{1}^{(h)}, \ldots, y_{n}^{(h)}\right]$ which lies on ${ }^{a} \mathbb{P}_{\sigma}^{(k)},{ }^{a} \mathbb{P}_{0}^{(l)}(\sigma=$ $1, \ldots, d ; k=0, \ldots, h ; l=0, \ldots, h-1)$, then there must exist some $\tau$ such that $\eta_{j 0}=\xi_{\tau j}$ for $j=1, \ldots, n$.

Assume that $(\star)$ is valid. Suppose $\eta=\left(\eta_{1}, \ldots, \eta_{n}\right) \in \mathcal{E}^{n}$ is a generic point of $V_{1}$ which lie on $\mathbb{P}_{1}, \ldots, \mathbb{P}_{d}$ as well as on ${ }^{a} \mathbb{P}_{0},{ }^{a} \mathbb{P}_{0}^{\prime}, \ldots,{ }^{a} \mathbb{P}_{0}^{(h-1)}$. Then the algebraic point $\left(\eta_{1}, \ldots, \eta_{n}, \ldots, \eta_{1}^{(h)}, \ldots, \eta_{n}^{(h)}\right)$ is a generic point of the algebraic ideal $\mathbb{I}\left(V_{1}\right)^{\langle h\rangle}=$ $\mathbb{I}\left(V_{1}\right) \cap \mathcal{F}\left[y_{1}, \ldots, y_{n}, \ldots, y_{1}^{(h)}, \ldots, y_{n}^{(h)}\right]$ which lies on ${ }^{a} \mathbb{P}_{\sigma}^{(k)}, a \mathbb{P}_{0}^{(l)}(\sigma=1, \ldots, d ; k=$ $0, \ldots, h ; l=0, \ldots, h-1)$. By $(\star)$, there must exist some $\tau$ such that $\eta_{j}=\xi_{\tau j}$ for $j=1, \ldots, n$. Since $\eta$ is a differential point of $V_{1}$, thus $\eta=\xi_{\tau}$ for some $\tau$.

Now we are going to prove $(\star)$. Similar to the proof of Lemma 5.4, rewrite $F$ as an algebraic polynomial $f\left(u_{\sigma j}^{(k)}, u_{0 j}^{(l)}, u_{00}^{(h)}, \ldots, u_{0 n}^{(h)}\right)$ and consider condition 2 as a pure algebraic factorization. Let $\left(\eta_{10}, \ldots, \eta_{n 0}, \ldots, \eta_{1 h}, \ldots, \eta_{n h}\right)$ be such a generic point of $\mathbb{I}\left(V_{1}\right)^{\langle h\rangle}$ other than $\left(\xi_{11}, \ldots, \xi_{1 n}, \ldots, \xi_{11}^{(h)}, \ldots, \xi_{1 n}^{(h)}\right)$. Then we have the $\mathcal{F}$ isomorphism $\mathcal{F}\left(\eta_{10}, \ldots, \eta_{n 0}, \ldots, \eta_{1 h}, \ldots, \eta_{n h}\right) \cong \mathcal{F}\left(\xi_{11}, \ldots, \xi_{1 n}, \ldots, \xi_{11}^{(h)}, \ldots, \xi_{1 n}^{(h)}\right)$, which maps $\eta_{j k}$ to $\xi_{1 j}^{(k)}$ for $j=1, \ldots, n$ and $k=0, \ldots, h$. By Lemma [5.2, there exist $w_{\sigma j k}, w_{0 j l} \in \mathcal{E}$ such that the above algebraic isomorphism can be extended to the isomorphism

$$
\mathcal{F}\left(\eta_{10}, \ldots, \eta_{n 0}, \ldots, \eta_{1 h}, \ldots, \eta_{n h}, u_{\sigma j}^{(k)}, u_{0 j}^{(l)}\right) \cong \mathcal{F}\left(\xi_{11}, \ldots, \xi_{1 n}, \ldots, \xi_{11}^{(h)}, \ldots, \xi_{1 n}^{(h)}, w_{\sigma j k}, w_{0 j l}\right)
$$

where $\sigma=1, \ldots, d ; j=0, \ldots, n ; k=0, \ldots, h ; l=0, \ldots, h-1$, and $u_{\sigma j}^{(k)}$ and $u_{0 j}^{(l)}$ map to $w_{\sigma j k}$ and $w_{0 j l}$, respectively. Since $\left(\eta_{10}, \ldots, \eta_{n 0}, \ldots, \eta_{1 h}, \ldots, \eta_{n h}\right)$ lies on ${ }^{a} \mathbb{P}_{\sigma}^{(k)},{ }^{a} \mathbb{P}_{0}^{(l)}$, the relation $u_{\sigma 0}^{(k)}+\sum_{j=1}^{n} \sum_{m=0}^{k}\left(\begin{array}{c}k \\ m\end{array}\right) u_{\sigma j}^{(m)} \eta_{j, k-m}=0$ implies that $w_{\sigma 0 k}+\sum_{j=1}^{n} \sum_{m=0}^{k}\left(\begin{array}{c}k \\ m\end{array}\right) w_{\sigma j m} \xi_{1 j}^{(k-m)}=0(\sigma=1, \ldots, d)$ and the relation $u_{00}^{(l)}+$ $\sum_{j=1}^{n} \sum_{m=0}^{l}\left(\begin{array}{c}l \\ m\end{array}\right) u_{0 j}^{(m)} \eta_{j, l-m}=0$ implies that $w_{00 l}+\sum_{j=1}^{n} \sum_{m=0}^{l}\left(\begin{array}{c}l \\ m\end{array}\right) w_{0 j m} \xi_{1 j}^{(l-m)}$ $=0(l=0, \ldots, h-1)$. Furthermore, if $w_{00 h}+\sum_{i=1}^{n} w_{0 i h} \xi_{1 i}^{(h)}+\sum_{j=1}^{n} \sum_{m=0}^{h-1}\left(\begin{array}{c}h \\ m\end{array}\right)$ $w_{0 j m} \xi_{1 j}^{(h-m)}=0$ is valid, then from Lemma 5.4 it follows that the algebraic polynomial $f\left(w_{\sigma j k}, w_{0 j l}, w_{00 h}, \ldots, w_{0 n h}\right)=0$. Then, by the Hilbert Nullstellensatz, when regarded as a polynomial in the algebraic indeterminates $u_{00}^{(h)}, \ldots, u_{0 n}^{(h)}$, $f\left(w_{\sigma j k}, w_{0 j l}, u_{00}^{(h)}, \ldots, u_{0 n}^{(h)}\right) \in\left(u_{00}^{(h)}+u_{01}^{(h)} \xi_{11}+\cdots+u_{0 n}^{(h)} \xi_{1 n}+\sum_{j=1}^{n} \sum_{m=0}^{h-1}\left(\begin{array}{c}h \\ m\end{array}\right)\right.$ $\left.w_{0 j m} \xi_{1 j}^{(h-m)}\right)$. Thus, $u_{00}^{(h)}+u_{01}^{(h)} \xi_{11}+\cdots+u_{0 n}^{(h)} \xi_{1 n}+\sum_{j=1}^{n} \sum_{m=0}^{h-1}\left(\begin{array}{c}h \\ m\end{array}\right) w_{0 j m} \xi_{1 j}^{(h-m)}$ divides $f\left(w_{\sigma j k}, w_{0 j l}, u_{00}^{(h)}, \ldots, u_{0 n}^{(h)}\right)$. By the above isomorphism, $f\left(u_{\sigma j}^{(k)}, u_{0 j}^{(l)}, u_{00}^{(h)}\right.$, $\left.\ldots, u_{0 n}^{(h)}\right)$ is divisible by $u_{00}^{(h)}+u_{01}^{(h)} \eta_{10}+\cdots+u_{0 n}^{(h)} \eta_{n 0}+\sum_{j=1}^{n} \sum_{m=0}^{h-1}\left(\begin{array}{c}h \\ m\end{array}\right) u_{0 j}^{(m)} \eta_{j, h-m}$. The first factorization expression of condition 2 shows that when regarded as an algebraic polynomial in the variables $u_{00}^{(h)}, u_{01}^{(h)}, \ldots, u_{0 n}^{(h)}, f\left(u_{\sigma j}^{(k)}, u_{0 j}^{(l)}, u_{00}^{(h)}, \ldots, u_{0 n}^{(h)}\right)$ $=A \prod_{\tau=1}^{g}\left(u_{00}^{(h)}+u_{01}^{(h)} \xi_{\tau 1}+\cdots+u_{0 n}^{(h)} \xi_{\tau n}+\sum_{j=1}^{n} \sum_{k=0}^{h-1}\left(\begin{array}{c}h \\ k\end{array}\right) u_{0 j}^{(k)} \xi_{\tau j}^{(h-k)}\right)$. Thus, there exist some $\tau$ such that $\eta_{j 0}=\xi_{\tau j}(j=1, \ldots, n)$, which completes the proof of the claim.

Denote the dimension and order of $V_{\tau}$ by $d_{\tau}$ and $h_{\tau}$, respectively. We claim that $d_{\tau}=d$ and $h_{\tau}=h$. Since $V_{\tau}$ meets $\mathbb{P}_{1}, \ldots, \mathbb{P}_{d}$ and $\left(\xi_{\tau 1}, \ldots, \xi_{\tau n}\right)$ are such points in their intersection variety, by Theorem $3.7, d_{\tau} \geq d$. If $d_{\tau}>d \geq 0$, then $V_{\tau}$ meets 
$\mathbb{P}_{1}, \ldots, \mathbb{P}_{d}, \mathbb{P}_{0}$. Let $\left(\eta_{1}, \ldots, \eta_{n}\right)$ be a generic point of $\mathbb{V}\left(\mathbb{I}\left(V_{\tau}\right), \mathbb{P}_{1}, \ldots, \mathbb{P}_{d}, \mathbb{P}_{0}\right)$. Then by Lemma 5.3, $\left(\eta_{1}, \ldots, \eta_{n}\right)$ is also a generic point of $V_{\tau}$. Since $\left(\eta_{1}, \ldots, \eta_{n}\right)$ lies on $\mathbb{P}_{0}$, it also lies on ${ }^{a} \mathbb{P}_{0},{ }^{a} \mathbb{P}_{0}^{\prime}, \ldots,{ }^{a} \mathbb{P}_{0}^{(h-1)}$. From the above claim, there exist some $\tau$ such that $\left(\eta_{1}, \ldots, \eta_{n}\right)=\left(\xi_{\tau 1}, \ldots, \xi_{\tau n}\right)$. Thus, $\left(\xi_{\tau 1}, \ldots, \xi_{\tau n}\right)$ lies on $\mathbb{P}_{0}$, which implies that $F\left(\mathbf{u}_{0}, \ldots, \mathbf{u}_{d}\right)$ is a zero $\delta$-polynomial, which is a contradiction. So $d_{\tau}=d$.

It remains to show that $h_{\tau}=h$. We first prove $h_{\tau} \geq h$. Suppose the contrary; then $h_{\tau} \leq h-1$. Similar to the proof of Theorem 4.36, we can prove that $\mathbb{V}\left(\left[\mathbb{I}\left(V_{\tau}\right), \mathbb{P}_{1}, \ldots, \mathbb{P}_{d}\right]^{\langle h\rangle},{ }^{a} \mathbb{P}_{0}, \ldots,{ }^{a} \mathbb{P}_{0}^{(h-1)}\right)=\emptyset$. But $\left(\xi_{\tau_{1}}, \ldots, \xi_{\tau n}\right)$ is an element of $\mathbb{V}\left(\mathbb{I}\left(V_{\tau}\right), \mathbb{P}_{1}, \ldots, \mathbb{P}_{d}\right)$ which also lies on ${ }^{a} \mathbb{P}_{0},{ }^{a} \mathbb{P}_{0}^{\prime}, \ldots,{ }^{a} \mathbb{P}_{0}^{(h-1)}$, which is a contradiction. Now suppose that $h_{\tau}>h$; then $h_{\tau}-1 \geq h$. From Theorems 4.36 and 4.34, every point of $V_{\tau}$ which lies both on $\mathbb{P}_{1}, \ldots, \mathbb{P}_{d}$ and on ${ }^{a} \mathbb{P}_{0},{ }^{a} \mathbb{P}_{0}^{\prime}, \ldots,{ }^{a} \mathbb{P}_{0}^{\left(h_{\tau}-1\right)}$ is a generic point of $V_{\tau}$. But the generic points of $V_{\tau}$ which lie on $\mathbb{P}_{1}, \ldots, \mathbb{P}_{d}$ as well as on ${ }^{a} \mathbb{P}_{0},{ }^{a} \mathbb{P}_{0}^{\prime}, \ldots,{ }^{a} \mathbb{P}_{0}^{(h-1)}$ are included in $\left\{\left(\xi_{\tau 1}, \ldots, \xi_{\tau n}\right): \tau=1, \ldots, g\right\}$. So some $\left(\xi_{\tau 1}, \ldots, \xi_{\tau n}\right)$ lies on ${ }^{a} \mathbb{P}_{0},{ }^{a} \mathbb{P}_{0}^{\prime}, \ldots,{ }^{a} \mathbb{P}_{0}^{\left(h_{\tau}-1\right)}$. Since $h_{\tau}-1 \geq h$, we have that $\left(\xi_{\tau 1}, \ldots, \xi_{\tau n}\right)$ lies on ${ }^{a} \mathbb{P}_{0}^{(h)}$, which implies $F\left(\mathbf{u}_{0}, \ldots, \mathbf{u}_{d}\right) \equiv 0$, a contradiction. Thus, we have proved that $d_{\tau}=d$ and $h_{\tau}=h$.

Since the solutions of $V_{\tau}$ and $\mathbb{P}_{1}, \ldots, \mathbb{P}_{d}$ which also lie on ${ }^{a} \mathbb{P}_{0},{ }^{a} \mathbb{P}_{0}^{\prime}, \ldots,{ }^{a} \mathbb{P}_{0}^{(h-1)}$ are generic points of $V_{\tau}$, these are therefore contained in $\left\{\left(\xi_{\tau 1}, \ldots, \xi_{\tau n}\right): \tau=1, \ldots, g\right\}$. Hence, the differential Chow form of $V_{\tau}$ is of the form

$$
F_{\tau}\left(\mathbf{u}_{0}, \ldots, \mathbf{u}_{d}\right)=A_{\tau} \prod_{\rho=1}^{g}\left(\left(u_{00}+u_{01} \xi_{\tau 1}+\cdots+u_{0 n} \xi_{\tau n}\right)^{(h)}\right)^{l_{\tau \rho}},
$$

where $l_{\tau \rho}=1$ or 0 according to whether $\left(\xi_{\tau 1}, \ldots, \xi_{\tau n}\right)$ is in $V_{\tau}$. Since both $F_{\tau}$ and $F$ are irreducible, they differ at most by a factor in $\mathcal{F}$. Therefore, $V_{\tau}(\tau=1, \ldots, g)$ are the same $\delta$-variety, and $F\left(\mathbf{u}_{0}, \ldots, \mathbf{u}_{d}\right)$ is their differential Chow form.

In order to define $\delta$-Chow varieties in the next subsection, we will introduce the concept of order-unmixed $\delta$-varieties. A $\delta$-variety $V$ is called order-unmixed if all its components have the same dimension and order. Let $V$ be an order-unmixed $\delta$-variety with dimension $d$ and order $h$ and $V=\bigcup_{i=1}^{l} V_{i}$ its minimal irreducible decomposition with $F_{i}\left(\mathbf{u}_{0}, \mathbf{u}_{1}, \ldots, \mathbf{u}_{d}\right)$ the Chow form of $V_{i}$. Let

$$
F\left(\mathbf{u}_{0}, \ldots, \mathbf{u}_{d}\right)=\prod_{i=1}^{l} F_{i}\left(\mathbf{u}_{0}, \mathbf{u}_{1}, \ldots, \mathbf{u}_{d}\right)^{s_{i}}
$$

with $s_{i}$ arbitrary nonnegative integers. Associated with (5.1), we introduce the concept of a differential algebraic cycle, or simply a $\delta$-cycle, with $\mathbf{V}=\sum_{i=1}^{l} s_{i} \mathbf{V}_{i}$ as a generalization of the concept of an algebraic cycle in algebraic geometry [13, where $s_{i}$ is called the multiplicity of $\mathbf{V}_{i}$ in $\mathbf{V}$. Recall that we have defined the $\delta$-degree $m$ and leading $\delta$-degree $g$ for an irreducible $\delta$-variety $V$ in Definitions 4.19 and 4.33. respectively. Let $g_{i}$ and $m_{i}$ be the leading $\delta$-degree and $\delta$-degree of $V_{i}$ respectively. Then the leading $\delta$-degree and $\delta$-degree of $\mathbf{V}$ is defined to be $\sum_{i=1}^{l} s_{i} g_{i}$ and $\sum_{i=1}^{l} s_{i} m_{i}$, respectively.

Given a $\delta$-polynomial $G\left(\mathbf{u}_{0}, \ldots, \mathbf{u}_{d}\right)$ with $\operatorname{ord}\left(G, u_{00}\right)=h$, it may be reducible over $\mathcal{F}$ such that some of its irreducible factors are free of $u_{00}^{(h)}$. In that case, if the product of all such factors is $L$, then we define the primitive part of $G$ w.r.t. $u_{00}^{(h)}$ 
to be $G / L$. Otherwise, its primitive part w.r.t. $u_{00}^{(h)}$ by convention is defined to be itself. Then we have

Theorem 5.5. Let $F\left(\mathbf{u}_{0}, \mathbf{u}_{1}, \ldots, \mathbf{u}_{d}\right)$ be a $\delta$-polynomial and $\widetilde{F}$ the primitive part of $F$ with respect to the variable $u_{00}^{(h)}$. If $F$ satisfies the four conditions in Theorem 5.1, then $\widetilde{F}$ is the Chow form for the $\delta$-cycle of dimension $d$ and order $h$.

Proof. By definition, $F=B \widetilde{F}$, where $\operatorname{ord}\left(B, \mathbf{u}_{0}\right)<h$. Since $F$ is $\delta$-homogenous in $\mathbf{u}_{i}$ for each $i, \widetilde{F}$ is $\delta$-homogenous in each $\mathbf{u}_{i}$, too. Also, since $B$ is free of $u_{00}^{(h)}$, i.e. $B$ divides $A$, then $\widetilde{F}$ satisfies conditions 2 and 3 , and moreover the $\left(\xi_{\tau 1}, \ldots, \xi_{\tau n}\right)$ in the factorization are the same as that of $F$. By the proof of Theorem 5.1, we have that $\mathbb{I}\left(\xi_{\tau 1}, \ldots, \xi_{\tau n}\right)$ is of dimension $d$ and order $h$ over $\mathcal{F}$. Then similar to the proof of Lemma 4.1 and Theorem 4.11, we conclude that $\mathbb{I}\left(-\sum_{j=1}^{n} v_{0 j} \xi_{\tau j}, \ldots,-\sum_{j=1}^{n} v_{d j} \xi_{\tau j}\right)$ is of dimension $d$ over $\mathcal{F}\left\langle v_{i j}: i=0, \ldots, d ; j=\right.$ $1, \ldots, n\rangle$ and its relative order w.r.t. any parametric set is $h$, where $v_{i j}(i=$ $0, \ldots, d ; j=1, \ldots, n)$ are $\delta$-indeterminates over $\mathcal{F}\left\langle\xi_{\tau 1}, \ldots, \xi_{\tau n}\right\rangle$. In particular, $\operatorname{tr} . \operatorname{deg} \mathcal{F}\left\langle\zeta_{0}, \zeta_{1}, \ldots, \zeta_{d}\right\rangle / \mathcal{F}\left\langle\zeta_{1}, \ldots, \zeta_{d}\right\rangle=h$, where $\zeta_{i}=-\sum_{j=1}^{n} v_{i j} \xi_{\tau j}$. Thus $B\left(-\sum_{j=1}^{n} v_{0 j} \xi_{\tau j}, \ldots,-\sum_{j=1}^{n} v_{d j} \xi_{\tau j}\right) \neq 0$. But $F\left(-\sum_{j=1}^{n} v_{0 j} \xi_{\tau j}, \ldots,-\sum_{j=1}^{n} v_{d j} \xi_{\tau j}\right)$ $=0$, so $\widetilde{F}\left(-\sum_{j=1}^{n} v_{0 j} \xi_{\tau j}, \ldots,-\sum_{j=1}^{n} v_{d j} \xi_{\tau j}\right)=0$. It follows that $\widetilde{F}\left(S^{0} \xi_{\tau}, \ldots, S^{d} \xi_{\tau}\right)$ $=0$, for if we suppose the contrary, then $B\left(S^{0} \xi_{\tau}, \ldots, S^{d} \xi_{\tau}\right)=0$. But if we specialize $s_{j k}^{i}(j<k, j>0)$ to 0 and $s_{0 k}^{i}(k>0)$ to $-v_{i k}$, then

$$
B\left(-\sum_{j=1}^{n} v_{0 j} \xi_{\tau j}, \ldots,-\sum_{j=1}^{n} v_{d j} \xi_{\tau j}\right)=0,
$$

which is a contradiction. Thus, $\widetilde{F}$ satisfies condition 4 .

Now we claim that $\widetilde{F}$ is the Chow form of some $\delta$-cycle. Let $V_{\tau}(\tau=1, \ldots, g)$ be the irreducible $\delta$ - $\mathcal{F}$-variety with $\left(\xi_{\tau_{1}}, \ldots, \xi_{\tau n}\right)$ as its generic point over $\mathcal{F}$. Following the steps in the proof of Theorem 5.1 exactly, we arrive at the conclusion that the Chow form of $V_{\tau}$ is of the form

$$
F_{\tau}\left(\mathbf{u}_{0}, \ldots, \mathbf{u}_{d}\right)=A_{\tau} \prod_{\rho=1}^{g}\left(\left(u_{00}+u_{01} \xi_{\tau 1}+\cdots+u_{0 n} \xi_{\tau n}\right)^{(h)}\right)^{l_{\tau \rho}},
$$

where $l_{\tau \rho}=1$ or 0 according to whether $\left(\xi_{\tau 1}, \ldots, \xi_{\tau n}\right)$ is in $V_{\tau}$. Since each $\xi_{\tau}$ is in at least one of the $\delta$-varieties $V_{i}$, the Chow form of $\bigcup_{\tau=1}^{g} V_{\tau}$ is of the form $G\left(\mathbf{u}_{0}, \ldots, \mathbf{u}_{d}\right)=\prod_{\tau=1}^{g}\left(F_{\tau}\left(\mathbf{u}_{0}, \ldots, \mathbf{u}_{d}\right)\right)^{s_{\tau}}=C\left(\mathbf{u}_{0}, \ldots, \mathbf{u}_{d}\right) \prod_{\rho=1}^{g}\left(\left(u_{00}+u_{01} \xi_{\tau 1}+\right.\right.$ $\left.\left.\cdots+u_{0 n} \xi_{\tau n}\right)^{(h)}\right)^{\eta_{\tau \rho}}$ with $\eta_{\tau \rho}>0$. Since $\widetilde{F}$ and $G$ have the same factors $\left(u_{00}+\right.$ $\left.u_{01} \xi_{\tau 1}+\cdots+u_{0 n} \xi_{\tau n}\right)^{(h)}$ and the primitive factor of $\widetilde{F}$ w.r.t. $u_{00}^{(h)}$ is itself, thus we can find $\eta_{\tau \rho}$ such that $\widetilde{F}=G$, which completes the proof.

5.2. Differential Chow quasi-variety for a differential algebraic cycle. A $\delta$-cycle $V$ in the $n$-dimensional $\delta$-affine space with dimension $d$, order $h$, leading $\delta$-degree $g$, and $\delta$-degree $m$ is said to be of index $(n, d, h, g, m)$. In this section, we will define the $\delta$-Chow quasi-variety in certain cases such that each point in this $\delta$-variety represents a $\delta$-cycle with a given index $(n, d, h, g, m)$.

For a given index $(n, d, h, g, m)$, a $\delta$-polynomial $F\left(\mathbf{u}_{0}, \ldots, \mathbf{u}_{d}\right)$ which has unknown coefficients $a_{\lambda}(\lambda=0, \ldots, D)$ and satisfies the following two conditions is referred to as a $\delta$-polynomial with index $(n, d, h, g, m)$. 
1) $F$ is a homogenous polynomial of the same degree $m$ in each set of indeterminates $\mathbf{u}_{i}=\left(u_{i 0}, u_{i 1}, \ldots, u_{i n}\right)(i=0, \ldots, d)$ and their derivatives. Furthermore, for each $u_{i j}, \operatorname{ord}\left(F, u_{i j}\right)$ is either $h$ or $-\infty$. In particular, $\operatorname{ord}\left(F, u_{00}\right)=h$.

2) As a polynomial in $u_{00}^{(h)}, u_{01}^{(h)}, \ldots, u_{0 n}^{(h)}$, its total degree is $g$. In particular, $\operatorname{deg}\left(F, u_{00}^{(h)}\right)=g$.

We want to determine the necessary and sufficient conditions imposed on $a_{\lambda}(\lambda=$ $0, \ldots, D)$ in order that $F$ be the Chow form for a $\delta$-cycle with index $(n, d, h, g, m)$. Proceeding in this way, if the necessary and sufficient conditions given in Theorem 5.1 can be expressed by some $\delta$-polynomials in $a_{\lambda}$, then the $\delta$-variety defined by them is called the $\delta$-Chow (quasi)-variety. More precisely, we have the following definition.

Definition 5.6. Let $F\left(\mathbf{u}_{0}, \ldots, \mathbf{u}_{d}\right)$ be a $\delta$-polynomial with $\delta$-F $\mathcal{F}$-indeterminates $a_{i}(i=0, \ldots, D)$ in $\mathcal{E}$ as coefficients and with index $(n, d, h, g, m)$. A quasi- $\delta$ variety $\mathbb{C V}$ in the variables $a_{i}$ is called the differential Chow quasi-variety with index $(n, d, h, g, m)$ if a point $\bar{a}_{i}$ is in $\mathbb{C V}$ if and only if $\widetilde{F}$ is the Chow form for a $\delta$-cycle with index $\left(n, d, h, g, m_{1}\right)$ with $m_{1} \leq m$, where $\widetilde{F}$ is obtained from $F$ by first replacing $a_{i}$ by $\bar{a}_{i}$ and then taking the primitive part with respect to the variable $u_{00}^{(h)}$.

In the case $h=0$, since Theorems 1.2 and 5.1 become their algebraic counterparts, we can obtain the equations for the algebraic Chow variety in the same way as in [16, pp.56-57]. So in the following, we only consider the case $h>0$. For $h>0$, the case $g=1$ is relatively simple. The following result shows how to determine the defining equations for the $\delta$-Chow quasi-variety with index $(n, d, h, g, m)$ in the case $g=1$.

Theorem 5.7. Let $F\left(\mathbf{u}_{0}, \ldots, \mathbf{u}_{d}\right)$ be a $\delta$-polynomial with $\delta$-F-indeterminates $a_{\nu}$ $(\nu=0, \ldots, D)$ as coefficients and with index $(n, d, h, g, m)$ with $g=1$. Let $I_{F}$ be the initial of $F$ w.r.t. the elimination ranking $u_{00} \succ u_{i, j}$ and $a_{0}, \ldots, a_{I}$ be the coefficients of $I_{F}$. Then we can find a set of $\delta$-homogeneous $\delta$-polynomials

$$
R_{\omega}\left(a_{0}, \ldots, a_{D}\right)(\omega=1, \ldots, v)
$$

in $a_{\nu}$ such that $\mathbb{V}\left(R_{\omega}: \omega=1, \ldots, v\right) \backslash \mathbb{V}\left(a_{0}, \ldots, a_{I}\right)$ is the $\delta$-Chow quasi-variety of index $(n, d, h, g, m)$ with $g=1$.

Proof. In order for $F$ to be a differential Chow form, by Theorem [1.2, $F$ must be $\delta$-homogeneous in each $\mathbf{u}_{i}$. Let $\lambda$ be a $\delta$ - $\mathcal{F}$-indeterminate. For each $i$, replacing $\mathbf{u}_{i}$ by $\lambda \mathbf{u}_{i}$ in $F$, we should have

$$
F\left(\mathbf{u}_{0}, \ldots, \mathbf{u}_{i-1}, \lambda \mathbf{u}_{i}, \mathbf{u}_{i+1}, \ldots, \mathbf{u}_{d}\right)=\lambda^{m} F\left(\mathbf{u}_{0}, \ldots, \mathbf{u}_{d}\right) .
$$

Comparing the coefficients of the power products of $\lambda, u_{i j}$ and their derivatives, we obtain a system of linearly homogenous equations $R_{\omega}\left(a_{0}, \ldots, a_{D}\right)=0(\omega=$ $\left.1, \ldots, e_{1}\right)$ in $a_{\nu}$, which are the conditions for $F$ to be $\delta$-homogeneous and with degree $m$ in each $\mathbf{u}_{i}$. So by Gaussian elimination in linear algebra, we can obtain a basis for the solution space of $R_{\omega}=0\left(\omega=1, \ldots, e_{1}\right)$. More precisely, if the coefficient matrix of this linear equation is of rank $r$, then $r$ of $\left\{a_{0}, \ldots, a_{D}\right\}$ are the linear combinations of the other $D+1-r$ of $a_{\nu}$. Now substitute these $r$ relations into $F$ and denote the new $\delta$-polynomial by $F_{1}$. That is, $F_{1}$ is a $\delta$-homogenous $\delta$-polynomial in each $\mathbf{u}_{i}$ which only involves $D+1-r$ independent coefficients $a_{\nu}$. 
Since $g=1, F_{1}$ can be written in the form

$$
F_{1}\left(\mathbf{u}_{0}, \ldots, \mathbf{u}_{d}\right)=A_{0} u_{00}^{(h)}+A_{1} u_{01}^{(h)}+\cdots+A_{n} u_{0 n}^{(h)}+B,
$$

where $A_{i}$ and $B$ are free of $u_{0 k}^{(h)}$. Denote $-\left(A_{1} u_{01}^{(h)}+\cdots+A_{n} u_{0 n}^{(h)}+B\right) / A_{0}$ by $\gamma$. Then $u_{00}^{(h)}=\gamma$ is the solution of $F_{1}$ as an algebraic polynomial in $u_{00}^{(h)}$. Let $\xi_{j}=\frac{\partial F_{1}}{\partial u_{0 j}^{(h)}} /\left.\frac{\partial F_{1}}{\partial u_{00}^{(h)}}\right|_{u_{00}^{(h)}=\gamma}=A_{j} /\left.A_{0}\right|_{u_{00}^{(h)}=\gamma}$ for $j=1, \ldots, n$. Proceeding as in the proof of Theorem 4.27, we define the derivatives of $\xi_{j}$ to be $\xi_{j}^{(k)}=\left.\left(\delta \xi_{j}^{(k-1)}\right)\right|_{u_{00}^{(h)}=\gamma}$. It is easy to see that this definition is well defined. Since $F_{1}$ is $\delta$-homogeneous in $\mathbf{u}_{0}$, by Theorem 4.16, for $r \neq 0$,

$$
\sum_{j=0}^{n} \sum_{k \geq 0}\left(\begin{array}{c}
k+r \\
r
\end{array}\right) u_{0 j}^{(k)} \frac{\partial F_{1}}{\partial u_{0 j}^{(k+r)}}=0 .
$$

In the case $r=h$, we have $\sum_{j=0}^{n} u_{0 j} \frac{\partial F_{1}}{\partial u_{0 j}^{(h)}}=0$. Set $u_{00}^{(h)}=\gamma$ in the identity $\sum_{j=0}^{n} u_{0 j} \frac{\partial F_{1}}{\partial u_{0 j}^{(h)}}=0$; then we have $u_{00}+\sum_{j=1}^{n} u_{0 j} \xi_{j}=0$ with $u_{00}^{(h)}=\gamma$. So $\left(\xi_{1}, \ldots, \xi_{n}, \ldots, \xi_{1}^{(h-1)}, \ldots, \xi_{n}^{(h-1)}\right)$ is a solution of ${ }^{a} \mathbb{P}_{0},{ }^{a} \mathbb{P}_{0}^{\prime}, \ldots,{ }^{a} \mathbb{P}_{0}^{(h-1)}$ and $\gamma=$ $-\left(\sum_{j=1}^{n} u_{0 j} \xi_{j}\right)^{(h)}$. So $F_{1}\left(\mathbf{u}_{0}, \ldots, \mathbf{u}_{d}\right)=A_{0}\left(u_{00}+\sum_{j=1}^{n} u_{0 j} \xi_{j}\right)^{(h)}$. As a consequence, with these $\xi_{i}$, the second condition and the second part of the third condition in Theorem 5.1 are satisfied.

In order for $F_{1}$ to be the Chow form for some $\delta$-variety, by Theorem 5.1 , $\left(\xi_{1}, \ldots, \xi_{n}\right)$ should satisfy $\mathbb{P}_{\sigma}=0(\sigma=1, \ldots, d)$ and $F_{1}\left(S^{0} \xi, \ldots, S^{d} \xi\right)=0$, where $S^{i}$ are $(n+1) \times(n+1)$-skew symmetric matrices with elements independent indeterminates and $\xi=\left(1, \xi_{1}, \ldots, \xi_{n}\right)^{T}$.

First, setting $y_{j}=A_{j} / A_{0}$ in $\mathbb{P}_{\sigma}=0$, we get $u_{\sigma 0} A_{0}+\sum_{j=1}^{n} u_{\sigma j} A_{j}=0$. Then we obtain some equations in $a_{\nu}$ by equating to zero the coefficients of the various $\delta$-products of $\mathbf{u}_{0}, \ldots, \mathbf{u}_{d}$. This gives $\delta$-polynomials $R_{\omega}\left(a_{0}, \ldots, a_{D}\right)\left(\omega=e_{1}+\right.$ $\left.1, \ldots, e_{2}\right)$.

Second, we obtain some $\delta$-equations $\chi_{\tau}\left(a_{\nu}, y_{1}, \ldots, y_{n}\right)$ by equating to zero the coefficients of all $\delta$-products of the independent indeterminates $s_{j k}^{i}(j>k)$ in $F_{1}\left(S^{0} Y, \ldots, S^{d} Y\right)=0$ with $Y=\left(1, y_{1}, \ldots, y_{n}\right)^{T}$. Then setting $y_{j}^{(k)}=\xi_{j}^{(k)}$ in the above $\chi_{\tau}$ and clearing denominators, we obtain polynomial equations $p_{\mu}$ in $u_{i j}^{(k)}$ and $a_{\nu}$. Equating to zero the coefficients of the power products of the $u_{i j}^{(k)}$ in $p_{\mu}$, we finally obtain $\delta$-polynomials in $a_{\nu}: R_{\omega}\left(a_{0}, \ldots, a_{D}\right)\left(\omega=e_{2}+1, \ldots, v\right)$. We then obtain the defining equations $R_{\omega}=0$ for the Chow variety.

We now show that all the $R_{\omega}$ are $\delta$-homogenous $\delta$-polynomials. Denote $\mathbf{a}=$ $\left(a_{0}, \ldots, a_{D}\right)$. We have known for $\omega=1, \ldots, e_{1}$ that $R_{\omega}$ are linearly homogenous $\delta$-polynomials in a. Since $F_{1}$ as well as $A_{i}$ are linearly homogenous in a, $R_{\omega}(\mathbf{a})\left(\omega=e_{1}+1, \ldots, e_{2}\right)$ are linearly homogenous $\delta$-polynomials. To show $R_{\omega}(\mathbf{a})\left(\omega=e_{2}+1, \ldots, v\right)$ are $\delta$-homogenous $\delta$-polynomials, by induction on $k$, we first show that for any $\delta$ - $\mathcal{F}$-indeterminate $\lambda$ and for each $j$ and $k, \xi_{j}^{(k)}(\lambda \mathbf{a})=\xi_{j}^{(k)}(\mathbf{a})$. By the expression of $\gamma$, it is clear that $\gamma(\lambda \mathbf{a})=\gamma$ since $\xi_{j}=A_{j} /\left.A_{0}\right|_{u_{00}^{(h)}=\gamma}$ and $\xi_{j}(\lambda \mathbf{a})=\left.\frac{A_{j}(\lambda \mathbf{a})}{A_{0}(\lambda \mathbf{a})}\right|_{u_{00}^{(h)}=\gamma(\lambda \mathbf{a})}=\left.\frac{\lambda A_{j}(\mathbf{a})}{\lambda A_{0}(\mathbf{a})}\right|_{u_{00}^{(h)}=\gamma}=\xi_{j}(\mathbf{a})$. So it holds for $k=0$. Suppose it holds for $k-1$, that is, $\xi_{j}^{(k-1)}(\lambda \mathbf{a})=\xi_{j}^{(k-1)}(\mathbf{a})$. Since $\xi_{j}^{(k)}=\left.\left(\delta \xi_{j}^{(k-1)}\right)\right|_{u_{00}^{(h)}=\gamma}$, 
$\xi_{j}^{(k)}(\lambda \mathbf{a})=\left.\left(\delta\left(\xi_{j}^{(k-1)}(\lambda \mathbf{a})\right)\right)\right|_{u_{00}^{(h)}=\gamma(\lambda \mathbf{a})}=\left.\left(\delta \xi_{j}^{(k-1)}(\mathbf{a})\right)\right|_{u_{00}^{(h)}=\gamma(\mathbf{a})}=\xi_{j}^{(k)}(\mathbf{a})$. Now we are going to show that $R_{\omega}(\mathbf{a})\left(\omega=e_{2}+1, \ldots, v\right)$ are $\delta$-homogenous $\delta$-polynomials. Since $F_{1}$ is linearly homogenous in $\mathbf{a}$, each $\chi_{\tau}\left(a_{\nu}, y_{1}, \ldots, y_{n}\right)$ is linearly homogenous in a. Setting $y_{j}^{(k)}=\xi_{j}^{(k)}$ in $\chi_{\tau}$, it is clear that $\chi_{\tau}\left(a_{\nu}, \xi_{1}, \ldots, \xi_{n}\right)(\lambda \mathbf{a})=$ $\lambda \cdot \chi_{\tau}\left(a_{\nu}, \xi_{1}, \ldots, \xi_{n}\right)$ and the denominator of $\chi_{\tau}\left(a_{\nu}, \xi_{1}, \ldots, \xi_{n}\right)$ is a pure algebraic homogenous polynomial in a. Thus, $p_{\mu}$ is $\delta$-homogenous in a and the $\delta$-homogeneity of $R_{\omega}$ follows.

Let $a_{0}, \ldots, a_{I}$ be the coefficients of $I_{F}$. Then we claim that the quasi-projective $\delta$-variety $\mathbb{C} \mathbb{V}=\mathbb{V}\left(R_{\omega}: \omega=1, \ldots, v\right) \backslash \mathbb{V}\left(a_{0}, \ldots, a_{I}\right)$ is the $\delta$-Chow quasi-variety. Indeed, for every element $\left(\overline{a_{0}}, \ldots, \overline{a_{D}}\right)$ in $\mathbb{C V}$, following the proof of this theorem, $\bar{F}$ with coefficients $\overline{a_{\nu}}$ satisfies the four conditions in Theorem [5.1. And since $g=1$, its primitive part must be irreducible and satisfy the four conditions too, which consequently must be the Chow form for some irreducible $\delta$-variety with index $\left(n, d, h, 1, m_{1}\right)$ with $m_{1} \leq m$.

The following example illustrates the procedure to compute the $\delta$-Chow quasivariety in the case of $g=1$.

Example 5.8. We consider a $\delta$-polynomial which has 16 terms and has index $(2,1,1,1,2)$ to illustrate the proof of Theorem 5.7. $F=a_{1} u_{12}^{2} u_{01} u_{00}^{\prime}+a_{2} u_{11} u_{12} u_{02} u_{00}^{\prime}$ $+a_{3} u_{01} u_{02} u_{12} u_{10}^{\prime}+a_{4} u_{02}^{2} u_{11} u_{10}^{\prime}+a_{5} u_{12}^{2} u_{00} u_{01}^{\prime}+a_{6} u_{10} u_{12} u_{02} u_{01}^{\prime}+a_{7} u_{00} u_{02} u_{12} u_{11}^{\prime}+$ $a_{8} u_{02}^{2} u_{10} u_{11}^{\prime}+a_{9} u_{10} u_{01} u_{12} u_{02}^{\prime}+a_{10} u_{00} u_{02} u_{11} u_{12}^{\prime}+a_{11} u_{11} u_{12} u_{00} u_{02}^{\prime}+a_{12} u_{01} u_{02} u_{10} u_{12}^{\prime}$ $+a_{13} u_{00} u_{11}^{2} u_{02}+a_{14} u_{00} u_{01} u_{11} u_{12}+a_{15} u_{01} u_{10} u_{02} u_{11}+a_{16} u_{10} u_{01}^{2} u_{12}$. We will derive the conditions about the coefficients $a_{\nu}$ under which $F$ is a Chow form. First, in order for $F$ to be $\delta$-homogenous, we have $R_{1}=a_{5}+a_{1}, R_{2}=a_{8}+a_{4}, R_{3}=$ $a_{9}+a_{6}, R_{4}=a_{10}+a_{7}, R_{5}=a_{11}+a_{2}, R_{6}=a_{12}+a_{3}$. Replace $a_{5}, a_{8}, a_{9}, a_{10}, a_{11}, a_{12}$ by $-a_{1},-a_{4},-a_{6},-a_{7},-a_{2},-a_{3}$ respectively in $F$ to obtain $F_{1}$.

For such an $F_{1}, A_{0}=a_{1} u_{12}^{2} u_{01}+a_{2} u_{11} u_{12} u_{02}, A_{1}=-a_{1} u_{12}^{2} u_{00}+a_{6} u_{10} u_{12} u_{02}, A_{2}$ $=-a_{6} u_{10} u_{01} u_{12}-a_{2} u_{11} u_{12} u_{00}$, and $B=a_{3} u_{01} u_{02} u_{12} u_{10}^{\prime}+a_{4} u_{02}^{2} u_{11} u_{10}^{\prime}+a_{7} u_{00} u_{02} u_{12}$ $u_{11}^{\prime}-a_{4} u_{02}^{2} u_{10} u_{11}^{\prime}-a_{7} u_{00} u_{02} u_{11} u_{12}^{\prime}-a_{3} u_{01} u_{02} u_{10} u_{12}^{\prime}+a_{13} u_{00} u_{11}^{2} u_{02}+a_{14} u_{00} u_{01} u_{11}$ $u_{12}+a_{15} u_{01} u_{10} u_{02} u_{11}+a_{16} u_{10} u_{01}^{2} u_{12}$. Then $\gamma=\left(F_{1}-A_{0} u_{00}^{\prime}\right) / A_{0}, \xi_{1}=A_{1} /\left.A_{0}\right|_{u_{00}^{\prime}=\gamma}$, and $\xi_{2}=A_{2} /\left.A_{0}\right|_{u_{00}^{\prime}=\gamma}$. To confirm that $u_{10}+u_{11} \xi_{1}+u_{12} \xi_{2}=0$, we must have $R_{7}=a_{6}-a_{1}=0, R_{8}=a_{2}+a_{1}=0$.

In order to satisfy the fourth condition of Theorem 5.1 we obtain a set of $\delta$ polynomial equations $R_{\omega}\left(a_{0}, \ldots, a_{16}\right)=0$ which have more complicated forms. By simplifying them with $R_{7}=0$ and $R_{8}=0$, we obtain $R_{9}=a_{7} a_{1}\left(a_{15}+a_{16}\right), R_{10}=$ $a_{7} a_{1}\left(a_{14}+a_{16}\right), R_{11}=a_{7} a_{1}\left(a_{13}-a_{16}\right), R_{12}=a_{1}\left(a_{4}-a_{7}\right), R_{13}=a_{1}\left(a_{3}+a_{7}\right)$, $R_{14}=a_{1} a_{16}\left(a_{1}-a_{7}\right), R_{15}=a_{15} a_{1}^{2}+a_{1} a_{7} a_{16}, R_{16}=a_{14} a_{1}^{2}+a_{1} a_{7} a_{16}, R_{17}=$ $a_{7} a_{1}\left(a_{1}-a_{7}\right), R_{18}=a_{1}^{2} a_{13}-a_{1} a_{7} a_{16}, R_{19}=a_{1}^{3}-a_{7}^{2} a_{1}$. Thus the Chow quasivariety is $\mathbb{V}\left(R_{1}, \ldots, R_{19}\right) / \mathbb{V}\left(a_{1}, a_{2}\right)=\mathbb{V}\left(a_{2}+a_{1}, a_{3}+a_{1}, a_{4}-a_{1}, a_{5}+a_{1}, a_{6}-a_{1}, a_{7}-\right.$ $\left.a_{1}, a_{8}+a_{1}, a_{9}+a_{1}, a_{10}+a_{1}, a_{11}-a_{1}, a_{12}-a_{1}, a_{14}+a_{13}, a_{15}+a_{13}, a_{16}-a_{13}\right) / \mathbb{V}\left(a_{1}\right)$. From Example 4.6] it is easy to check that each point of this quasi-variety is the coefficient of the Chow form for $\mathbb{V}\left(a_{1} y_{1}^{\prime}+a_{13} y_{2}\right)$ for some $a_{1}, a_{13} \in \mathcal{F}$. Note that $a_{13}$ could be zero and the result is still valid.

We are unable to prove the existence of the Chow quasi-variety in the case of $g>1$. The main difficulty is how to perform elimination for a mixed system consisting of both differential and algebraic equations. In our case, condition 2 and the second part of condition 3 of Theorem 5.1 generate algebraic equations 
in the coefficients of $F$ and $\xi_{i j}$, while condition 1, the first part of condition 3, and condition 4 of Theorem 5.1 generate $\delta$-equations. Also, we need to eliminate variables $\xi_{i j}$ from these equations.

The following example shows that the $\delta$-Chow quasi-variety can be easily defined in the case of $n=1$.

Example 5.9. If $n=1$, then $d=0$, and every irreducible $\delta$-homogeneous $\delta$ polynomial in $\mathbf{u}_{0}=\left(u_{00}, u_{01}\right)$ is the differential Chow form for some irreducible $\delta$-variety.

Proof. Let $F\left(\mathbf{u}_{0}\right)=F\left(u_{00}, u_{01}\right)$ be an irreducible $\delta$-homogenous $\delta$-polynomial with degree $m$ and order $h$. Then $F\left(-\frac{u_{00}}{u_{01}},-1\right)=\left(-\frac{1}{u_{01}}\right)^{m} F\left(u_{00}, u_{01}\right)$. Let $g\left(-\frac{u_{00}}{u_{01}}\right)=$ $F\left(-\frac{u_{00}}{u_{01}},-1\right)$. It is easy to show that $g(y)$ is an irreducible $\delta$-polynomial. By Example 4.6, the Chow form of the prime $\delta$-ideal $\operatorname{sat}(g(y))$ is $\left(-u_{01}\right)^{m} g\left(-\frac{u_{00}}{u_{01}}\right)=$ $\left(-u_{01}\right)^{m} F\left(-\frac{u_{00}}{u_{01}},-1\right)=F\left(u_{00}, u_{01}\right)$, and the result is proved.

As a consequence, the Chow quasi-variety in the case of $n=1$ always exists.

Example 5.10. Let $\mathbf{u}_{0}=\left(u_{00}, u_{01}\right)$ and $F\left(\mathbf{u}_{0}\right)$ be a homogenous $\delta$-polynomial with index $(1,0, h, g, m)$ and coefficients $a_{0}, \ldots, a_{D}$. Let $I_{F}$ be the initial of $F$ w.r.t. the elimination ranking $u_{00} \succ u_{01}$ and $a_{0}, \ldots, a_{I}$ the coefficients of $I_{F}$. Let $R_{\omega}\left(a_{0}, \ldots, a_{D}\right)\left(\omega=1, \ldots, e_{1}\right)$ be the equations obtained in the proof of Theorem 5.7. Then under the condition $R_{\omega}\left(a_{0}, \ldots, a_{D}\right)=0\left(\omega=1, \ldots, e_{1}\right), F$ will become a $\delta$-homogenous $\delta$-polynomial. Then by Example [5.9] $\mathbb{V}\left(R_{\omega}: \omega=1, \ldots, e_{1}\right) \backslash$ $\mathbb{V}\left(a_{0}, \ldots, a_{I}\right)$ is the $\delta$-Chow quasi-variety with index $(1,0, h, g, m)$.

\section{Generalized differential Chow form and Differential Resultant}

We mentioned that the differential Chow form can be obtained by intersecting the $\delta$-variety with generic $\delta$-hyperplanes. In this section, we show that when intersecting an irreducible $\delta$-variety of dimension $d$ by $d+1$ generic $\delta$-hypersurfaces, we can obtain the generalized Chow form which has similar properties to the Chow form. As a direct consequence, we can define the differential resultant and obtain its properties.

6.1. Generalized differential Chow form. Let $V \subset \mathcal{E}^{n}$ be an irreducible $\delta$ - $\mathcal{F}$ variety with dimension $d$ and order $h$, and

$$
\mathbb{P}_{i}=u_{i 0}+\sum_{j=1}^{n} \sum_{k=0}^{s_{i}} u_{i j k} y_{j}^{(k)}+\sum_{\substack{\alpha \in \mathbb{Z}_{\geq 0}^{n\left(s_{i}+1\right)} \\ 1<|\alpha| \leq m_{i}}} u_{i \alpha}\left(\mathbb{Y}^{\left(s_{i}\right)}\right)^{\alpha} \quad(i=0, \ldots, d)
$$

be a generic $\delta$-polynomial of order $s_{i} \geq 0$ and degree $m_{i} \geq 1$, where $u_{i 0}, u_{i j k}, u_{i \alpha}$ $\left(i=0, \ldots, d ; j=1, \ldots, n ; k=0, \ldots, s_{i} ; \alpha \in \mathbb{Z}_{>0}^{n\left(s_{i}+1\right)}, 1<|\alpha| \leq m_{i}\right)$ are $\delta$ - $\mathcal{F}$ indeterminates in $\mathcal{E}$ and $\left(\mathbb{Y}^{\left[s_{i}\right]}\right)^{\alpha}$ is a monomial in $\mathcal{F}\left[\mathbb{Y}^{\left[s_{i}\right]}\right]$ with exponent vector $\alpha=\left(\alpha_{10}, \ldots, \alpha_{n 0}, \alpha_{11}, \ldots, \alpha_{n 1}, \ldots, \alpha_{1 s_{i}}, \ldots, \alpha_{n s_{i}}\right)$, i.e. $\left(\mathbb{Y}^{\left[s_{i}\right]}\right)^{\alpha}=\prod_{j=1}^{n} \prod_{k=0}^{s_{i}}\left(y_{j}^{(k)}\right)^{\alpha_{j k}}$ and $|\alpha|=\sum_{j=1}^{n} \sum_{k=0}^{s_{i}} \alpha_{j k}$. For convenience in the rest of the paper, we denote the nonlinear part of each $\mathbb{P}_{i}$ by $f_{i}$, that is,

$$
\mathbb{P}_{i}=u_{i 0}+\sum_{j=1}^{n} \sum_{k=0}^{s_{i}} u_{i j k} y_{j}^{(k)}+f_{i} \quad(i=0, \ldots, d) .
$$


Denote $\mathbf{u}$ to be the set consisting of all the $u_{i j k}$ and $u_{i \alpha}$ for $i=0, \ldots, d$. Let $\xi=\left(\xi_{1}, \ldots, \xi_{n}\right) \subset \mathcal{E}^{n}$ be a generic point of $V$, which is free from $\mathcal{F}\left\langle\mathbf{u}, u_{00}, \ldots, u_{d 0}\right\rangle$. We define $d+1$ elements $\zeta_{0}, \zeta_{1}, \ldots, \zeta_{d}$ of $\mathcal{F}\left\langle\mathbf{u}, \xi_{1}, \ldots, \xi_{n}\right\rangle$ :

$$
\zeta_{i}=-\sum_{j=1}^{n} \sum_{k=0}^{s_{i}} u_{i j k} \xi_{j}^{(k)}-f_{i}\left(\xi_{1}, \ldots, \xi_{n}\right) \quad(i=0, \ldots, d) .
$$

Similar to the proof of Lemma 4.1, we can prove that if $d>0$, then any set of $d$ elements of $\zeta_{0}, \ldots, \zeta_{d}$ is a $\delta$-transcendence basis of $\mathcal{F}\langle\mathbf{u}\rangle\left\langle\zeta_{0}, \ldots, \zeta_{d}\right\rangle$ over $\mathcal{F}\langle\mathbf{u}\rangle$. If $d=0, \zeta_{0}$ is $\delta$-algebraic over $\mathcal{F}\langle\mathbf{u}\rangle$. We thus have

Lemma 6.1. d.tr.deg $\mathcal{F}\langle\mathbf{u}\rangle\left\langle\zeta_{0}, \ldots, \zeta_{d}\right\rangle / \mathcal{F}\langle\mathbf{u}\rangle=d$.

Let $\mathbb{I}_{\zeta}$ be the prime $\delta$-ideal in $\mathcal{R}=\mathcal{F}\langle\mathbf{u}\rangle\left\{z_{0}, \ldots, z_{d}\right\}$ having $\zeta=\left(\zeta_{0}, \ldots, \zeta_{d}\right)$ as a generic point. By Lemma 6.1, the dimension of $\mathbb{I}_{\zeta}$ is $d$. Then, the characteristic set of $\mathbb{I}_{\zeta}$ w.r.t. any ranking consists of an irreducible $\delta$-polynomial $g\left(z_{0}, \ldots, z_{d}\right)$ in $\mathcal{R}$ and $\mathbb{I}_{\zeta}=\operatorname{sat}(g)$. Since the coefficients of $g\left(z_{0}, \ldots, z_{d}\right)$ are elements in $\mathcal{F}\langle\mathbf{u}\rangle$, without loss of generality we assume that $g\left(\mathbf{u} ; z_{0}, \ldots, z_{d}\right)$ is irreducible in $\mathcal{F}\left\{\mathbf{u} ; z_{0}, \ldots, z_{d}\right\}$. We shall subsequently replace $z_{0}, \ldots, z_{d}$ by $u_{00}, \ldots, u_{d 0}$, and obtain

$$
G\left(\mathbf{u}_{0}, \mathbf{u}_{1}, \ldots, \mathbf{u}_{d}\right)=g\left(\mathbf{u} ; u_{00}, \ldots, u_{d 0}\right),
$$

where $\mathbf{u}_{i}=\left(u_{i 0}, \ldots, u_{i j k}, \ldots, u_{i \alpha}, \ldots\right)$ is the sequence of the coefficients of $\mathbb{P}_{i}$.

Definition 6.2. The $\delta$-polynomial defined in (6.3) is called the generalized Chow form of $V$ or the prime $\delta$-ideal $\mathbb{I}(V)$ with respect to $\mathbb{P}_{i}(i=0, \ldots, d)$.

Similar to Theorem 4.17, we can prove that the generalized Chow form is a $\delta$ homogeneous $\delta$-polynomial in each set of indeterminates $\mathbf{u}_{i}$, but in this case the homogeneous degree for distinct $\mathbf{u}_{i}$ may be distinct. The order of the generalized Chow form w.r.t. $\mathbf{u}_{i}$, denoted by $\operatorname{ord}\left(G, \mathbf{u}_{i}\right)$, is defined to be $\max _{u \in \mathbf{u}_{i}} \operatorname{ord}(g, u)$. Now we will consider the order of the generalized Chow form.

Theorem 6.3. Let $\mathcal{I}$ be a prime $\delta$-ideal with dimension $d$ and order $h$ defined over $\mathcal{F}$, and $G\left(\mathbf{u}_{0}, \mathbf{u}_{1}, \ldots, \mathbf{u}_{d}\right)=g\left(\mathbf{u} ; u_{00}, u_{10}, \ldots, u_{d 0}\right)$ be its generalized Chow form. Then for a fixed $i$ between 0 and $d$, ord $\left(g, u_{i 0}\right)=h+s-s_{i}$ with $s=\sum_{l=0}^{d} s_{l}$. Moreover, $\operatorname{ord}\left(G, \mathbf{u}_{i}\right)=h+s-s_{i}$.

Proof. Use the notation as above in this section. Let $\mathcal{I}_{d}=\left[\mathcal{I}, \mathbb{P}_{0}, \ldots, \mathbb{P}_{i-1}, \mathbb{P}_{i+1}\right.$, $\left.\ldots, \mathbb{P}_{d}\right] \subset \mathcal{F}\left\langle\mathbf{u}_{0}, \ldots, \mathbf{u}_{i-1}, \mathbf{u}_{i+1}, \ldots, \mathbf{u}_{d}\right\rangle\left\{y_{1}, \ldots, y_{n}\right\}$. By Theorem 3.13, $\mathcal{I}_{d}$ is a prime $\delta$-ideal with dimension 0 and order $h+s_{0}+\cdots+s_{i-1}+s_{i+1}+\cdots+s_{d}=h+s-s_{i}$, where $s=\sum_{l=0}^{d} s_{l}$.

Let $\mathbb{I}_{\zeta, \xi}=\left[\mathcal{I}, \mathbb{P}_{0}, \ldots, \mathbb{P}_{d}\right] \subset \mathcal{F}\langle\mathbf{u}\rangle\left\{u_{00}, \ldots, u_{d 0}, y_{1}, \ldots, y_{n}\right\}$ and $\mathcal{I}_{1}=\left[\mathcal{I}, \mathbb{P}_{0}, \ldots\right.$, $\left.\mathbb{P}_{d}\right]=\left[\mathcal{I}_{d}, \mathbb{P}_{0}\right] \subset \mathcal{F}\left\langle\widehat{\mathbf{u}} \backslash\left\{u_{i 0}\right\}\right\rangle\left\{u_{i 0}, y_{1}, \ldots, y_{n}\right\}$, where $\widehat{\mathbf{u}}=\mathbf{u}_{0} \cup \cdots \cup \mathbf{u}_{d}$. Denote $\operatorname{ord}\left(G, u_{i 0}\right)$ by $h_{1}$. Similar to the proof of Lemma 4.10, we can show that $\mathcal{A}=$ $g\left(\mathbf{u} ; u_{00}, \ldots, u_{d 0}\right), \frac{\partial g}{\partial u_{i 0}^{\left(h_{1}\right)}} y_{1}-\frac{\partial g}{\partial u_{i 10}^{\left(h_{1}\right)}}, \ldots, \frac{\partial g}{\partial u_{i 0}^{\left(h_{1}\right)}} y_{n}-\frac{\partial g}{\partial u_{i n 0}^{\left(h_{1}\right)}}$ is a characteristic set of $\mathbb{I}_{\zeta, \xi}$ w.r.t. the elimination ranking $u_{00} \prec \cdots \prec u_{i-1,0} \prec u_{i+1,0} \prec u_{d 0} \prec u_{i 0} \prec y_{1} \prec \cdots \prec$ $y_{n}$. Clearly, $\mathcal{I}_{1}$ is the $\delta$-ideal generated by $\mathbb{I}_{\zeta, \xi}$ in $\mathcal{F}\left\langle\widehat{\mathbf{u}} \backslash\left\{u_{i 0}\right\}\right\rangle\left\{u_{i 0}, y_{1}, \ldots, y_{n}\right\}$. Since $\left\{u_{00}, \ldots, u_{i-1,0}, u_{i+1,0}, \ldots, u_{d 0}\right\}$ is a parametric set of $\mathbb{I}_{\zeta, \xi}, \mathcal{A}$ is also a characteristic set of $\mathcal{I}_{1}$ w.r.t. the elimination ranking $u_{i 0} \prec y_{1} \prec \cdots \prec y_{n}$. Since $\operatorname{dim}\left(\mathcal{I}_{1}\right)=0$, from Corollary 2.10 we have $\operatorname{ord}\left(\mathcal{I}_{1}\right)=\operatorname{ord}(\mathcal{A})=\operatorname{ord}\left(g, u_{i 0}\right)$.

On the other hand, let $\mathcal{I}_{1}^{(l)}=\left[\mathcal{I}_{d}, u_{i 0}^{(l)}+\sum_{j=1}^{n} \sum_{k=0}^{s_{i}} u_{i j k} y_{j}^{(k)}+f_{i}\right] \subset \mathcal{F}\langle\widehat{\mathbf{u}} \backslash$ $\left.\left\{u_{i 0}\right\}\right\rangle\left\{u_{i 0}, y_{1}, \ldots, y_{n}\right\}\left(l=1, \ldots, s_{i}\right)$. Since $\operatorname{dim}\left(\mathcal{I}_{1}^{(l)}\right)=0, u_{i 0}$ is a leading variable 
of $\mathcal{I}_{1}^{(l)}$ for any ranking. Thus, by Lemma 3.11, we have ord $\left(\mathcal{I}_{1}^{(l+1)}\right)=\operatorname{ord}\left(\mathcal{I}_{1}^{(l)}\right)+1$, which follows that $\operatorname{ord}\left(\mathcal{I}_{1}^{\left(s_{i}\right)}\right)=\operatorname{ord}\left(\mathcal{I}_{1}\right)+s_{i}$, and it is easy to see that $\operatorname{ord}\left(\mathcal{I}_{1}^{\left(s_{i}\right)}\right)=$ $\operatorname{ord}\left(\mathcal{I}_{d}\right)+s_{i}$. Indeed, let $\mathcal{A}$ be a characteristic set of $\mathcal{I}_{d}$ w.r.t. some orderly ranking $\mathscr{R}$, and let $t$ be the pseudo remainder of $u_{i 0}^{\left(s_{i}\right)}+\sum_{j=1}^{n} \sum_{k=0}^{s_{i}} u_{i j k} y_{j}^{(k)}+f_{i}$ w.r.t. $\mathcal{A}$ under the ranking $\mathscr{R}$. Clearly, $\operatorname{ord}\left(t, u_{i 0}\right)=s_{i}$. It is obvious that for some orderly ranking, $\{\mathcal{A}, t\}$ is a characteristic set of $\mathcal{I}_{1}^{\left(s_{i}\right)}$ with $\operatorname{ld}(\mathcal{A})$ and $u_{i 0}^{\left(s_{i}\right)}$ as its leaders, so $\operatorname{ord}\left(\mathcal{I}_{1}^{\left(s_{i}\right)}\right)=\operatorname{ord}\left(\mathcal{I}_{d}\right)+s_{i}$. Thus, $\operatorname{ord}\left(\mathcal{I}_{1}\right)=\operatorname{ord}\left(\mathcal{I}_{d}\right)=h+s-s_{i}$, and consequently, $\operatorname{ord}\left(g, u_{i 0}\right)=h+s-s_{i}$.

It remains to show that $\operatorname{ord}\left(g, u_{i j k}\right)\left(j=1, \ldots, n ; k=0,1, \ldots, s_{i}\right)$ and $\operatorname{ord}\left(g, u_{i \alpha}\right)$ cannot exceed $\operatorname{ord}\left(g, u_{i 0}\right)$. If $\operatorname{ord}\left(g, u_{i j k}\right)=l>\operatorname{ord}\left(g, u_{i 0}\right)$, then differentiating the identity $g\left(\mathbf{u} ; \zeta_{0}, \ldots, \zeta_{d}\right)=0$ w.r.t. $u_{i j k}^{(l)}$, we have $\frac{\partial g}{\partial u_{i j k}^{(l)}}\left(\mathbf{u} ; \zeta_{0}, \ldots, \zeta_{d}\right)=0$. Thus, $\frac{\partial g}{\partial u_{i j k}^{(l)}}$ can be divisible by $g$, a contradiction. So ord $\left(g, u_{i j k}\right) \leq \operatorname{ord}\left(g, u_{i 0}\right)$. Similarly, we can prove that $\operatorname{ord}\left(g, u_{i \alpha}\right) \leq \operatorname{ord}\left(g, u_{i 0}\right)$. Thus, $\operatorname{ord}\left(G, \mathbf{u}_{i}\right)=\operatorname{ord}\left(g, u_{i 0}\right)$.

In the following, we consider the factorization of the generalized Chow form. Denote $h+s-s_{i}$ by $h_{i}(i=0, \ldots, d)$, where $s=\sum_{l=0}^{d} s_{l}$. Now consider $G$ as a polynomial in $u_{00}^{\left(h_{0}\right)}$ with coefficients in $\mathcal{F}_{0}=\mathcal{F}\langle\widetilde{\mathbf{u}}\rangle\left(u_{00}, \ldots, u_{00}^{\left(h_{0}-1\right)}\right)$, where $\widetilde{\mathbf{u}}=\bigcup_{i=0}^{d} \mathbf{u}_{i} \backslash\left\{u_{00}\right\}$. Then, in an algebraic extension field of $\mathcal{F}_{0}$ we have

$$
g=A \prod_{\tau=1}^{t_{0}}\left(u_{00}^{\left(h_{0}\right)}-\gamma_{\tau}\right)
$$

where $t_{0}=\operatorname{deg}\left(g, u_{00}^{\left(h_{0}\right)}\right)$. Let $\xi_{\tau \rho k}=g_{\tau \rho k} / g_{\tau 0}\left(\rho=1, \ldots, n ; k=0, \ldots, s_{0}\right)$ and $\xi_{\tau \alpha}=g_{\tau \alpha} / g_{\tau 0}$, where $g_{\tau \rho k}=\left.\frac{\partial g}{\partial u_{0 \rho k}^{\left(h_{0}\right)}}\right|_{u_{00}^{\left(h_{0}\right)}=\gamma_{\tau}}, g_{\tau \alpha}=\left.\frac{\partial g}{\partial u_{0 \alpha}^{\left(h_{0}\right)}}\right|_{u_{00}^{\left(h_{0}\right)}=\gamma_{\tau}}$ and $g_{\tau 0}=$ $\left.\frac{\partial g}{\partial u_{00}^{\left(h_{0}\right)}}\right|_{u_{00}^{\left(h_{0}\right)}=\gamma_{\tau}}$. Similar to Section 4.4, we can uniquely define the derivatives of $\gamma_{\tau}$ and $\xi_{\tau \rho 0}$ to make them elements in a differential extension field of $\mathcal{F}\langle\widetilde{\mathbf{u}}\rangle$. From $g\left(\mathbf{u} ; \zeta_{0}, \ldots, \zeta_{d}\right)=0$, if we differentiate this equality w.r.t. $u_{0 \rho k}^{\left(h_{0}\right)}$, then we have

$$
\overline{\frac{\partial g}{\partial u_{0 \rho k}^{\left(h_{0}\right)}}}+\overline{\frac{\partial g}{\partial u_{00}^{\left(h_{0}\right)}}}\left(-\xi_{\rho}^{(k)}\right)=0,
$$

and if we differentiate $g\left(\mathbf{u} ; \zeta_{0}, \ldots, \zeta_{d}\right)=0$ w.r.t. $u_{0 \alpha}^{\left(h_{0}\right)}$, then

$$
\overline{\frac{\partial g}{\partial u_{0 \alpha}^{\left(h_{0}\right)}}}+\overline{\frac{\partial g}{\partial u_{00}^{\left(h_{0}\right)}}}\left(-\left(\xi^{\left(s_{0}\right)}\right)^{\alpha}\right)=0,
$$

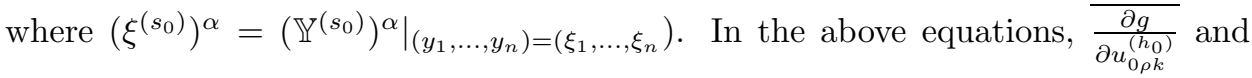
$\overline{\frac{\partial g}{\partial u_{0 \alpha}^{\left(h_{0}\right)}}}$ represent $\frac{\partial g}{\partial u_{0 \rho k}^{\left(h_{0}\right)}}$ and $\frac{\partial g}{\partial u_{0 \alpha}^{\left(h_{0}\right)}}$ when substituting $u_{i 0}$ by $\zeta_{i}$. For each $\rho=$ $1, \ldots, n$ and $k=0, \ldots, s_{0}$, multiplying the equations in (6.4) by $u_{0 \rho k}$, and for $\alpha \in \mathbb{Z}_{\geq 0}^{n\left(s_{0}+1\right)}, 1<|\alpha| \leq m_{0}$, multiplying the equations in 6.5. by $u_{0 \alpha}$ and then 
adding all of the equations obtained together, we have

$$
\zeta_{0} \overline{\frac{\partial g}{\partial u_{00}^{\left(h_{0}\right)}}}+\sum_{\rho=1}^{n} \sum_{k=0}^{s_{0}} u_{0 \rho k} \overline{\frac{\partial g}{\partial u_{0 \rho k}^{\left(h_{0}\right)}}}+\sum_{\substack{\alpha \in \mathbb{Z}_{\geq 0}^{n\left(s_{0}+1\right)} \\ 1<|\alpha| \leq m_{0}}} u_{0 \alpha} \overline{\frac{\partial g}{\partial u_{0 \alpha}^{\left(h_{0}\right)}}}=0 .
$$

Thus, the $\delta$-polynomial $u_{00} \frac{\partial g}{\partial u_{00}^{\left(h_{0}\right)}}+\sum_{\rho=1}^{n} \sum_{k=0}^{s_{0}} u_{0 \rho k} \frac{\partial g}{\partial u_{0 \rho k}^{\left(h_{0}\right)}}+\sum_{\substack{\alpha \in \mathbb{Z}_{\geq 0}^{n\left(s_{0}+1\right)} \\ 1<|\alpha| \leq m_{0}}} u_{0 \alpha} \frac{\partial g}{\partial u_{0 \alpha}^{\left(h_{0}\right)}} \quad$ vanishes at $\left(u_{00}, \ldots, u_{d 0}\right)=\left(\zeta_{0}, \ldots, \zeta_{d}\right)$. Since it is at most of the same order as $g$, it must be divisible by $g$. Also, since it has the same degree as $g$, there exists some $a \in \mathcal{F}$ such that

$$
u_{00} \frac{\partial g}{\partial u_{00}^{\left(h_{0}\right)}}+\sum_{\rho=1}^{n} \sum_{k=0}^{s_{0}} u_{0 \rho k} \frac{\partial g}{\partial u_{0 \rho k}^{\left(h_{0}\right)}}+\sum_{\substack{\alpha \in \mathbb{Z}_{\geq 0}^{n\left(s_{0}+1\right)} \\ 1<|\alpha| \leq m_{0}}} u_{0 \alpha} \frac{\partial g}{\partial u_{0 \alpha}^{\left(h_{0}\right)}}=a g .
$$

Setting $u_{00}^{\left(h_{0}\right)}=\gamma_{\tau}$ in both sides of the above equation, we have

$$
u_{00} g_{\tau 0}+\sum_{\rho=1}^{n} \sum_{k=0}^{s_{0}} u_{0 \rho k} g_{\tau \rho k}+\sum_{\substack{\alpha \in \mathbb{Z}_{\geq 0}^{n\left(s_{0}+1\right)} \\ 1<|\alpha| \leq m_{0}}} u_{0 \alpha} g_{\tau \alpha}=0
$$

or

$$
u_{00}+\sum_{\rho=1}^{n} \sum_{k=0}^{s_{0}} u_{0 \rho k} \xi_{\tau \rho k}+\sum_{\substack{\alpha \in \mathbb{Z}_{\geq 0}^{n\left(s_{0}+1\right)} \\ 1<|\alpha| \leq m_{0}}} u_{0 \alpha} \xi_{\tau \alpha}=0
$$

Then, we have

$$
\begin{gathered}
\left(u_{00}+\sum_{\rho=1}^{n} \sum_{k=0}^{s_{0}} u_{0 \rho k} \xi_{\tau \rho k}+\sum_{\substack{\alpha \in \mathbb{Z}_{\geq 0}^{n\left(s_{0}+1\right)} \\
1<|\alpha| \leq m_{0}}} u_{0 \alpha} \xi_{\tau \alpha}\right)^{\left(h_{0}\right)} \\
=\gamma_{\tau}+\left(\sum_{\rho=1}^{n} \sum_{k=0}^{s_{0}} u_{0 \rho k} \xi_{\tau \rho k}+\sum_{\substack{\alpha \in \mathbb{Z}_{\geq 0}^{n\left(s_{0}+1\right)} \\
1<|\alpha| \leq m_{0}}} u_{0 \alpha} \xi_{\tau \alpha}\right)^{\left(h_{0}\right)}=0 .
\end{gathered}
$$

We have the following theorem.

Theorem 6.4. Let $G\left(\mathbf{u}_{0}, \mathbf{u}_{1}, \ldots, \mathbf{u}_{d}\right)$ be the generalized Chow form of a $\delta$ - $\mathcal{F}$-variety of dimension $d$ and order $h$. Then, there exist $\xi_{\tau \rho}\left(\rho=1, \ldots, n ; \tau=1, \ldots, t_{0}\right)$ in a $\delta$-extension field of $\mathcal{F}\langle\widetilde{\mathbf{u}}\rangle$ such that

$$
G\left(\mathbf{u}_{0}, \mathbf{u}_{1}, \ldots, \mathbf{u}_{d}\right)=A\left(\mathbf{u}_{0}, \mathbf{u}_{1}, \ldots, \mathbf{u}_{d}\right) \prod_{\tau=1}^{t_{0}} \mathbb{P}_{0}\left(\xi_{\tau 1}, \ldots, \xi_{\tau n}\right)^{\left(h_{0}\right)}
$$

where $A\left(\mathbf{u}_{0}, \mathbf{u}_{1}, \ldots, \mathbf{u}_{d}\right)$ is in $\mathcal{F}\left\{\mathbf{u}_{0}, \mathbf{u}_{1}, \ldots, \mathbf{u}_{d}\right\}$ and $t_{0}=\operatorname{deg}\left(G, u_{00}^{\left(h_{0}\right)}\right)$. 
Proof. From what we have proved,

$$
\begin{aligned}
& G\left(\mathbf{u}_{0}, \mathbf{u}_{1}, \ldots, \mathbf{u}_{d}\right) \\
& \quad=A\left(\mathbf{u}_{0}, \mathbf{u}_{1}, \ldots, \mathbf{u}_{d}\right) \prod_{\tau=1}^{t_{0}}\left(u_{00}+\sum_{\rho=1}^{n} \sum_{k=0}^{s_{0}} u_{0 \rho k} \xi_{\tau \rho k}+\sum_{\substack{\alpha \in \mathbb{Z}_{\geq 0}^{n\left(s_{0}+1\right)} \\
1<|\alpha| \leq m_{0}}} u_{0 \alpha} \xi_{\tau \alpha}\right)^{\left(h_{0}\right)} .
\end{aligned}
$$

Denote $\xi_{\tau \rho 0}$ by $\xi_{\tau \rho}$. To complete the proof, it remains to show that $\xi_{\tau \rho k}=$ $\left(\xi_{\tau \rho 0}\right)^{(k)}\left(k=1, \ldots, s_{0}\right)$ and $\xi_{\tau \alpha}=\prod_{\rho=1}^{n} \prod_{j=0}^{s_{0}}\left(\left(\xi_{\tau \rho 0}\right)^{(j)}\right)^{\alpha_{\rho j}}$. From equation (6.4) and equation (6.5), we have $\xi_{\rho}^{(k)}=\frac{\frac{\rho=1}{\partial g}}{\partial u_{0 \rho k}^{\left(h_{0}\right)}} / \overline{\frac{\partial g}{\partial u_{00}^{\left(h_{0}\right)}}}\left(k=0, \ldots, s_{0}\right)$ and $\left(\xi^{\left(s_{0}\right)}\right)^{\alpha}=$ $\overline{\frac{\partial g}{\partial u_{0 \alpha}^{\left(h_{0}\right)}}} / \overline{\frac{\partial g}{\partial u_{00}^{\left(h_{0}\right)}}}$. So we have the equalities $\left(\overline{\frac{\partial g}{\partial u_{0 \rho 0}^{\left(h_{0}\right)}}} / \overline{\frac{\partial g}{\partial u_{00}^{\left(h_{0}\right)}}}\right)^{(k)}=\overline{\frac{\partial g}{\partial u_{0 \rho k}^{\left(h_{0}\right)}}} / \overline{\frac{\partial g}{\partial u_{00}^{\left(h_{0}\right)}}}$ and

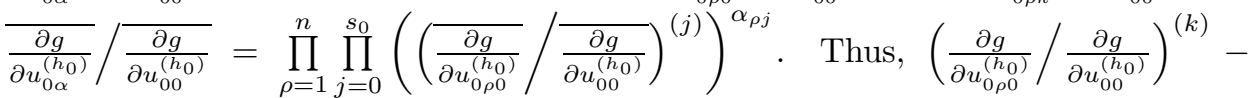
$\frac{\partial g}{\partial u_{0 \rho k}^{\left(h_{0}\right)}} / \frac{\partial g}{\partial u_{00}^{\left(h_{0}\right)}}$ and $\frac{\partial g}{\partial u_{0 \alpha}^{\left(h_{0}\right)}} / \frac{\partial g}{\partial u_{00}^{\left(h_{0}\right)}}-\prod_{\rho=1}^{n} \prod_{j=0}^{s_{0}}\left(\left(\frac{\partial g}{\partial u_{0 \rho 0}^{\left(h_{0}\right)}} / \frac{\partial g}{\partial u_{00}^{\left(h_{0}\right)}}\right)^{(j)}\right)^{\alpha_{\rho j}}$ vanish at $\left(u_{00}\right.$, $\left.\ldots, u_{d 0}\right)=\left(\zeta_{0}, \ldots, \zeta_{d}\right)$. Similar to the proof of Theorem 4.34, we can see that both of the differential polynomials $\left(\frac{\partial g}{\partial u_{0 \rho 0}^{\left(h_{0}\right)}} / \frac{\partial g}{\partial u_{00}^{\left(h_{0}\right)}}\right)^{(k)}-\frac{\partial g}{\partial u_{0 \rho k}^{\left(h_{0}\right)}} / \frac{\partial g}{\partial u_{00}^{\left(h_{0}\right)}}$ and $\frac{\partial g}{\partial u_{0 \alpha}^{\left(h_{0}\right)}} / \frac{\partial g}{\partial u_{00}^{\left(h_{0}\right)}}$ $-\prod_{\rho=1}^{n} \prod_{j=0}^{s_{0}}\left(\left(\frac{\partial g}{\partial u_{0 \rho 0}^{\left(h_{0}\right)}} / \frac{\partial g}{\partial u_{00}^{\left(h_{0}\right)}}\right)^{(j)}\right)^{\alpha_{\rho j}}$ vanish at $u_{00}^{\left(h_{0}+j\right)}=\gamma_{\tau}^{(j)}(j \geq 0)$. Thus, $\xi_{\tau \rho 0}^{(k)}=$ $\xi_{\tau \rho k}$ and $\xi_{\tau \alpha}-\prod_{\rho=1}^{n} \prod_{j=0}^{s_{0}}\left(\left(\xi_{\tau \rho 0}\right)^{(j)}\right)^{\alpha_{\rho j}}=0$. The proof is completed.

Theorem 6.5. The points $\left(\xi_{\tau 1}, \ldots, \xi_{\tau n}\right)\left(\tau=1, \ldots, t_{0}\right)$ in (6.6) are generic points of the $\delta$ - $\mathcal{F}$-variety $V$ and satisfy the equations

$$
\mathbb{P}_{\sigma}\left(y_{1}, \ldots, y_{n}\right)=u_{\sigma 0}+\sum_{\rho=1}^{n} \sum_{k=0}^{s_{\sigma}} u_{\sigma \rho k} y_{\rho}^{(k)}+f_{\sigma}=0 \quad(\sigma=1, \ldots, d) .
$$

Proof. The proof is similar to that of Theorem 4.34

Theorem 6.6. Let $G\left(\mathbf{u}_{0}, \ldots, \mathbf{u}_{d}\right)$ be the generalized Chow form of $V$ and $S_{G}=$ $\frac{\partial G}{\partial u_{00}^{(h)}}$ with $\operatorname{ord}\left(G, u_{00}\right)=h_{0}$. Suppose that $\mathbf{u}_{i}(i=0, \ldots, d)$ are $\delta$-specialized to sets $\mathbf{v}_{i}$ of specific elements in $\mathcal{E}$ and $\overline{\mathbb{P}}_{i}(i=0, \ldots, d)$ are obtained by substituting $\mathbf{u}_{i}$ by $\mathbf{v}_{i}$ in $\mathbb{P}_{i}$. If $\overline{\mathbb{P}}_{i}=0(i=0, \ldots, d)$ meet $V$, then $G\left(\mathbf{v}_{0}, \ldots, \mathbf{v}_{d}\right)=0$. Furthermore, if $G\left(\mathbf{v}_{0}, \ldots, \mathbf{v}_{d}\right)=0$ and $S_{G}\left(\mathbf{v}_{0}, \ldots, \mathbf{v}_{d}\right) \neq 0$, then the $d+1 \delta$-hypersurfaces $\overline{\mathbb{P}}_{i}=0$ $(i=0, \ldots, d)$ meet $V$.

Proof. The proof is similar to that of Theorem 4.43

6.2. Differential resultant of multivariate differential polynomials. As an application of the generalized Chow form, we can define the differential resultant of $n+1$ generic $\delta$-polynomials in $n$ variables. Let $\mathcal{I}=[0]$ be the $\delta$-ideal generated by 0 in $\mathcal{F}\{\mathbb{Y}\}$. Then $\operatorname{dim}(\mathcal{I})=n$. Let $G\left(\mathbf{u}_{0}, \mathbf{u}_{1}, \ldots, \mathbf{u}_{n}\right)$ be the generalized Chow form for $\mathcal{I}$. Then we will define $G\left(\mathbf{u}_{0}, \mathbf{u}_{1}, \ldots, \mathbf{u}_{n}\right)$ to be the differential resultant for the $n+1$ generic $\delta$-polynomials given in (6.1). 
Definition 6.7. The differential resultant for the $n+1$ generic $\delta$-polynomials $\mathbb{P}_{i}$ in (6.1) is defined to be the generalized Chow form of $\mathcal{I}=[0]$ associated with these $\mathbb{P}_{i}$, and will be denoted by $R\left(\mathbf{u}_{0}, \ldots, \mathbf{u}_{n}\right)=G\left(\mathbf{u}_{0}, \ldots, \mathbf{u}_{n}\right)$.

Theorem 6.8. Let $R\left(\mathbf{u}_{0}, \ldots, \mathbf{u}_{n}\right)$ be the differential resultant of the $n+1 \delta$ polynomials $\mathbb{P}_{0}, \ldots, \mathbb{P}_{n}$ given in (6.1) with $\operatorname{ord}\left(\mathbb{P}_{i}\right)=s_{i}$ and $\operatorname{deg}\left(\mathbb{P}_{i}\right)=m_{i}$, where $\mathbf{u}_{i}=\left(u_{i 0}, \ldots, u_{i j k}, \ldots, u_{i \alpha}, \ldots\right)(i=0, \ldots, n)$. Denote $s=\sum_{i=0}^{n} s_{i}, D=\max _{i=0}^{n}\left\{m_{i}\right\}$ and $\mathbf{u}=\bigcup_{i=0}^{n} \mathbf{u}_{i} \backslash\left\{u_{i 0}\right\}$. Then there exist $h_{j k} \in \mathcal{F}\langle\mathbf{u}\rangle\left[y_{1}, \ldots, y_{n}, \ldots, y_{1}^{(s)}, \ldots, y_{n}^{(s)}\right]$ such that

$$
R\left(\mathbf{u}_{0}, \ldots, \mathbf{u}_{n}\right)=\sum_{j=0}^{n} \sum_{k=0}^{s-s_{j}} h_{j k} \delta^{k} \mathbb{P}_{j} .
$$

Moreover, the degree of $h_{j k}$ in $\mathbb{Y}$ is bounded by $(s n+n)^{2} D^{s n+n}+D(s n+n)$.

Proof. Let $\mathcal{J}$ be the $\delta$-ideal generated by $\mathbb{P}_{0}, \ldots, \mathbb{P}_{n}$ in $\mathcal{F}\left\{\mathbf{u}_{0}, \ldots, \mathbf{u}_{n}, y_{1}, \ldots, y_{n}\right\}$. Let $\mathscr{R}$ be the elimination ranking $\mathbf{u} \prec y_{n} \prec \cdots \prec y_{1} \prec u_{n 0} \prec \cdots \prec u_{00}$ with arbitrary ranking endowed on $\Theta(\mathbf{u})=(\theta u: u \in \mathbf{u} ; \theta \in \Theta)$. Clearly, $\mathcal{J}$ is a prime $\delta$-ideal with $\mathbb{P}_{0}, \ldots, \mathbb{P}_{n}$ as its characteristic set w.r.t. $\mathscr{R}$. Thus, $\mathbf{u} \cup\left\{y_{1}, \ldots, y_{n}\right\}$ is a parametric set of $\mathcal{J}$. From the definition of $R, R \in \mathcal{J}$. In $R\left(\mathbf{u}_{0}, \ldots, \mathbf{u}_{n}\right)=$ $G\left(\mathbf{u}_{0}, \ldots, \mathbf{u}_{n}\right)=g\left(\mathbf{u} ; u_{00}, \ldots, u_{n 0}\right)$, let $u_{i 0}(i=0, \ldots, n)$ be replaced respectively by

$$
\mathbb{P}_{i}-\sum_{j=1}^{n} \sum_{k=0}^{s_{i}} u_{i j k} y_{j}^{(k)}-\sum_{\substack{\alpha \in \mathbb{Z}_{\geq 0}^{n\left(s_{i}+1\right)} \\ 1<|\alpha| \leq m_{i}}} u_{i \alpha}\left(\mathbb{Y}^{\left(s_{i}\right)}\right)^{\alpha}, \quad(i=0, \ldots, d),
$$

and let $R$ be expanded as a polynomial in $\mathbb{P}_{0}, \ldots, \mathbb{P}_{n}$ and their derivatives. The term not involving $\mathbb{P}_{0}, \ldots, \mathbb{P}_{n}$ or their derivatives will be a $\delta$-polynomial only involving $\mathbf{u} \cup$ $\left\{y_{1}, \ldots, y_{n}\right\}$ which also belong to $\mathcal{J}$. Since $\mathcal{J} \cap \mathcal{F}\left\{\mathbf{u}, y_{1}, \ldots, y_{n}\right\}=\{0\}$, such a term will be identically zero. So $R$ is a linear combination of $\mathbb{P}_{0}, \ldots, \mathbb{P}_{n}$ and some of their derivatives. Since ord $\left(R, u_{i 0}\right)=s-s_{i}$, the above expansion for $R$ involves $\mathbb{P}_{i}$ only up to the order $s-s_{i}$ and the coefficients in the linear combination are $\delta$-polynomials in $\mathcal{F}\{\mathbf{u}\}\left[y_{1}, \ldots, y_{n}, \ldots, y_{1}^{(s)}, \ldots, y_{n}^{(s)}\right]$. Denote $\mathcal{R}=\mathcal{F}\langle\mathbf{u}\rangle\left[y_{1}, \ldots, y_{n}, \ldots, y_{1}^{(s)}, \ldots, y_{n}^{(s)}\right]$. Thus, $R \in\left(\delta^{s-s_{0}} \mathbb{P}_{0}, \ldots, \delta \mathbb{P}_{0}, \mathbb{P}_{0}, \ldots, \delta^{s-s_{n}} \mathbb{P}_{n}, \ldots, \delta \mathbb{P}_{n}, \mathbb{P}_{n}\right) \subseteq \mathcal{R}$, which implies that $\left(\delta^{s-s_{0}} \mathbb{P}_{0}, \ldots, \delta \mathbb{P}_{0}, \mathbb{P}_{0}, \ldots, \delta^{s-s_{n}} \mathbb{P}_{n}, \ldots, \delta \mathbb{P}_{n}, \mathbb{P}_{n}\right)$ in $\mathcal{R}$ is the unit ideal. By [31, Theorem 1], there exist $A_{j k} \in \mathcal{R}$ with $\operatorname{deg}\left(A_{j k}\right) \leq(s n+n)^{2} D^{s n+n}+D(s n+n)$ such that

$$
1=\sum_{j=0}^{n} \sum_{k=0}^{s-s_{j}} A_{j k} \delta^{k} \mathbb{P}_{j}
$$

where $D=\max \left\{m_{0}, m_{1}, \ldots, m_{n}\right\}$. If we multiply the above equation by $R$ and denote $A_{j k} R$ by $h_{j k}$, we complete the proof.

As a consequence of the above five theorems proved in this section, the properties of the differential resultant listed in Theorem 1.3 are proved.

Let $R\left(\mathbf{u}_{0}, \ldots, \mathbf{u}_{n}\right)$ be the differential resultant for the $n+1$ generic $\delta$-polynomials $\mathbb{P}_{i}$ in (6.1). When each $\mathbf{u}_{i}$ are specialized to specific elements $\mathbf{v}_{i} \in \mathcal{F}^{n+1}, R\left(\mathbf{v}_{0}, \ldots\right.$, $\left.\mathbf{v}_{n}\right)$ is called the differential resultant of $\overline{\mathbb{P}}_{i}(i=0, \ldots, n)$ which are obtained by replacing $\mathbf{u}_{i}$ by $\mathbf{v}_{i}$ in $\mathbb{P}_{i}$. By Theorem 6.8, the vanishing of the differential resultant for $n+1 \delta$-polynomials in $\mathcal{F}\{\mathbb{Y}\}$ is a necessary condition for them to have a common solution. 
Remark 6.9. It is easy to see that if $s_{i}=0$, then the differential resultant of $\mathbb{P}_{i}(i=$ $0, \ldots, n)$ becomes the Macaulay resultant for $n+1$ polynomials in $n$ variables $[9,18$. From Theorem 1.3, we see that the differential resultant has similar properties to that of the Macaulay resultant. Special attention should be payed to the second property which is a differential analog to the so-called Poisson type formulas for algebraic resultants 28. Also note that many properties of the Macaulay resultant are yet to be extended to the differential case. The most significant one might be to find a matrix representation for the differential resultant similar to the one given in [9, p.102]. Note that such a formula was claimed to be given in [5, 6, which is not correct, as we mentioned in Section 1 of this paper. As a latest development, we defined the differential sparse resultant and proposed a single exponential algorithm to compute it [26].

Similar to the differential Chow form, the differential resultant can be computed with the differential elimination algorithms [1, 7, 32, 39, 14.

Example 6.10. The simplest nonlinear differential resultant is the case $n=1, d_{0}=$ $d_{1}=2, s_{0}=0, s_{1}=1$. Denote $y_{1}$ by $y$. Let $\mathbb{P}_{0}=u_{00}+u_{01} y+u_{02} y^{2}, \mathbb{P}_{1}=u_{10}+$ $u_{11} y+u_{12} y^{\prime}+u_{13} y^{2}+u_{14} y y^{\prime}+u_{15}\left(y^{\prime}\right)^{2}$. Then the differential resultant for $\mathbb{P}_{0}$ and $\mathbb{P}_{1}$ is a $\delta$-polynomial $R\left(\mathbf{u}_{0}, \mathbf{u}_{1}\right)$ such that $\operatorname{ord}\left(R, \mathbf{u}_{0}\right)=1, \operatorname{ord}\left(R, \mathbf{u}_{1}\right)=0$ and $R$ is $\delta$-homogenous of degree 8 in $\mathbf{u}_{0}$ and degree 2 in $\mathbf{u}_{1}$, respectively. Totally, $R$ has 206 terms. Moreover, $R$ has a matrix representation which is a factor of the determinant of the coefficient matrix of $\mathbb{P}_{0}, y^{\prime} \mathbb{P}_{0}, y^{2} \mathbb{P}_{0}, y y^{\prime} \mathbb{P}_{0}, y^{\prime 2} \mathbb{P}_{0}, \mathbb{P}_{0}^{\prime}, y \mathbb{P}_{0}^{\prime}, y^{\prime} \mathbb{P}_{0}^{\prime}, y y^{\prime} \mathbb{P}_{0}^{\prime}, y^{\prime 2} \mathbb{P}_{0}, \mathbb{P}_{1}$, $y \mathbb{P}_{1}, y^{\prime} \mathbb{P}_{0}, y y^{\prime} \mathbb{P}_{1}$ w.r.t. the monomials $\left\{y^{l_{0}}\left(y^{\prime}\right)^{l_{1}} \mid 0 \leq l_{0} \leq 4,0 \leq l_{1}<4, l_{0}+l_{1} \leq 4\right\}$.

\section{Conclusion}

In this paper, an intersection theory for generic differential polynomials is presented by giving the explicit formulas for the dimension and order of the intersection of an irreducible differential variety with a generic differential hypersurface. As a consequence, we show that the differential dimension conjecture is true for generic differential polynomials.

The Chow form for an irreducible differential variety is defined. Most of the properties of the algebraic Chow form have been extended to its differential counterpart. In particular, we introduce the concept of differential Chow quasi-variety for a special class of differential algebraic cycles. Furthermore, the generalized Chow form for an irreducible differential variety is also defined and its properties are proved. As an application of the generalized differential Chow form, we can give a rigorous definition for the differential resultant and establish its properties which are similar to that of the Sylvester resultant of two univariate polynomials and the Macaulay resultant of multivariate polynomials.

The results given in this paper enrich the field of differential algebraic geometry. Further, many new problems can be raised naturally. Some of them are already mentioned in Remarks 4.44 and 6.9. We mentioned in Section 1 that the algebraic Chow form has many important applications. It is very interesting to see whether some of these applications can be extended to the differential case.

As we mentioned in Section 5, the theory of differential Chow quasi-varieties is not fully developed, and the main difficulty is to develop an elimination theory for mixed systems with both algebraic and differential equations.

In this paper, we only consider Chow forms for affine differential varieties. It is not difficult to extend most of the results in this paper to the differential Chow form 
of differentially projective varieties. Note that differentially projective varieties were defined by Kolchin in [24]. It is expected that Theorems 4.43, 4.45, and 5.7 could be improved for differentially projective varieties.

\section{REFERENCES}

[1] F. Boulier, F. Lemaire, and M.M. Maza. Computing Differential Characteristic Sets by Change of Ordering. Journal of Symbolic Computation, 45(1), 124-149, 2010. MR2568902 (2010k:13042)

[2] J. B. Bost, H. Gillet, and C. Soulé. Heights of Projective Varieties and Postive Green Forms. Journal of Amer. Math. Soc., 7(4), 903-1027, 1994. MR 1260106 (95j:14025)

[3] W. D. Brownawell. Bounds for the Degrees in the Nullstellensatz. The Annals of Mathematics, 126(3), 577-591, 1987. MR916719 (89b:12001)

[4] A. Buium and P.J. Cassidy. Differential Algebraic Geometry and Differential Algebraic Groups. In H. Bass et al. eds, Selected Works of Ellis Kolchin, with Commentary, 567-636, American Mathematical Society, Providence, RI, 1998. MR.1677530 (2000g:01042)

[5] G. Carra'-Ferro. A Resultant Theory for the Systems of Two Ordinary Algebraic Differential Equations. Appl. Algebra Engrg. Comm. Comput., 8, 539-560, 1997. MR1486378 (99c:13046)

[6] G. Carra'-Ferro. A Resultant Theory for Ordinary Algebraic Differential Equations. Lecture Notes in Computer Science, 1255, 55-65, Springer, 1997. MR.1634105 (99m:12008)

[7] S. C. Chou and X. S. Gao. Automated Reasoning in Differential Geometry and Mechanics: Part I. An Improved Version of Ritt-Wu's Decomposition Algorithm. Journal of Automated Reasoning, 10, 161-172, 1993. MR1215347(94h:03022)

[8] R. M. Cohn. Order and Dimension. Proc. Amer. Math. Soc., 87(1), $1983 . \quad$ MR677218 (83m:12038)

[9] D. Cox, J. Little, D. O'Shea. Using Algeraic Geometry. Springer, 1998. MR.1639811 (99h:13033)

[10] T. Cluzeau and E. Hubert. Rosolvent Representation for Regular Differential Ideals. Appl. Algebra Engrg. Comm. Comput., 13, 395-425, 2003. MR1959171 (2004e:12005)

[11] D. Eisenbud, F. O. Schreyer, and J. Weyman. Resultants and Chow Forms via Exterior Syzygies. Journal of Amer. Math. Soc., 16(3), 537-579, 2004. MR.1969204 (2004j:14067)

[12] X. S. Gao and S. C. Chou. A Zero Structure Theorem for Differential Parametric Systems. Journal of Symbolic Computation, 16, 585-595, 1994. MR.1279535(96d:12008)

[13] I. M. Gel'fand, M. Kapranov, and A. Zelevinsky. Discriminants, Resultants and Multidimensional Determinants. Birkhäuser, Boston, 1994. MR1264417 (95e:14045)

[14] O. Golubitsky, M. Kondratieva, and A. Ovchinnikov. Algebraic Transformation of Differential Characteristic Decompositions from One Ranking to Another. Journal of Symbolic Computation, 44, 333-357, 2009. MR2494979(2010e:12009)

[15] W.V.D. Hodge and D. Pedoe. Methods of Algebraic Geometry, Volume I. Cambridge University Press, 1968.

[16] W.V.D. Hodge and D. Pedoe. Methods of Algebraic Geometry, Volume II. Cambridge University Press, 1968.

[17] G. Jeronimo, T. Krick, J. Sabia, and M. Sombra. The Computational Complexity of the Chow Form. Foundations of Computational Mathematics, 4(1), 41-117, 2004. MR2035410 (2005c:14083)

[18] J. P. Jouanolou. Le Formalisme du Rèsultant. Advances in Mathematics, 90(2), 117-263, 1991. MR:1142904 (93g:14003)

[19] E. R. Kolchin. Differential Algebra and Algebraic Groups. Academic Press, New York and London, 1973. MR0568864 (58:27929)

[20] E. R. Kolchin. Extensions of Differential Fields, I. Annals of Mathematics, 43 , 724-729, 1942. MR0007011(4:72b)

[21] E. R. Kolchin. Extensions of Differential Fields, III. Bull. Amer. Math. Soc., 53 , 397-401, 1947. MR0019590(8:432i)

[22] E. R. Kolchin. The Notion of Dimension in the Theory of Algebraic Differential Equations. Bull. Amer. Math. Soc., 70, 570-573, 1964. MR0168555(29:5816)

[23] E. R. Kolchin. A Problem on Differential Polynomials. Contemporary Mathematics, 131, 449-462, 1992. MR1175849 (94a:12013) 
[24] E. R. Kolchin. Differential Equations in a Projective Space and Linear Dependence over a Projective Variety. In Contributions to Analysis: A Colletion of Papers Dedicated to Lipman Bers, Academic Press, 195-214, 1974. MR0352067 (50:4555)

[25] M.V. Kondratieva, A.B. Levin, A.V. Mikhalev, E.V. Pankratiev. Differential and Difference Dimension Polynomials. Kluwer Academic Publishers, 1999. MR.1676955 (2001c:12006)

[26] W. Li, X. S. Gao, C. M. Yuan. Sparse Differential Resultan. ISSAC 2011 - Proceedings of the 36th International Symposium on Symbolic and Algebraic Computation, 225-232, ACM, New York, 2011. MR.2895216

[27] Yu. V. Nesterenko. Estimates for the Orders of Zeros of Functions of a Certain Class and Applications in the Theory of Transcendental Numbers. Izv. Akad. Nauk SSSR Ser. Mat. 41, 253-284, 1977. MR0491535(58:10774)

[28] P. Pedersen and B. Sturmfels. Product Formulas for Resultants and Chow Forms. Mathematische Zeitschrift, 214(1), 377-396, 1993. MR1245200 (94m:14068)

[29] P. Philippon. Critères pour L'independance Algebrique. Inst. Hautes Ètudes Sci. Publ. Math., 64, 5-52, 1986. MR876159 (88h:11048)

[30] J. Plumer. Resultants and the Algebraicity of the Join Pairing on Chow Varieties. Trans. Amer. Math. Soc., 349(6), 2187-2209, 1997. MR1407499(98e:14005)

[31] J. F. Ritt. Differential Equations from the Algebraic Standpoint. Amer. Math. Soc., New York, 1932.

[32] J. F. Ritt. Differential Algebra. Amer. Math. Soc., New York, 1950. MR0035763 (12:7c)

[33] F. Rouillier. Solving Zero-dimensional Polynomial Systems through Rational Univariate Representation. Appl. Algebra Engrg. Comm. Comput., 9, 433-461, 1999. MR1697179 (2000e:13038)

[34] B. Sadik. A Bound for the Order of Characteristic Set Elements of an Ordinary Prime Differential Ideal and some Applications. Appl. Algebra Engrg. Comm. Comput., 10, 251-268, 2000. MR1751434 (2001f:12009)

[35] A. Rosenfeld. Specializations in Differential Algebra. Trans. Amer. Math. Soc., 90(3), 394407, 1959. MR0107642(21:6367)

[36] S. L. Rueda and J. R. Sendra. Linear Complete Differential Resultants and the Implicitization of Linear DPPEs. Journal of Symbolic Computation, 45(3), 324-341, 2010. MR2578342 (2011b:12011)

[37] A. Seidenberg. An Elimination Theory for Differential Algebra. Univ. California Publication in Math., 3, 31-65, 1956. MR0082487 (18:558a)

[38] A. Seidenberg. On the Chow Form. Math. Ann. 212, 183-190, 1975. MR0376667 (51:12842)

[39] W. Y. Sit. The Ritt-Kolchin Theory for Differential Polynomials. In Differential Algebra and Related Topics, 1-70, World Scientific, 2002. MR1921694(2003g:12010)

[40] B. Sturmfels. Sparse Elimination Theory. In Eisenbud, D., Robbiano, L. eds., Computational Algebraic Geometry and Commutative Algebra. 264-298, Cambridge University Press, 1993. MR 1253995 (94k:13035)

[41] B. L. van der Waerden. Einführung in die Algebraische Geometrie. Teubner, Berlin, 1939.

[42] W. T. Wu. Basic Principles of Mechanical Theorem Proving in Elementary Geometries. Journal of Automated Reasoning, 4(3), 207-235, 1984. MR0795000 (87g:03017)

[43] W. T. Wu. Mathematics Mechanization. Science Press/Kluwer, Beijing, 2000. MR.1834540 (2003a:01005)

KLmm, Academy of Mathematics and Systems Science, Chinese Academy of Sciences, Beijing 100190, People's Republic of China

E-mail address: xgao@mmrc.iss.ac.cn

KLMM, Academy of Mathematics and Systems Science, Chinese Academy of Sciences, Beijing 100190, People's Republic of China

E-mail address: liwei@mmc.iss.ac.cn

KLmm, Academy of Mathematics and Systems Science, Chinese Academy of Sciences, Beijing 100190, People's Republic of China

E-mail address: cmyuan@mmrc.iss.ac.cn 